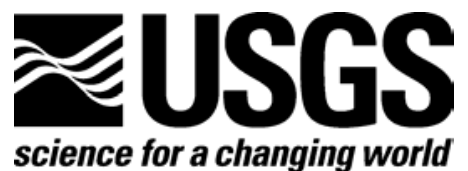

In Cooperation with the Southern Nevada Water Authority (SNWA)

\title{
Audiomagnetotelluric data from Spring, Cave, and Coyote Spring Valleys, Nevada
}

By Darcy K. McPhee, Bruce A. Chuchel, and Louise Pellerin

Open-File Report 2006-1164

U.S. Department of the Interior

U.S. Geological Survey 


\section{Contents}

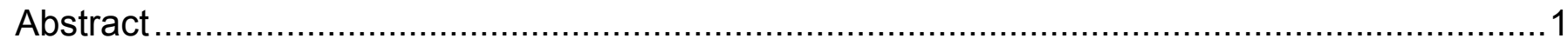

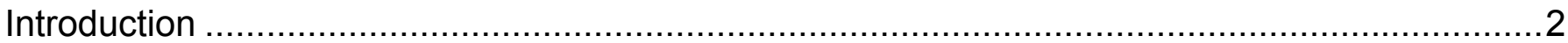

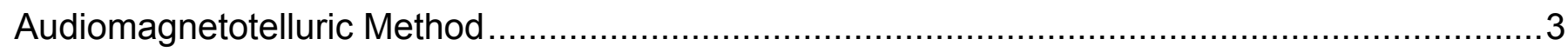

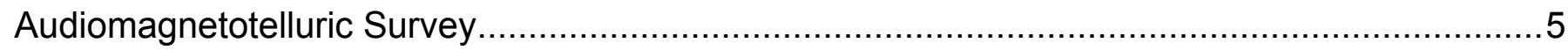

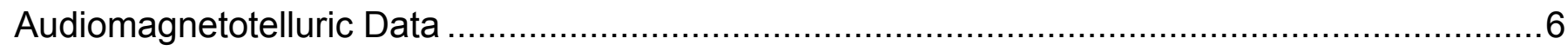

Acknowledgments ……

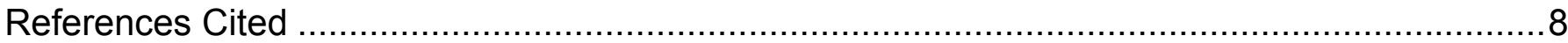

\section{Figures}

1. Topographic maps of study area showing locations of $A M T$ profiles $A, B$, and $E(A)$ and profile $C(B)$.

\section{Tables}

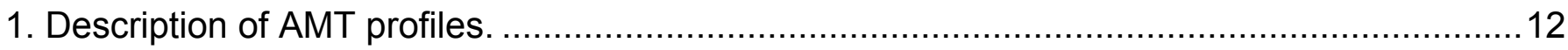

2. Sounding numbers, locations and elevations of stations along four AMT profiles .................12

\section{Appendix}

A. Sounding curves 


\title{
Audiomagnetotelluric data from Spring, Cave, and Coyote Spring Valleys, Nevada
}

\author{
By Darcy K. McPhee ${ }^{1}$, Bruce A. Chuchel ${ }^{1}$, and Louise Pellerin ${ }^{2}$
}

\section{Abstract}

Audiomagnetotelluric (AMT) data along four profiles in Spring, Cave, and Coyote Spring Valleys are presented here. The AMT method is used to estimate the electrical resistivity of the earth over depth ranges of a few meters to greater than one kilometer. This method is a valuable tool for revealing subsurface structure and stratigraphy within the Basin and Range of eastern Nevada, therefore helping to define the geohydrologic framework in this region. We collected AMT data using the Geometrics StrataGem EH4 system, a four-channel, natural and controlledsource tensor system recording in the range of 10 to $92,000 \mathrm{~Hz}$. To augment the low signal in the natural field, an unpolarized transmitter comprised of two horizontal-magnetic dipoles was used from 1,000 to 70,000 Hz. Profiles were $1.4-12.6 \mathrm{~km}$ in length with station spacing of 100-400 $\mathrm{m}$. Data were recorded with the electrical (E) field parallel to and perpendicular to the regional geologic strike direction. Station locations and sounding curves, showing apparent resistivity, phase data, and coherency data, are presented here.

\footnotetext{
${ }^{1}$ U.S. Geological Survey, 345 Middlefield Rd., MS 989, Menlo Park, CA 94025

${ }^{2}$ Green Engineering, Inc., 2215 Curtis St., Berkeley, CA 94702
} 


\section{Introduction}

The Basin and Range province is an arid, mountainous, sparsely populated region of the western United States. Here, ground water is organized into extensive regional systems (Harrill and Prudic, 1998) where it can flow between adjacent topographic ranges and basins. Much of the structure that controls the hydrogeology of the valleys in eastern Nevada is obscured by sediments, hence geophysical investigations are underway to characterize the subsurface structures and stratigraphy influencing ground-water resources. The gravity method has been used to estimate the structure and depth of the basins in eastern Nevada (Mankinen et al., 2006; Scheirer, 2005), however densities of silicic ash-flow tuff units in the area may be comparable to the average density of basin sediment-fill causing difficulty in resolving the subsurface geometry between the basin-fill and volcanic rocks using the gravity method alone.

In this study, the audiomagnetotelluric (AMT) method was tested in Spring, Cave, and Coyote Spring valleys (Figure 1) to see if it is a feasible approach for mapping geologic/tectonic structures and thus could contribute significantly to the regional hydrological model in a typical Basin and Range setting. During the fall of 2004 and summer of 2005 AMT data were acquired along four profile lines (Figure 1). The AMT data obtained in this study were acquired to delineate range-front faults, structure, and stratigraphy within the basins as well as the geometry of the basins as a framework for hydrogeological modeling.

The purpose of this report is to release the AMT sounding data. No interpretation of the data is included. 


\section{Audiomagnetotelluric Method}

The magnetotelluric (MT) method is a geophysical technique, which uses the earth's natural electromagnetic (EM) fields as a source to investigate the electrical resistivity structure of the subsurface (Vozoff, 1991). The resistivity of geologic units is largely dependent upon their fluid content, porosity, degree of fracturing, temperature, and conductive mineral content (Keller, 1989). Saline fluids within the pore spaces and fracture openings can reduce resistivities in a rock matrix. Resistivity can be lowered by the presence of conductive clay minerals, carbon, and metallic mineralization. Increased temperatures cause higher ionic mobility and mineral activation energy, reducing rock resistivities significantly. Unaltered, unfractured igneous rocks are normally very resistive, with values typically 1,000 ohm-m or greater. Fault zones can appear as low resistivity units of less than $100 \mathrm{ohm}-\mathrm{m}$ when they are comprised of rocks fractured enough to have hosted fluid transport and consequent mineralogical alteration (Eberhart-Phillips and others, 1995).

Carbonate rocks are moderately to highly resistive, with values of hundreds to thousands of ohm-m depending upon their fluid content, porosity, fracturing, and impurities. Marine shale, mudstone, and clay-rich alluvium are normally very conductive - a few ohm-m to tens of ohm-m. Unaltered, metamorphic, non-graphitic rocks are moderately to highly resistive. Tables of electrical resistivity for a variety of rocks, minerals and geological environments may be found in Keller (1987) and Palacky (1987).

Using the same theory, the audiomagnetotelluric (AMT) method is used to estimate the electrical resistivity of the Earth over depth ranges of a few meters to greater than one kilometer (Zonge and Hughes, 1991). The Geometrics StrataGem EH-4 system, used in this study, utilizes both natural and man-made electromagnetic signals to obtain a continuous electrical sounding of the Earth beneath the measurement site (Geometrics, 2000). AMT systems are used to estimate the 
electrical impedance at the Earth's surface from a series of simultaneous measurements of local electrical (E) and magnetic $(\mathrm{H})$ field fluctuations made over a period of several minutes. The surface impedance is a complex function of frequency where higher-frequency data are influenced by shallow or nearby features, and lower-frequency data are influenced by structures at greater depth and distance. An AMT sounding provides an estimate of vertical resistivity beneath the receiver site and also indicates the geoelectric complexity at the sounding site. In areas where the resistivity distribution does not change rapidly from station to station, the resistivity sounding is a reasonable estimate of the geoelectric layering beneath the site.

The Stratagem system consists of two basic components; a receiver and a transmitter (Geometrics, 2000). The natural source is worldwide lightning, but in the frequency range above $1,000 \mathrm{~Hz}$, natural signals are typically weak and a transmitter is used to augment the natural field and improve data quality. The unpolarized transmitter is comprised of two horizontal-magnetic dipoles and transmits signals from 1,000 to 70,000 Hz. The battery-powered system is compact and portable. Data are recorded in the range of 92,000 to $10 \mathrm{~Hz}$. Surface impedance results are immediately displayed as a resistivity sounding. Data are subsequently modeled using twodimensional inversion algorithms.

The natural electric and magnetic fields are measured in two orthogonal, horizontal directions. The tensor impedance, parameterized as apparent resistivity and phase, are obtained from the time-series signals. Signals are converted to complex cross-spectra using a Fouriertransform technique. A least-squares, cross-spectral analysis (Geometrics, 2000) is then used to solve for a transfer function (impedance) that relates the observed electric fields to the magnetic fields under the assumption that the earth consists of a two-input, two-output, linear system with the magnetic fields as input and the electric fields as output. 
For a two-dimensional (2-D) earth, the diagonal terms of the impedance tensor are zero. The off-diagonal terms are decoupled into transverse electric (TE) and transverse magnetic (TM) modes. When the geology satisfies the 2-D assumption, the MT and AMT data for the TE mode measures electric field parallel to geologic strike, and the data for the TM mode measures electric field perpendicular to geologic strike. Data are processed with a fixed rotation parallel and perpendicular to regional strike. The MT and AMT methods are well suited for studying complicated geological environments because the electric and magnetic relations are sensitive to vertical and horizontal variations in resistivity. The method is capable of establishing whether the EM fields are responding to subsurface terranes of 1-, 2-, or 3-dimensions. For more information on this technique, see Telford and others (1991) for an introduction to the MT method and Vozoff (1991) for a more advanced treatment.

\section{Audiomagnetotelluric Survey}

AMT sounding data were acquired along four profile lines in eastern Nevada (Figure 1). A description of each line is given in Table 1. In addition to these profiles, a fifth transect, Profile D, was attempted in Coyote Spring Valley (Figure 1). However, this line was aborted due to the distortion of the data from nearby power lines, and the data are not presented here. Access to all but a few stations was with a truck along dirt roads. All soundings were collected with a Geometrics StrataGem EH-4 system (Geometrics, 2000). Horizontal electric fields were measured using Model BE-26 Buffered Active High Frequency dipoles and stainless steel electrodes in an X-shaped array with nominal dipole lengths of $40 \mathrm{~m}$. The two, horizontal and orthogonal components of the magnetic field are measured in the same direction as the electric-field array with permalloy-cored 
BF-10 induction coils. Data were acquired in two frequency bands: 92,000 to $800 \mathrm{~Hz}$ and 1000 to $10 \mathrm{~Hz}$.

Through experimentation we found that the optimal transmitter distance, at which the measurement site is located in the transmitter's far field and yet receives adequate signal power, was 200 to $300 \mathrm{~m}$ from the receiver array, and we were able to acquire two stations for each transmitter location.

Table 2 shows the AMT station locations and elevations for lines A, B, C, and E. Locations and elevations were recorded using a Garmin global positioning system during field acquisition.

\section{Audiomagnetotelluric Data}

After transforming the recorded time-series data to the frequency domain, standard processing (Geometrics, 2000) was employed to determine apparent resistivity and phase tensor at each site. Apparent resistivity is the ratio of one component of the electric field magnitude over the orthogonal component of the magnetic field magnitude, normalized by the frequency and the magnetic susceptibility for free space. The impedance phase is the arctangent of the unnormalized ratio. The apparent resistivity and phase are related through a Hilbert transform; the phase is proportional to the slope of the apparent resistivity curve on a log-log plot, but from a baseline of 45 degrees (Vozoff, 1991).

Predicted values of the electric field can be computed from the measured values of the magnetic field (Vozoff, 1991). The coherence of the predicted electric field with the measured electric field is a measure of the signal-to-noise ratio provided in the E-predicted coherency plots. 
Values are normalized between 0 and 1 , where values at 0.5 signify signal levels equal to noise levels.

Anthropomorphic noise, such as power lines, power generators, moving vehicles and trains, can produce an incoherent noise mainly affecting frequencies above $1 \mathrm{~Hz}$. An ungrounded, barbed wire fence along Profile A did not influence the data. Noise sources were absent from Profiles B and E. Data along Profile C were noisier than the other profiles: A power line located over $10 \mathrm{~km}$ away from Profile $\mathrm{C}$ was the only known source of noise in the area, and we do not have explanations for the increased broadband noise on several soundings.

Several soundings were recorded at each station. The best sounding from each station is presented here and will be used in subsequent modeling of the data. The unedited data presented here are not rotated, but fixed at specific azimuths as acquired in the field (Table 1). The ExHy mode is the nominal TM and the EyHx the TE mode. Scalar data as calculated in the Imagem presentational software (Geometrics, 2000) are presented. For each station, three separate plots are given in Appendix A:

1. Apparent Resistivity for the TM and TE modes at a fixed direction.

2. Impedance Phase for the TM and TE modes at a fixed direction.

3. E-Predicted Coherencies are $($ ExHy * Conj(ExHy) $) /(\mathrm{Hy} * \operatorname{Conj}(\mathrm{Hy}) *$ Ex * Conj(Ex)) and (EyHx * Conj(EyHx) $) /\left(\mathrm{Hx}^{*} \operatorname{Conj}(\mathrm{Hx}) *\right.$ Ey * Conj(Ey)), where Conj indicates complex conjugation.

Electronic data are available upon request in two formats: Geometrics Z Impedance files (Geometrics, 2000; Appendix A) and the Electronic Data Interchange (EDI), which was established in 1987 by the Society of Exploration Geophysicists (SEG) as a standard format for the interchange of MT data (Wright, 1988). 


\section{Acknowledgments}

This study was performed in cooperation with the Southern Nevada Water Authority (SNWA) whose support is greatly appreciated. We are grateful to Janet Tilden for her help in the field, and to Gary Dixon, Jeff Johnston, and Ester Falgás Parra for contributing their expertise and advice. We also thank Ed Mankinen and Vicki Langenheim for their helpful reviews.

\section{References Cited}

Eberhart-Phillips, D., Stanley, W.D., Rodriguez, B.D. and Lutter, W.J., 1995, Surface seismic and electrical methods to detect fluids related to faulting: Journal of Geophysical Research, vol. 100, no. B7, p. 12,919-12,936.

Geometrics, 2000, Operation Manual for Stratagem systems running IMAGEM, Ver. 2.16: Geometrics, San Jose, California.

Harrill, J.R., and Prudic, D.E., 1998, Aquifer systems in the Great Basin region of Nevada, Utah and adjacent states - summary report: U.S. Geological Professional Paper, 1409A, 61p.

Keller, G.V., 1987, Rock and mineral properties, in Electromagnetic Methods in Applied Geophysics Theory: M.N. Nabighian, Ed., Society of Exploration Geophysicists, Tulsa, Oklahoma, v.1, p. 13-51.

Keller, G.V., 1989, Electrical properties, in Carmichael, R.S., Ed., Practical handbook of physical properties of rocks and minerals: CRC Press, Boca Raton, Florida, p. 359-427.

Mankinen, E.A., Roberts, C.W., McKee, E.H., Chuchel, B.A., and Moring, B.C., 2006, Geophysical data from the Spring and Snake valleys area, Nevada and Utah: U.S. Geological Survey Open-File Report 2006-1160, 42 p. 
Palacky, G.J., 1987, Resistivity characteristics of geologic targets, in Electromagnetic Methods in Applied Geophysics Theory: M.N. Nabighian, Ed., Society of Exploration Geophysicists, Tulsa, Oklahoma, vol. 1, p. 53-129.

Scheirer, D.S., 2005, Gravity studies of Cave, Dry Lake, and Delamar Valleys, east-central Nevada: U.S. Geological Survey Open File Report, 2005-1339, 36p.

Telford, W.M., Geldart, L.P.., and Sheriff, R.E., 1991, Applied Geophysics (2 ${ }^{\text {nd }}$ Edition): Cambridge University Press, 790 p.

Vozoff, K., 1991, The magnetotelluric method, in Electromagnetic methods in applied geophysics: M.N. Nabighian, Ed., Society of Exploration Geophysicists, Tulsa, Oklahoma, vol. 2, part B, p. $641-711$.

Wright, D.E., 1988, The SEG Standard for Magnetotelluric Data: presented at the Society of Exploration Geophysicists Annual Meeting, November. Available through http://www.geophysics.dias.ie/mtnet/docs/ediformat.txt.

Zonge, K.L. and Hughes, L.J., 1991, Controlled source audio-frequency magnetotellurics, in Electromagnetic methods in applied geophysics: M.N. Nabighian, Ed., Society of Exploration Geophysicists, Tulsa, Oklahoma, vol. 2, part B, p. 713-809. 


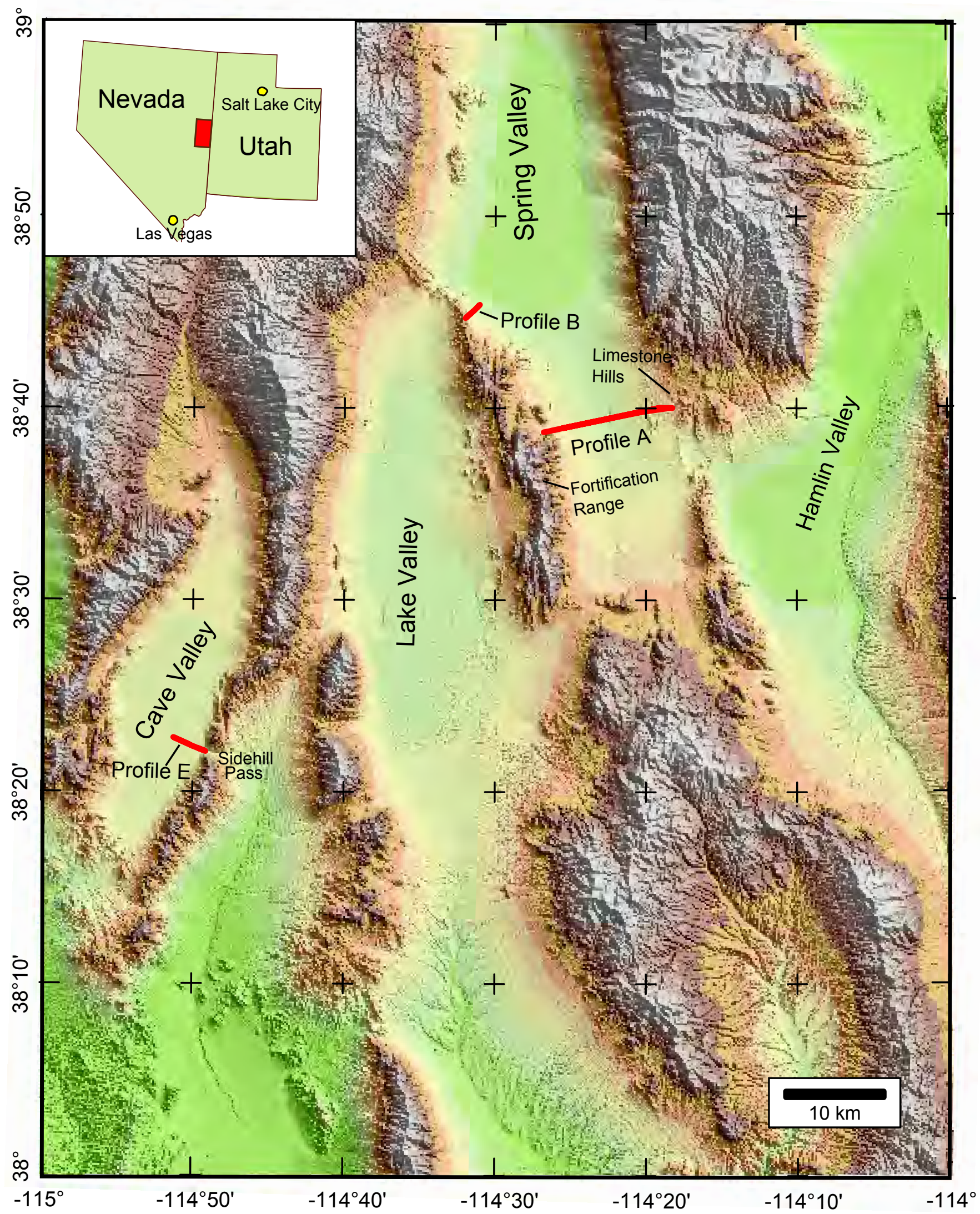

Figure $1 \mathrm{~A}$. Topographic map of study area showing location of profiles $A, B$, and $E$. 


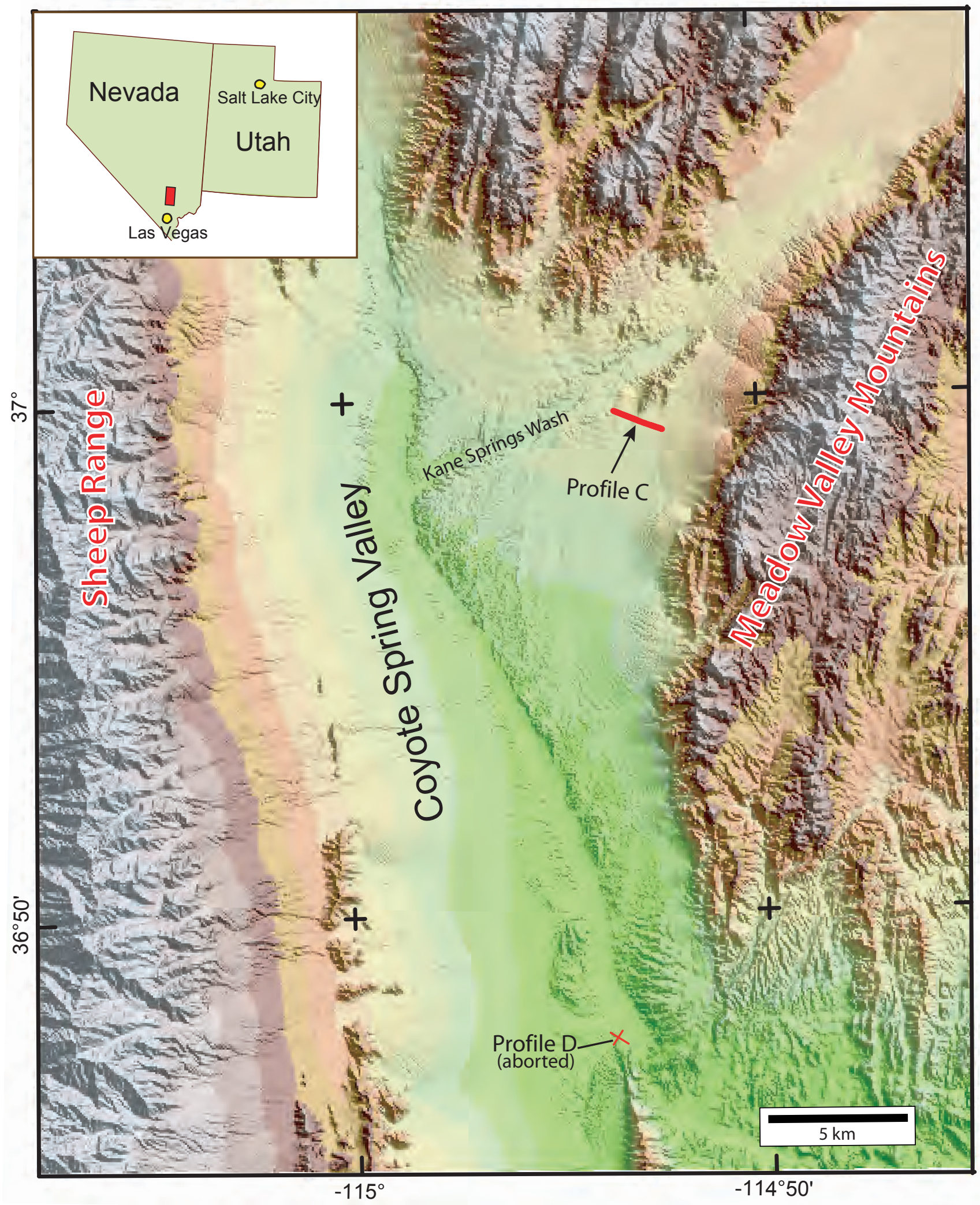

Figure 1B. Topographic map of study area showing location of profile C. 
Table 1. Description of AMT profiles.

[Azimuth refers to the magnetic orientation of the x-direction, which remained fixed throughout each profile]

\begin{tabular}{|c|c|c|c|c|l|}
\hline Profile & Length & $\begin{array}{c}\text { Azimuth } \\
\text { (x-direction) }\end{array}$ & $\begin{array}{c}\text { Station } \\
\text { Spacing }\end{array}$ & $\begin{array}{c}\text { Number of } \\
\text { Stations }\end{array}$ & \multicolumn{1}{|c|}{ Comments } \\
\hline A & $12.6 \mathrm{~km}$ & $80^{\circ}$ & $200 \mathrm{~m}$ & 64 & $\begin{array}{l}\text { Across southern Spring Valley from } \\
\text { the Fortification Range (west) to the } \\
\text { Limestone Hills (east). }\end{array}$ \\
\hline B & $2.2 \mathrm{~km}$ & $80^{\circ}$ & $200-400 \mathrm{~m}$ & 9 & $\begin{array}{l}\text { North of Fortification Range, west } \\
\text { side of Spring Valley }\end{array}$ \\
\hline C & $1.4 \mathrm{~km}$ & $100^{\circ}$ & $100-200 \mathrm{~m}$ & 9 & $\begin{array}{l}\text { Eastern Coyote Spring Valley near } \\
\text { Kane Springs Wash }\end{array}$ \\
\hline D & N/A & N/A & N/A & N/A & $\begin{array}{l}\text { Aborted due to power line } \\
\text { interference }\end{array}$ \\
\hline E & $3.0 \mathrm{~km}$ & $100^{\circ}$ & $200-400 \mathrm{~m}$ & 14 & Cave Valley west of Sidehill Pass \\
\hline
\end{tabular}

Table 2. Sounding numbers, locations and elevations of stations along four AMT

profiles.

[Distance indicates distance along profile in meters, where positive is east. Latitude and longitude are in NAD 27 decimal degrees. Universal Transverse Mercator (zone 11N) units are in meters. Horizontal locations are accurate to approximately $3 \mathrm{~m}$ (10 ft.) Station elevation is given in meters and feet and are accurate to approximately $7 \mathrm{~m}(23 \mathrm{ft}$.).]

\begin{tabular}{|c|c|c|c|c|c|c|c|}
\hline \multicolumn{7}{|c|}{ Profile A (SVNA) } \\
\hline Sounding & $\begin{array}{c}\text { Distance } \\
(\mathrm{m})\end{array}$ & Latitude & Longitude & $\begin{array}{c}\text { Easting } \\
(\mathrm{m})\end{array}$ & $\begin{array}{c}\text { Northing } \\
(\mathrm{m})\end{array}$ & $\begin{array}{c}\text { Elevation } \\
(\mathrm{m})\end{array}$ & $\begin{array}{c}\text { Elevation } \\
(\mathrm{ft})\end{array}$ \\
\hline 4 & 0 & 38.6459 & -114.4465 & 722154 & 4280566 & 1898 & 6228 \\
\hline 5 & 200 & 38.6463 & -114.4440 & 722368 & 4280617 & 1891 & 6204 \\
\hline 6 & 400 & 38.6465 & -114.4420 & 722542 & 4280652 & 1888 & 6193 \\
\hline 7 & 600 & 38.6469 & -114.4398 & 722737 & 4280694 & 1884 & 6180 \\
\hline 8 & 800 & 38.6474 & -114.4375 & 722931 & 4280755 & 1880 & 6169 \\
\hline 10 & 1000 & 38.6477 & -114.4353 & 723126 & 4280799 & 1868 & 6128 \\
\hline 12 & 1200 & 38.6481 & -114.4330 & 723320 & 4280846 & 1878 & 6161 \\
\hline 15 & 1400 & 38.6484 & -114.4307 & 723519 & 4280891 & 1858 & 6097 \\
\hline 17 & 1600 & 38.6488 & -114.4285 & 723713 & 4280933 & 1847 & 6060 \\
\hline 54 & 1800 & 38.6492 & -114.4263 & 723902 & 4280990 & 1853 & 6078 \\
\hline 18 & 2000 & 38.6496 & -114.4240 & 724099 & 4281035 & 1848 & 6062 \\
\hline 56 & 2200 & 38.6499 & -114.4217 & 724296 & 4281078 & 1848 & 6063 \\
\hline 19 & 2400 & 38.6503 & -114.4195 & 724487 & 4281126 & 1839 & 6035 \\
\hline
\end{tabular}




\begin{tabular}{|c|c|c|c|c|c|c|c|}
\hline 57 & 2600 & 38.6507 & -114.4173 & 724682 & 4281178 & 1843 & 6045 \\
\hline 20 & 2800 & 38.6511 & -114.4150 & 724878 & 4281225 & 1836 & 6024 \\
\hline 58 & 3000 & 38.6515 & -114.4128 & 725072 & 4281270 & 1836 & 6025 \\
\hline 21 & 3200 & 38.6519 & -114.4105 & 725269 & 4281319 & 1832 & 6009 \\
\hline 59 & 3400 & 38.6523 & -114.4083 & 725461 & 4281369 & 1831 & 6007 \\
\hline 23 & 3600 & 38.6526 & -114.4060 & 725661 & 4281412 & 1825 & 5988 \\
\hline 60 & 3800 & 38.6530 & -114.4037 & 725856 & 4281457 & 1826 & 5992 \\
\hline 24 & 4000 & 38.6533 & -114.4015 & 726047 & 4281502 & 1818 & 5964 \\
\hline 61 & 4200 & 38.6537 & -114.3992 & 726243 & 4281550 & 1820 & 5970 \\
\hline 25 & 4400 & 38.6541 & -114.3970 & 726434 & 4281600 & 1811 & 5943 \\
\hline 62 & 4600 & 38.6545 & -114.3948 & 726630 & 4281647 & 1812 & 5944 \\
\hline 26 & 4800 & 38.6548 & -114.3925 & 726823 & 4281692 & 1799 & 5902 \\
\hline 63 & 5000 & 38.6552 & -114.3903 & 727017 & 4281742 & 1810 & 5937 \\
\hline 28 & 5200 & 38.6556 & -114.3880 & 727216 & 4281791 & 1806 & 5924 \\
\hline 64 & 5400 & 38.6560 & -114.3858 & 727405 & 4281836 & 1812 & 5946 \\
\hline 30 & 5600 & 38.6562 & -114.3835 & 727606 & 4281864 & 1805 & 5922 \\
\hline 65 & 5800 & 38.6567 & -114.3813 & 727795 & 4281932 & 1802 & 5911 \\
\hline 32 & 6000 & 38.6571 & -114.3790 & 727990 & 4281976 & 1823 & 5980 \\
\hline 67 & 6200 & 38.6574 & -114.3768 & 728187 & 4282020 & 1803 & 5915 \\
\hline 34 & 6400 & 38.6579 & -114.3745 & 728380 & 4282074 & 1803 & 5915 \\
\hline 68 & 6600 & 38.6582 & -114.3722 & 728578 & 4282117 & 1816 & 5959 \\
\hline 35 & 6800 & 38.6586 & -114.3700 & 728770 & 4282168 & 1815 & 5955 \\
\hline 69 & 7000 & 38.6590 & -114.3678 & 728963 & 4282213 & 1812 & 5946 \\
\hline 95 & 7200 & 38.6593 & -114.3655 & 729159 & 4282260 & 1828 & 5997 \\
\hline 70 & 7400 & 38.6597 & -114.3633 & 729350 & 4282309 & 1819 & 5967 \\
\hline 39 & 7600 & 38.6601 & -114.3610 & 729550 & 4282358 & 1826 & 5992 \\
\hline 71 & 7800 & 38.6605 & -114.3588 & 729742 & 4282403 & 1830 & 6005 \\
\hline 40 & 8000 & 38.6608 & -114.3565 & 729935 & 4282450 & 1832 & 6010 \\
\hline 72 & 8200 & 38.6612 & -114.3543 & 730133 & 4282494 & 1832 & 6012 \\
\hline 41 & 8400 & 38.6616 & -114.3520 & 730327 & 4282545 & 1834 & 6016 \\
\hline 74 & 8600 & 38.6620 & -114.3498 & 730520 & 4282593 & 1837 & 6028 \\
\hline 43 & 8800 & 38.6623 & -114.3475 & 730717 & 4282638 & 1843 & 6045 \\
\hline 75 & 9000 & 38.6627 & -114.3453 & 730909 & 4282685 & 1848 & 6062 \\
\hline 45 & 9200 & 38.6631 & -114.3430 & 731105 & 4282731 & 1853 & 6078 \\
\hline 77 & 9400 & 38.6635 & -114.3408 & 731297 & 4282781 & 1855 & 6086 \\
\hline 46 & 9600 & 38.6638 & -114.3385 & 731493 & 4282820 & 1860 & 6104 \\
\hline 78 & 9800 & 38.6642 & -114.3363 & 731688 & 4282872 & 1866 & 6121 \\
\hline 47 & 10000 & 38.6646 & -114.3336 & 731923 & 4282930 & 1872 & 6142 \\
\hline 80 & 10200 & 38.6649 & -114.3318 & 732076 & 4282965 & 1875 & 6152 \\
\hline 49 & 10400 & 38.6653 & -114.3295 & 732273 & 4283012 & 1878 & 6162 \\
\hline 82 & 10600 & 38.6657 & -114.3273 & 732468 & 4283059 & 1884 & 6180 \\
\hline 51 & 10800 & 38.6660 & -114.3250 & 732663 & 4283107 & 1888 & 6195 \\
\hline 83 & 11000 & 38.6664 & -114.3228 & 732858 & 4283151 & 1895 & 6218 \\
\hline 52 & 11200 & 38.6668 & -114.3205 & 733055 & 4283201 & 1896 & 6219 \\
\hline 84 & 11400 & 38.6671 & -114.3182 & 733254 & 4283242 & 1904 & 6247 \\
\hline 98 & 11600 & 38.6671 & -114.3158 & 733458 & 4283245 & 1913 & 6277 \\
\hline 87 & 11800 & 38.6671 & -114.3135 & 733663 & 4283248 & 1915 & 6282 \\
\hline 94 & 12000 & 38.6686 & -114.3121 & 733857 & 4283221 & 1959 & 6246 \\
\hline
\end{tabular}




\begin{tabular}{|c|c|c|c|c|c|c|c|}
\hline 88 & 12200 & 38.6671 & -114.3090 & 734054 & 4283260 & 1914 & 6279 \\
\hline 93 & 12400 & 38.6670 & -114.3067 & 734255 & 4283259 & 1929 & 6329 \\
\hline 102 & 12600 & 38.6670 & -114.3040 & 734489 & 4283264 & 1911 & 6270 \\
\hline \multicolumn{8}{|c|}{ Profile B (SVNB) } \\
\hline Sounding & $\begin{array}{l}\text { Distance } \\
(\mathrm{m})\end{array}$ & Latitude & Longitude & $\begin{array}{c}\text { Easting } \\
(\mathrm{m})\end{array}$ & $\begin{array}{l}\text { Northing } \\
\text { (m) }\end{array}$ & $\begin{array}{l}\text { Elevation } \\
(\mathrm{m})\end{array}$ & $\begin{array}{c}\text { Elevation } \\
(\mathrm{ft})\end{array}$ \\
\hline 107 & -200 & 38.7644 & -114.5394 & 713791 & 4293294 & 1805 & 5922 \\
\hline 103 & 0 & 38.7654 & -114.5375 & 713952 & 4293411 & 1789 & 5871 \\
\hline 108 & 200 & 38.7664 & -114.5356 & 714114 & 4293527 & 1790 & 5874 \\
\hline 104 & 400 & 38.7672 & -114.5335 & 714293 & 4293623 & 1783 & 5850 \\
\hline 109 & 600 & 38.7677 & -114.5314 & 714481 & 4293681 & 1770 & 5807 \\
\hline 105 & 800 & 38.7677 & -114.5280 & 714698 & 4293696 & 1769 & 5803 \\
\hline 106 & 1200 & 38.7676 & -114.5240 & 715118 & 4293691 & 1765 & 5792 \\
\hline 111 & 1600 & 38.7674 & -114.5195 & 715514 & 4293675 & 1764 & 5786 \\
\hline 112 & 2000 & 38.7672 & -114.5149 & 715911 & 4293665 & 1761 & 5777 \\
\hline \multicolumn{8}{|c|}{ Profile C (SVNC) } \\
\hline Sounding & $\begin{array}{c}\text { Distance } \\
(\mathrm{m})\end{array}$ & Latitude & Longitude & $\begin{array}{c}\text { Easting } \\
(\mathrm{m})\end{array}$ & $\begin{array}{l}\text { Northing } \\
(\mathrm{m})\end{array}$ & $\begin{array}{c}\text { Elevation } \\
(\mathrm{m})\end{array}$ & $\begin{array}{c}\text { Elevation } \\
(\mathrm{ft})\end{array}$ \\
\hline 16 & -600 & 36.9962 & -114.8911 & 687590 & 4096521 & 871 & 2856 \\
\hline 27 & -400 & 36.9955 & -114.8890 & 687778 & 4096450 & 876 & 2873 \\
\hline 19 & -200 & 36.9947 & -114.8869 & 687965 & 4096365 & 875 & 2870 \\
\hline 21 & 0 & 36.9944 & -114.8847 & 688165 & 4096333 & 863 & 2831 \\
\hline 33 & 100 & 36.9937 & -114.8842 & 688212 & 4096264 & 871 & 2857 \\
\hline 32 & 200 & 36.9933 & -114.8830 & 688314 & 4096217 & 887 & 2911 \\
\hline 23 & 400 & 36.9920 & -114.8815 & 688456 & 4096075 & 888 & 2915 \\
\hline 25 & 600 & 36.9918 & -114.8791 & 688668 & 4096058 & 875 & 2870 \\
\hline 30 & 800 & 36.9912 & -114.8770 & 688854 & 4095993 & 882 & 2895 \\
\hline \multicolumn{8}{|c|}{ Profile E (SVNE) } \\
\hline Sounding & $\begin{array}{l}\text { Distance } \\
(\mathrm{m})\end{array}$ & Latitude & Longitude & $\begin{array}{c}\text { Easting } \\
(\mathrm{m})\end{array}$ & $\begin{array}{l}\text { Northing } \\
(\mathrm{m})\end{array}$ & $\begin{array}{l}\text { Elevation } \\
(\mathrm{m})\end{array}$ & $\begin{array}{c}\text { Elevation } \\
(\mathrm{ft})\end{array}$ \\
\hline 16 & -1800 & 38.3802 & -114.8546 & 687319 & 4250168 & 1819 & 5972 \\
\hline 14 & -1400 & 38.3787 & -114.8503 & 687698 & 4250011 & 1821 & 5979 \\
\hline 13 & -1000 & 38.3772 & -114.8462 & 688063 & 4249858 & 1820 & 5976 \\
\hline 12 & -800 & 38.3765 & -114.8441 & 688243 & 4249782 & 1823 & 5986 \\
\hline 10 & -600 & 38.3758 & -114.8421 & 688426 & 4249711 & 1835 & 6024 \\
\hline 7 & -400 & 38.3750 & -114.8400 & 688612 & 4249628 & 1820 & 5976 \\
\hline 41 & -200 & 38.3743 & -114.8378 & 688799 & 4249554 & 1825 & 5991 \\
\hline 4 & 0 & 38.3734 & -114.8358 & 688978 & 4249459 & 1820 & 5974 \\
\hline 39 & 200 & 38.3727 & -114.8337 & 689163 & 4249385 & 1826 & 5996 \\
\hline 19 & 400 & 38.3721 & -114.8316 & 689352 & 4249319 & 1825 & 5990 \\
\hline 23 & 600 & 38.3714 & -114.8295 & 689539 & 4249249 & 1821 & 5978 \\
\hline 36 & 800 & 38.3708 & -114.8273 & 689729 & 4249180 & 1822 & 5982 \\
\hline 26 & 1000 & 38.3701 & -114.8253 & 689910 & 4249110 & 1828 & 6002 \\
\hline 27 & 1200 & 38.3694 & -114.8231 & 690100 & 4249042 & 1834 & 6022 \\
\hline
\end{tabular}




\section{Appendix}

\section{A. Sounding curves}

The "Audiomagnetotelluric Data" section in this report contains an explanation for three separate plots for each station:

1. Apparent Resistivity for $\mathrm{x}$-directed profile line such that the nominal TM mode is ExHy (red diamond) and TE is EyHx (blue square).

2. Impedance Phase for $\mathrm{x}$-directed profile line such that the nominal TM mode is ExHy (red diamond) and TE is EyHx (blue square).

3. Multiple E-Predicted Coherencies, defined as $($ ExHy * Conj(ExHy) $) /(\mathrm{Hy} * \mathrm{Conj}(\mathrm{Hy}) *$ Ex * Conj(Ex)) (red diamond) and $(\mathrm{EyHx} * \mathrm{Conj}(\mathrm{EyHx})) /(\mathrm{Hx} * \mathrm{Conj}(\mathrm{Hx}) * \mathrm{Ey} *$ Conj(Ey)) (blue square)

Sounding curves are named by profile name, distance along the profile $(\mathrm{m})$ and sounding number, respectively. Note that for the distance along the profile, the positive direction trends eastward and the negative direction trends westward. 
SVNA 00000m 004 - Scalar Res., Coherency, Phase (diamond=ExHy; square=EyHx)

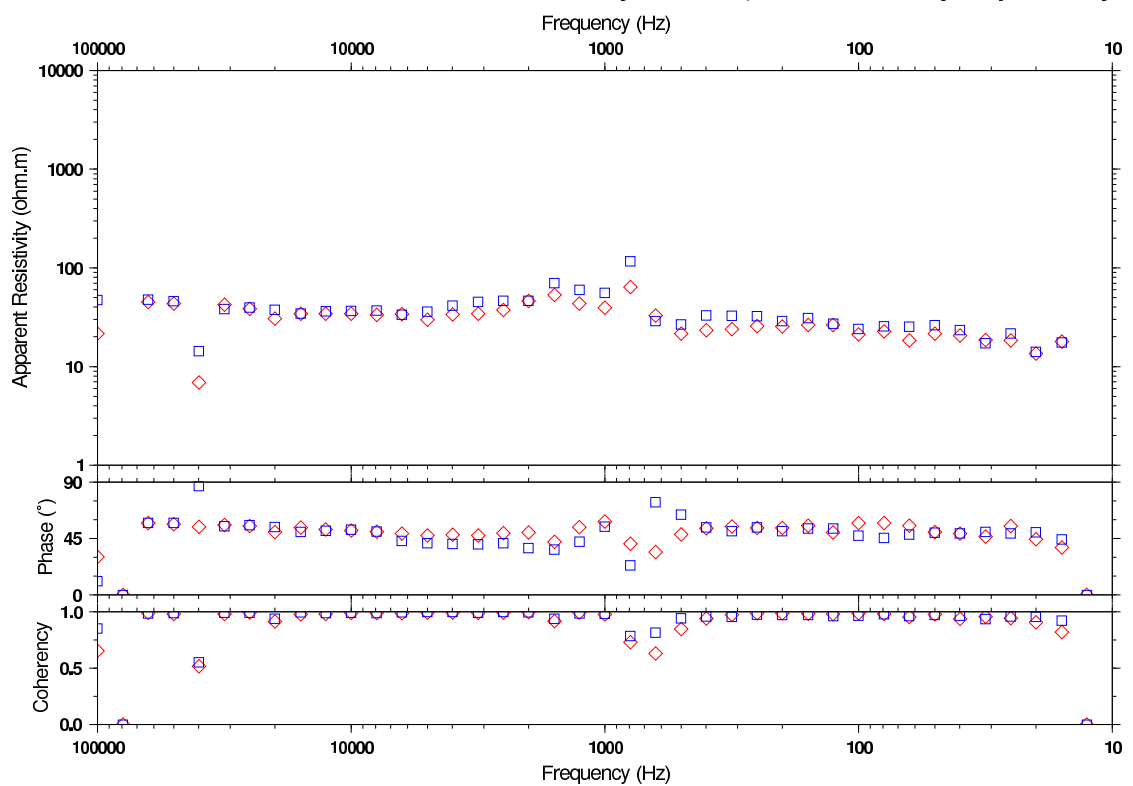

SVNA 00200m 005 - Scalar Res., Coherency, Phase (diamond=ExHy; square=EyHx)

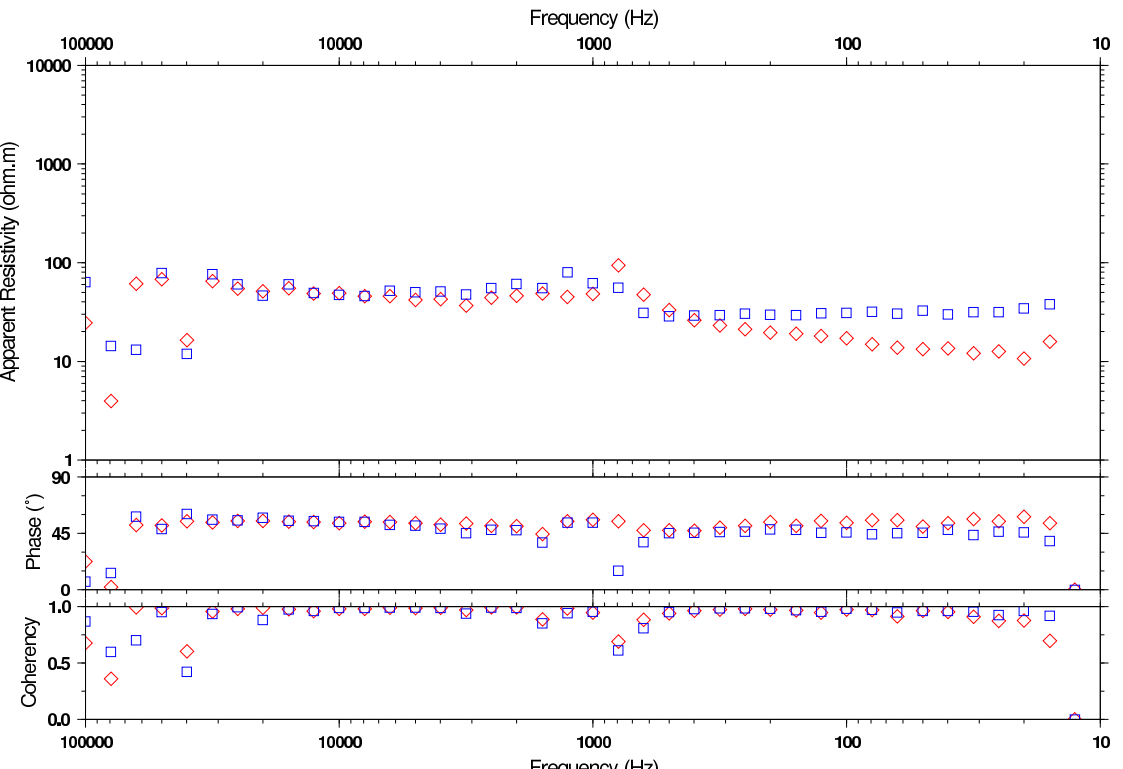

SVNA 00400m 006 - Scalar Res., Coherency, Phase (diamond=ExHy; square=EyHx)

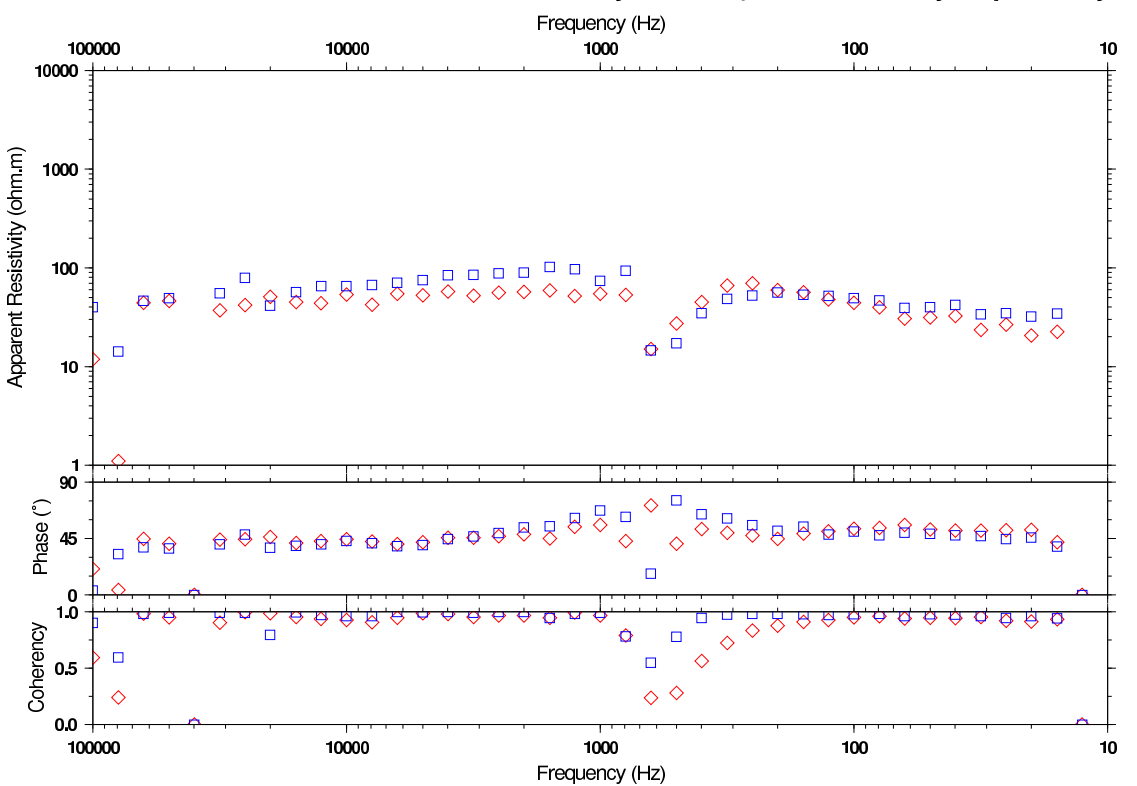

SVNA 00600m 007 - Scalar Res., Coherency, Phase (diamond=ExHy; square=EyHx)

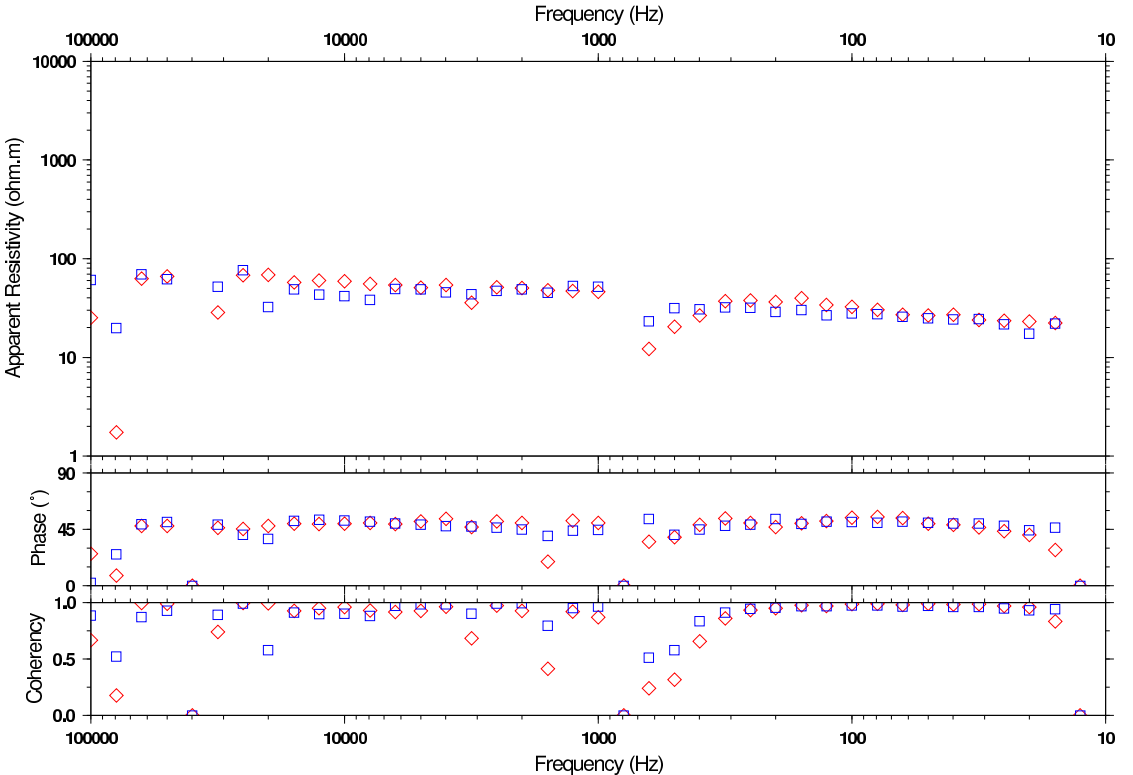


SVNA 00800m 008 - Scalar Res., Coherency, Phase (diamond=ExHy; square=EyHx)

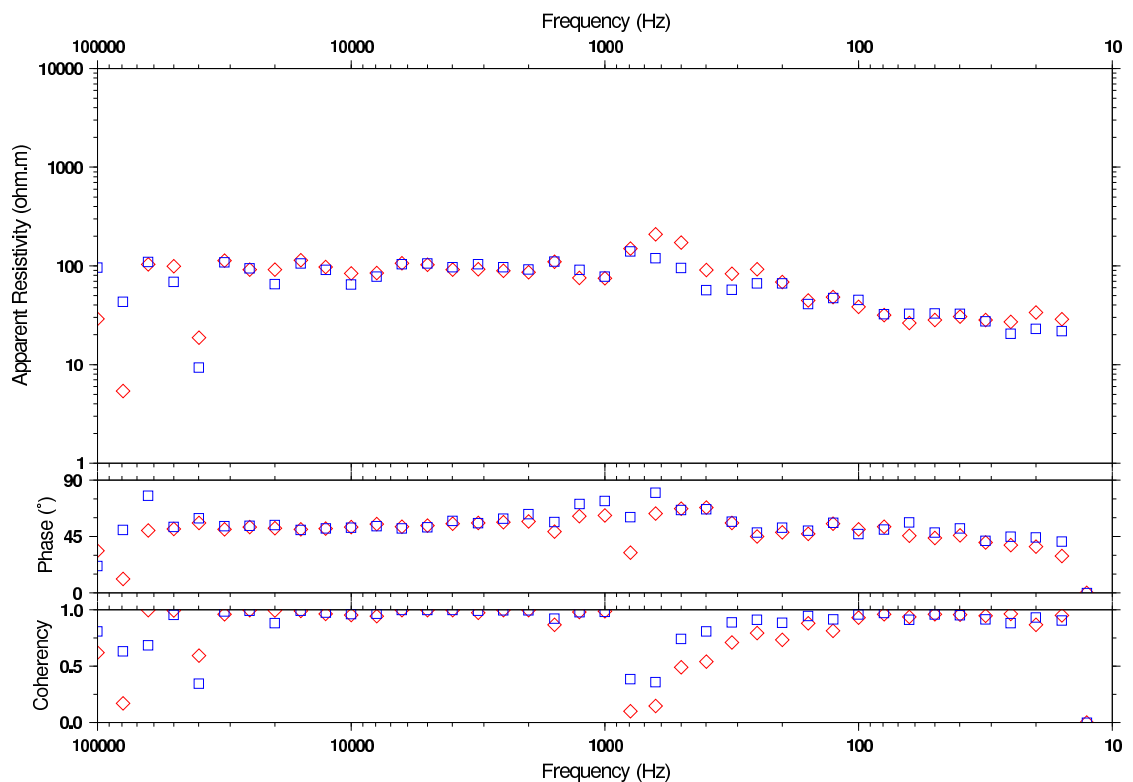

SVNA 01000m 010 - Scalar Res., Coherency, Phase (diamond=ExHy; square=EyHx)

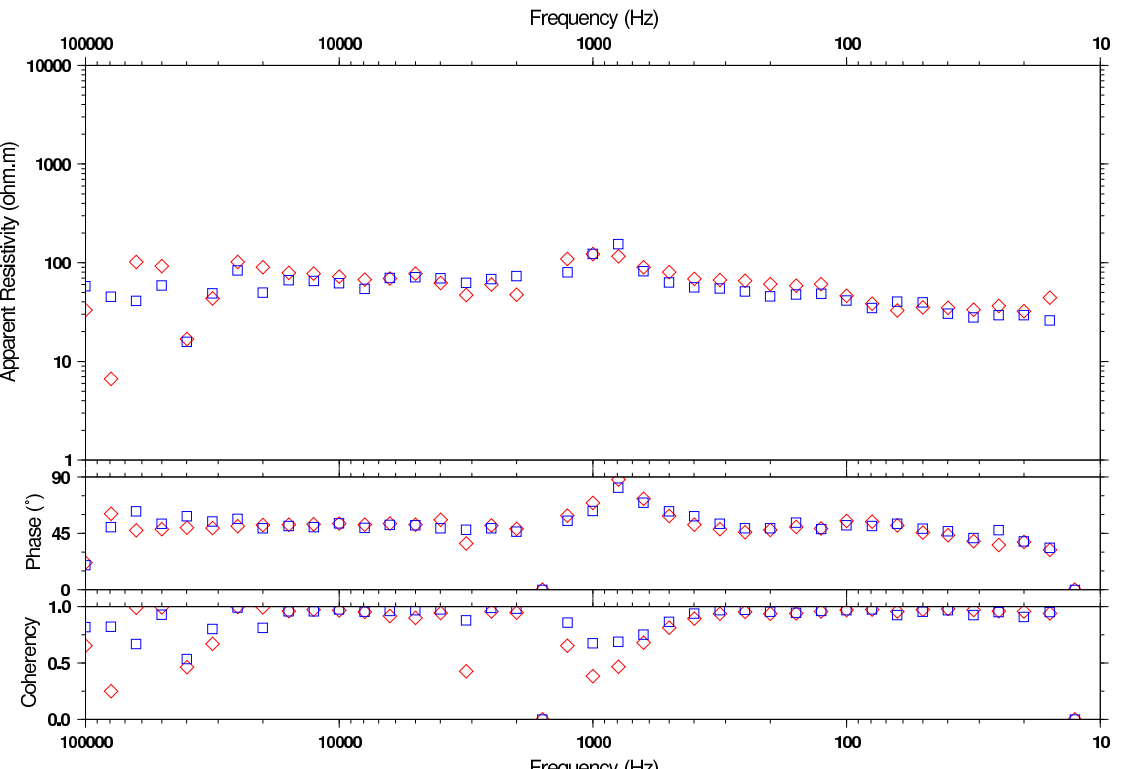

SVNA 01200m 012 - Scalar Res., Coherency, Phase (diamond=ExHy; square=EyHx)

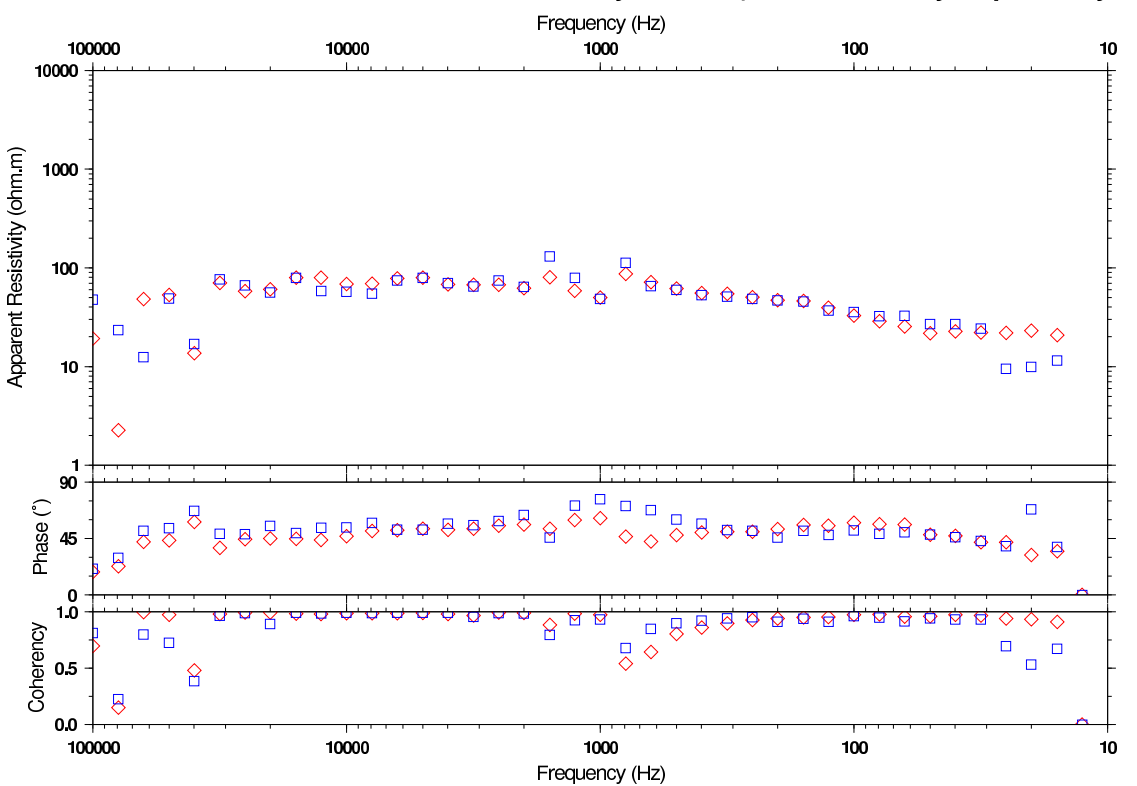

SVNA 01400m 015 - Scalar Res., Coherency, Phase (diamond=ExHy; square=EyHx)

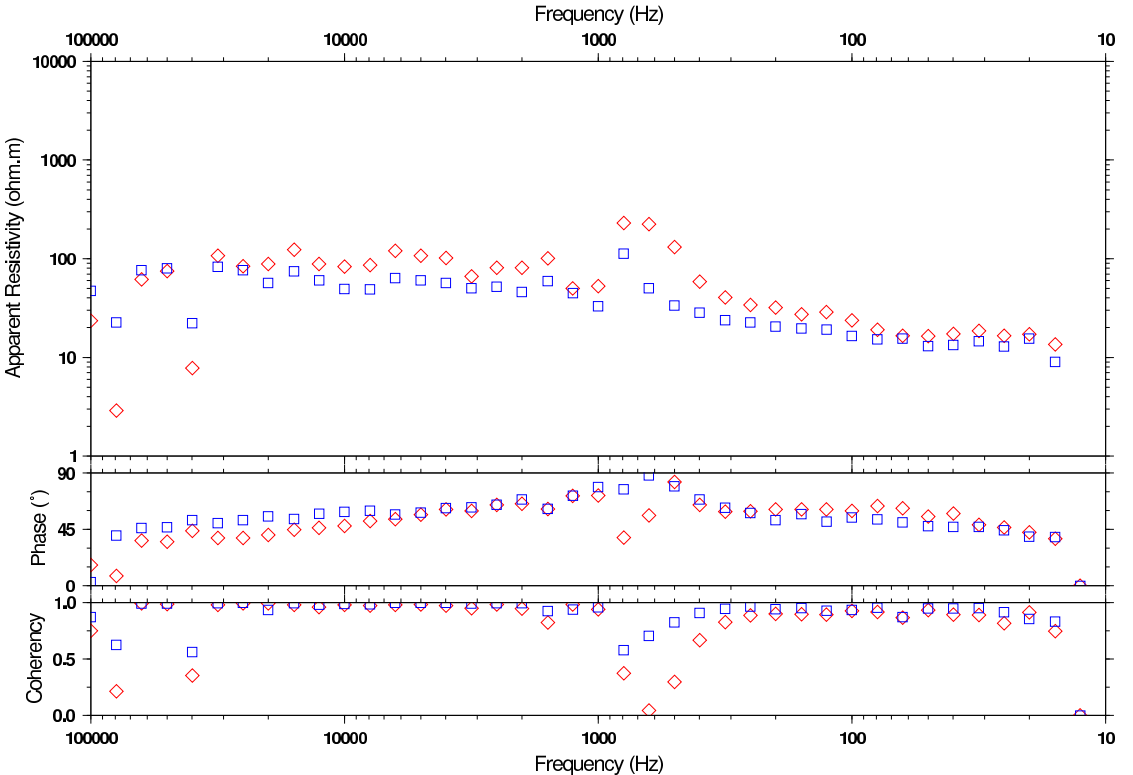


SVNA 01600m 017 - Scalar Res., Coherency, Phase (diamond=ExHy; square=EyHx)

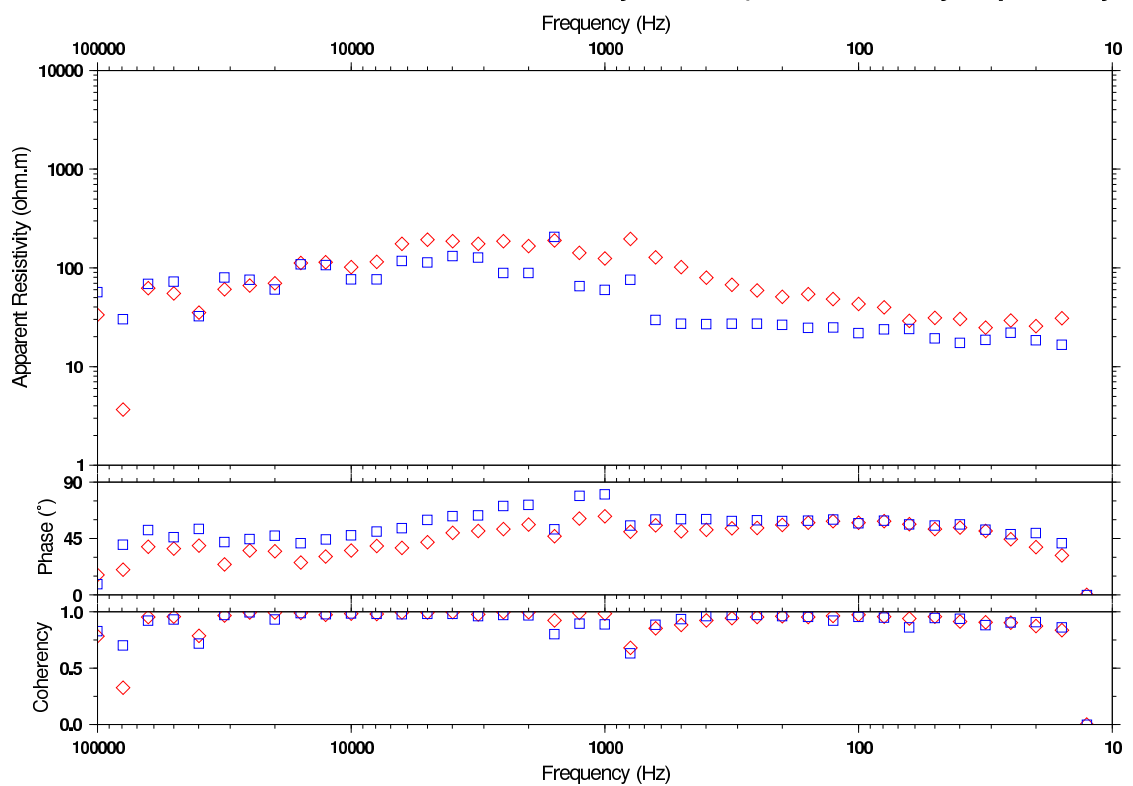

SVNA 01800m 054 - Scalar Res., Coherency, Phase (diamond=ExHy; square=EyHx)

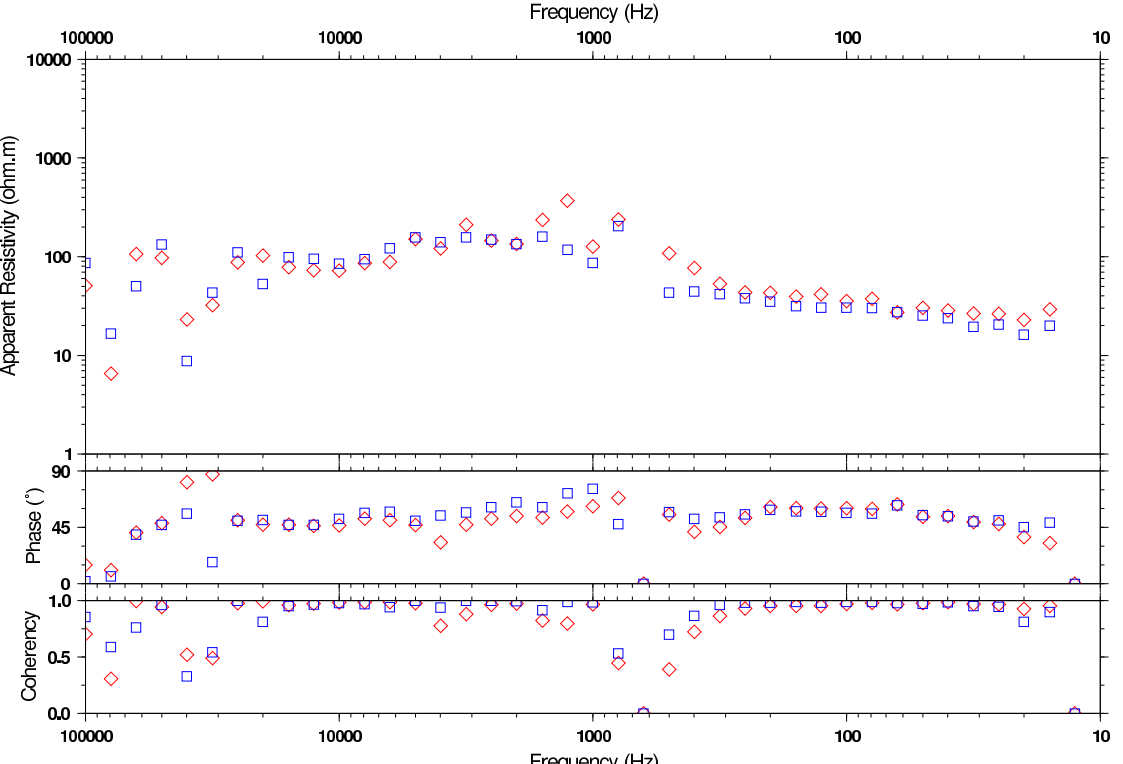

SVNA 02000m 018 - Scalar Res., Coherency, Phase (diamond=ExHy; square=EyHx)

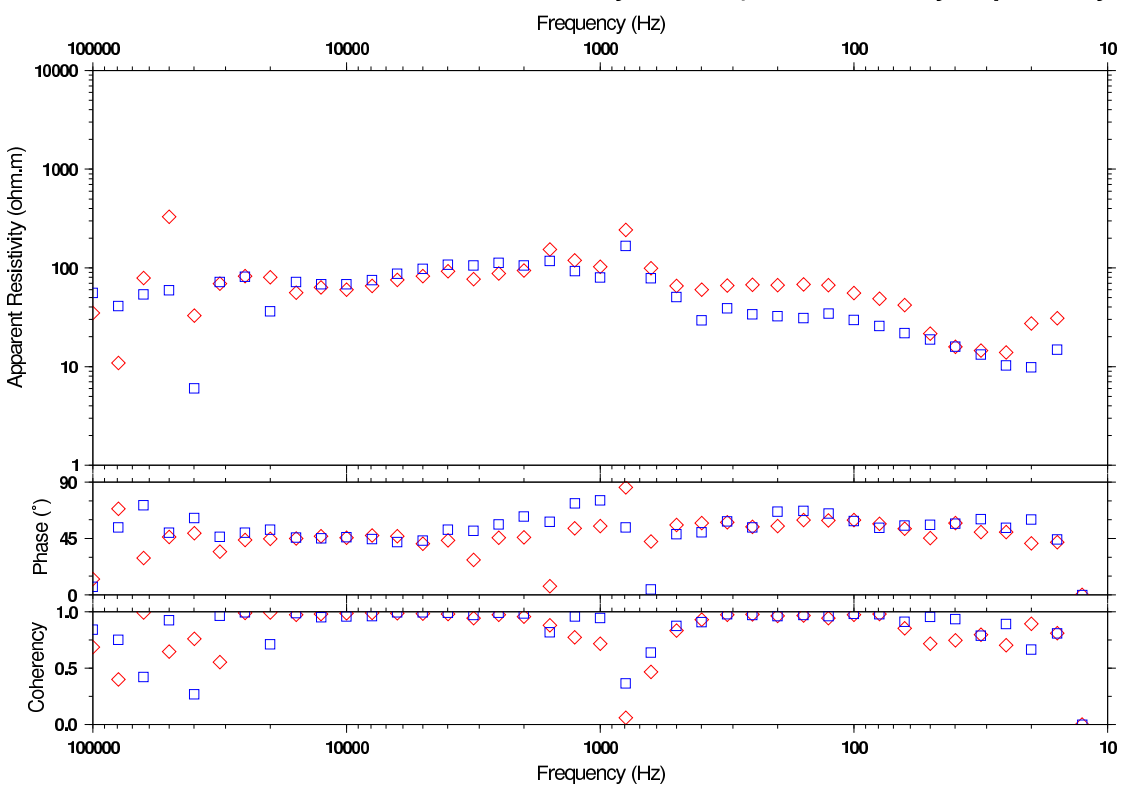

SVNA 02200m 056 - Scalar Res., Coherency, Phase (diamond=ExHy; square=EyHx)

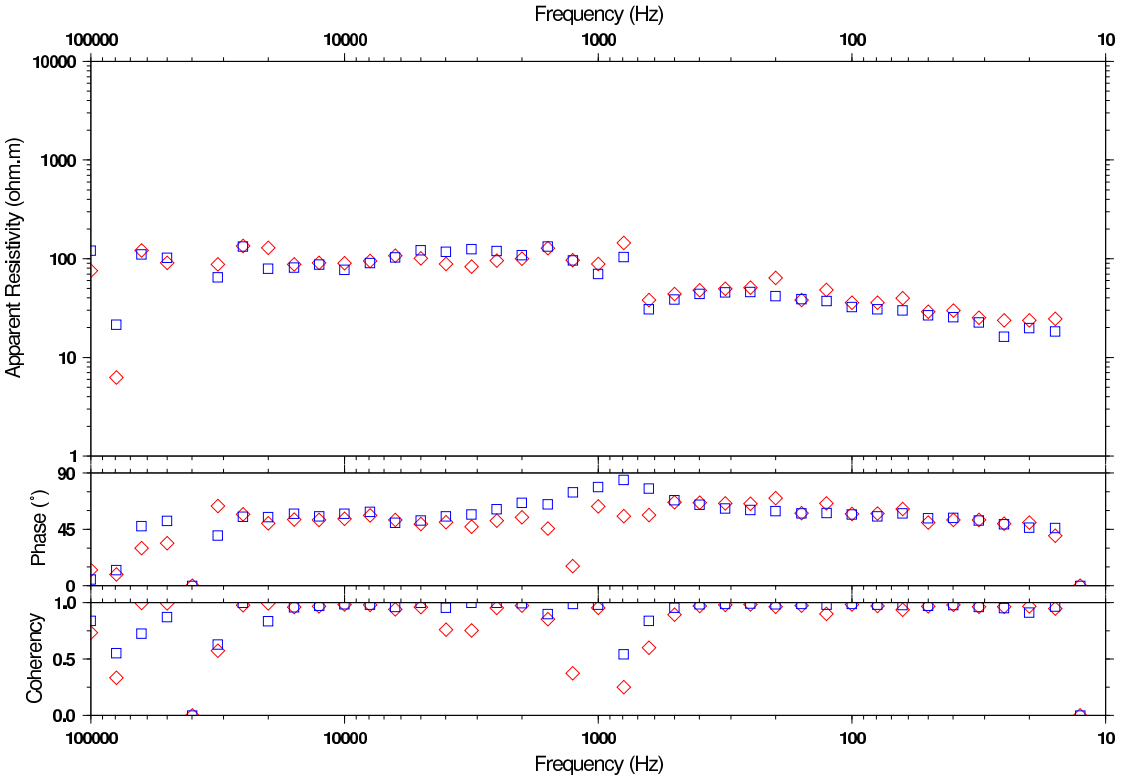


SVNA 02400m 019 - Scalar Res., Coherency, Phase (diamond=ExHy; square=EyHx)

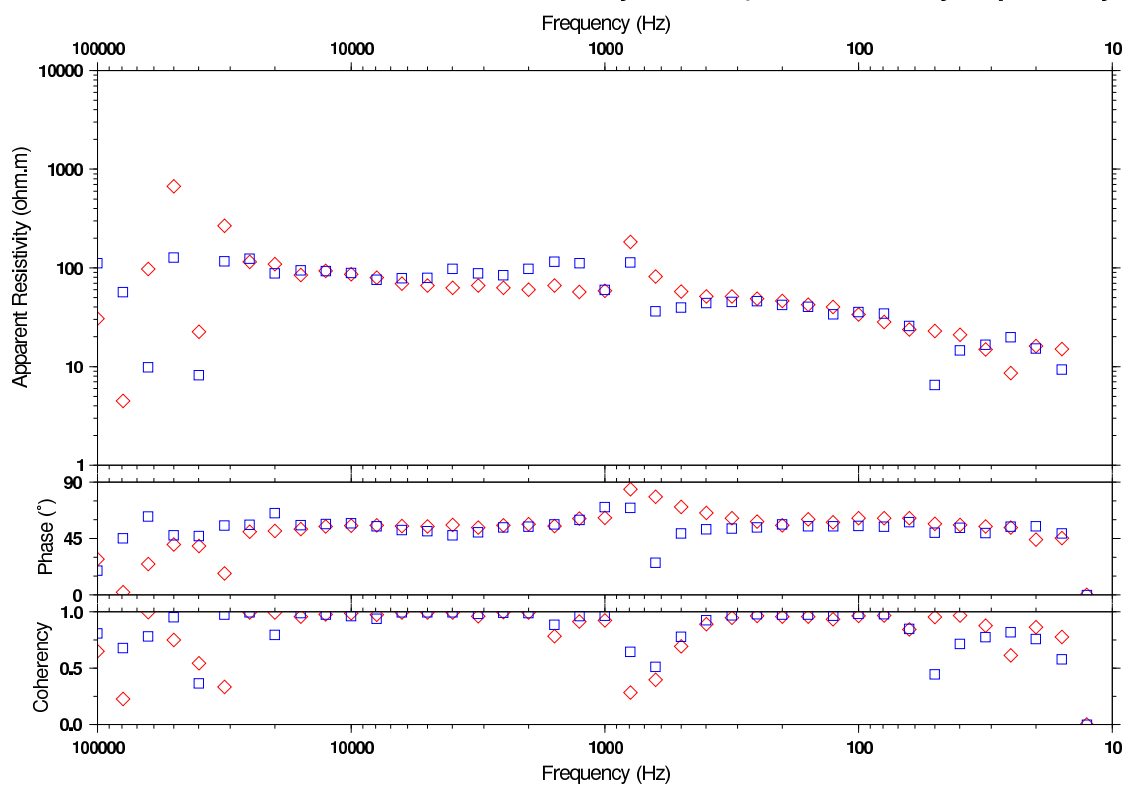

SVNA 02600m 057 - Scalar Res., Coherency, Phase (diamond=ExHy; square=EyHx)

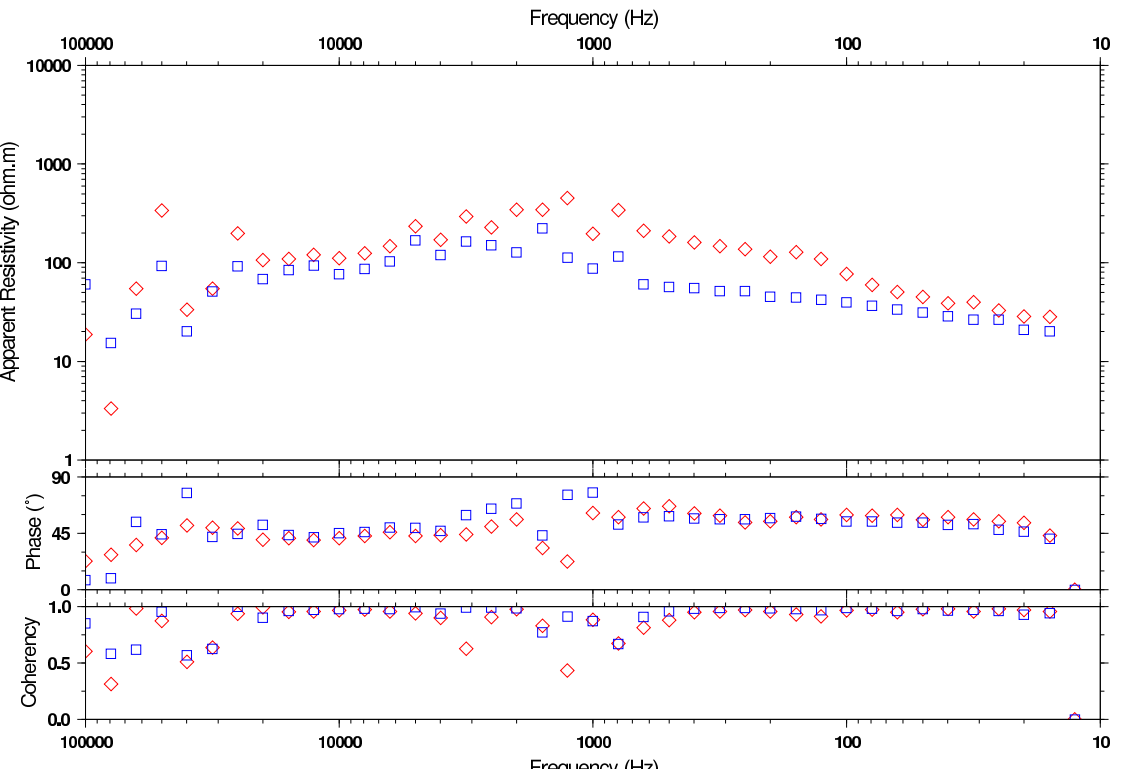

SVNA 02800m 020 - Scalar Res., Coherency, Phase (diamond=ExHy; square=EyHx)

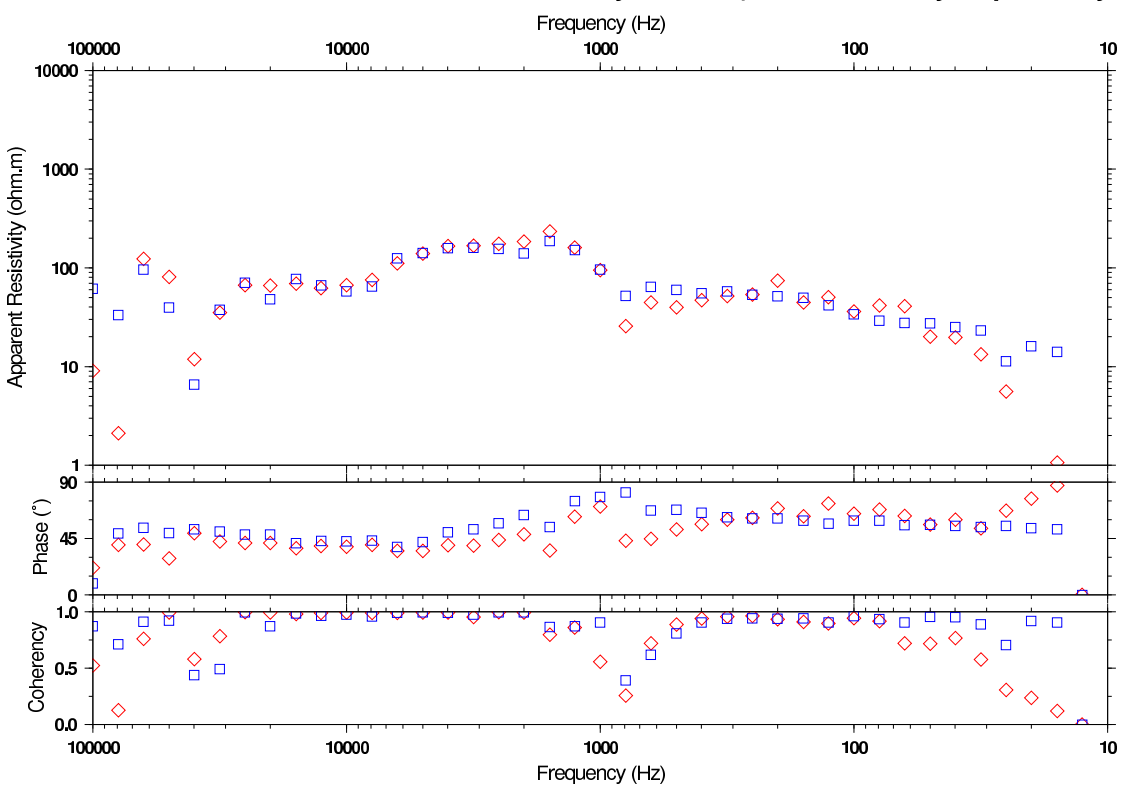

SVNA 03000m 058 - Scalar Res., Coherency, Phase (diamond=ExHy; square=EyHx)

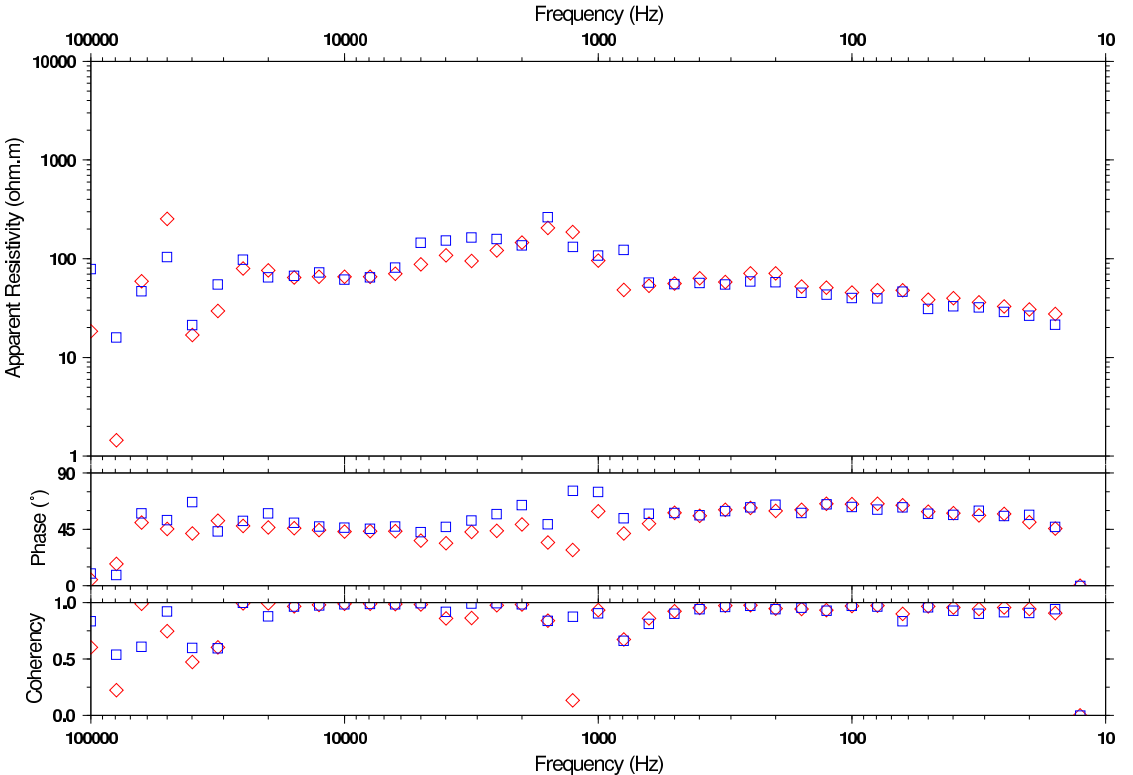


SVNA 03200m 021 - Scalar Res., Coherency, Phase (diamond=ExHy; square=EyHx)

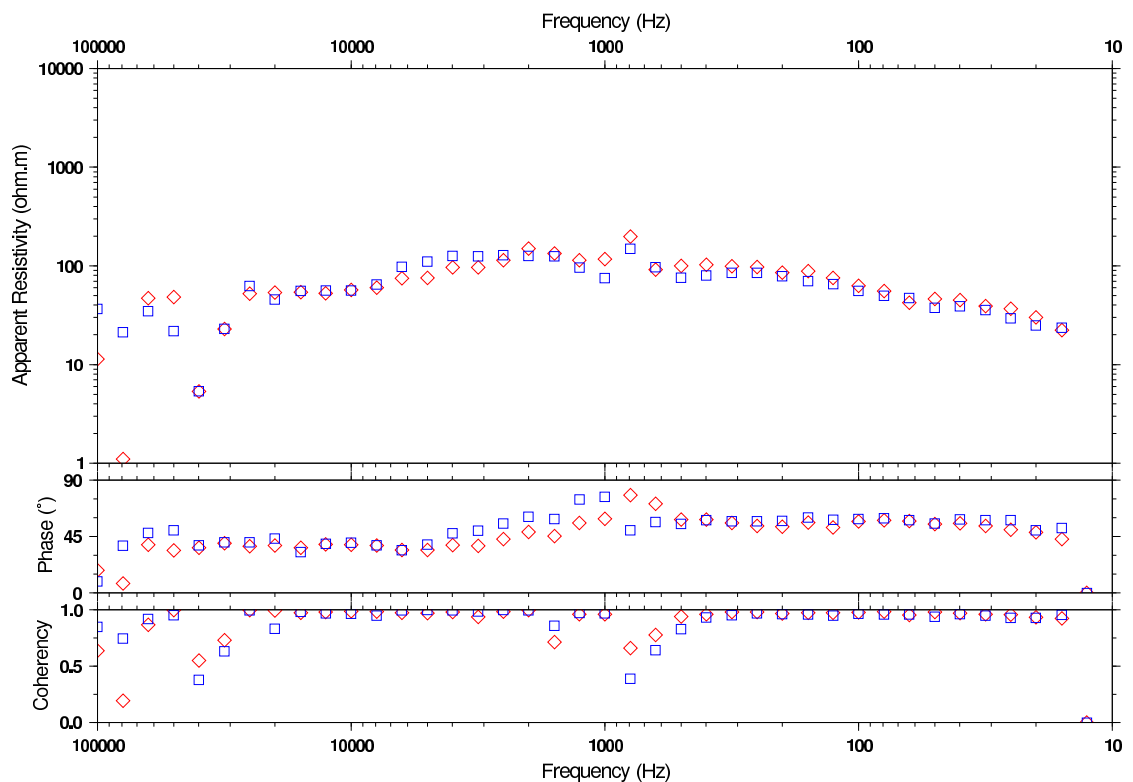

SVNA 03400m 059 - Scalar Res., Coherency, Phase (diamond=ExHy; square=EyHx)

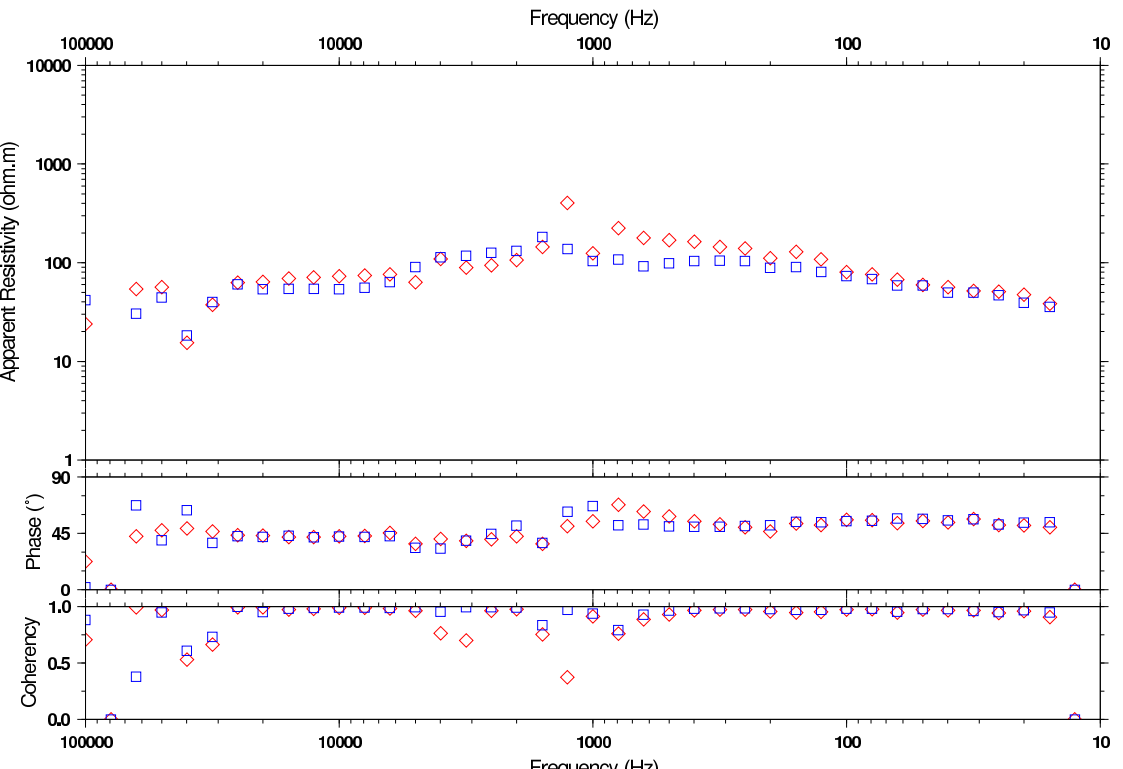

SVNA 03600m 023 - Scalar Res., Coherency, Phase (diamond=ExHy; square=EyHx)

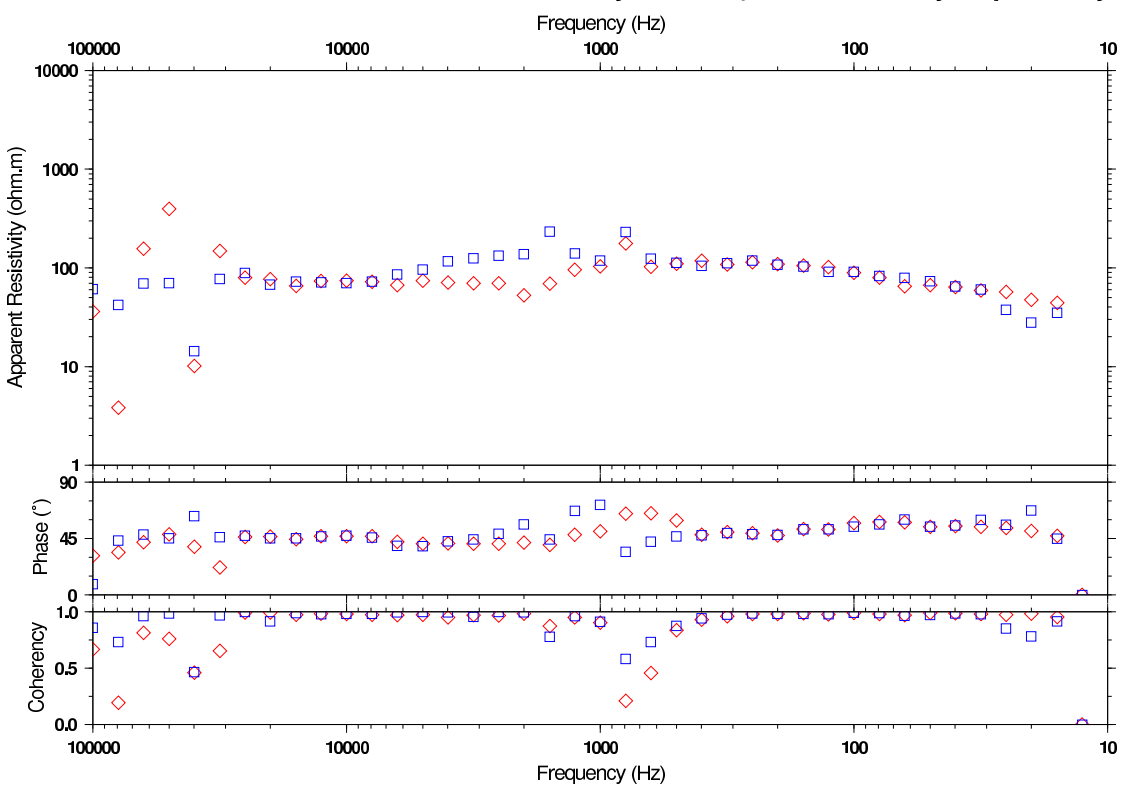

SVNA 03800m 060 - Scalar Res., Coherency, Phase (diamond=ExHy; square=EyHx)

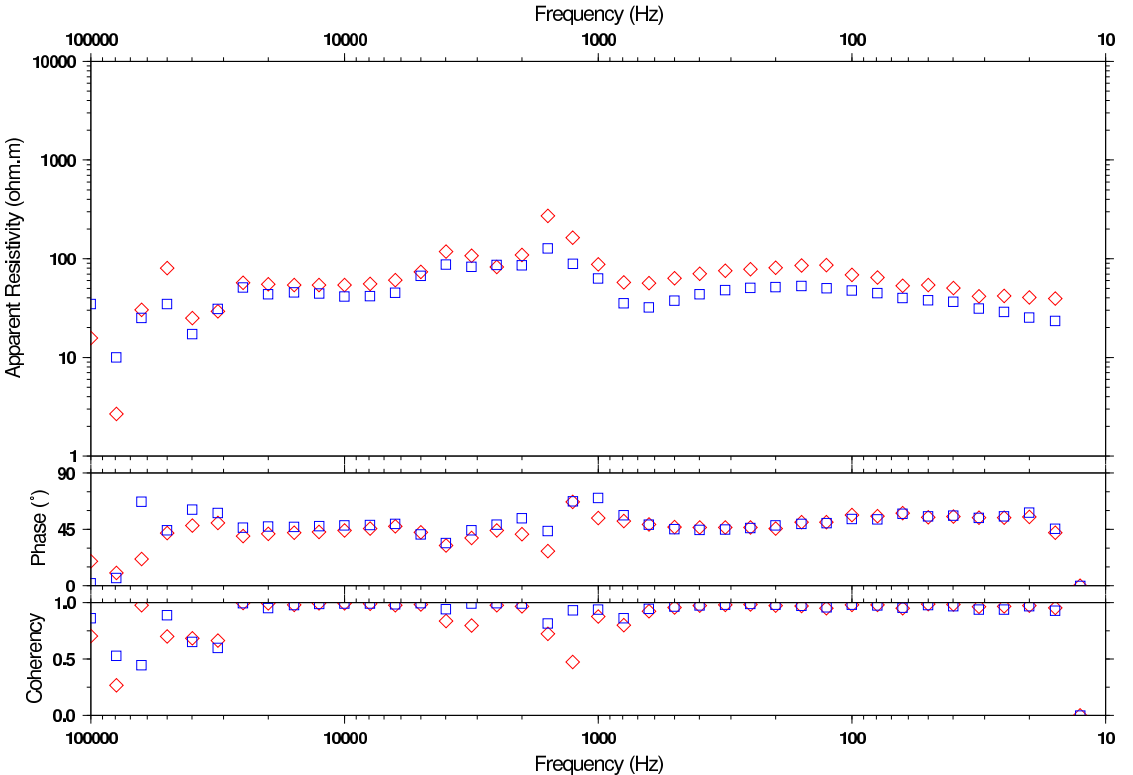


SVNA 04000m 024 - Scalar Res., Coherency, Phase (diamond=ExHy; square=EyHx)

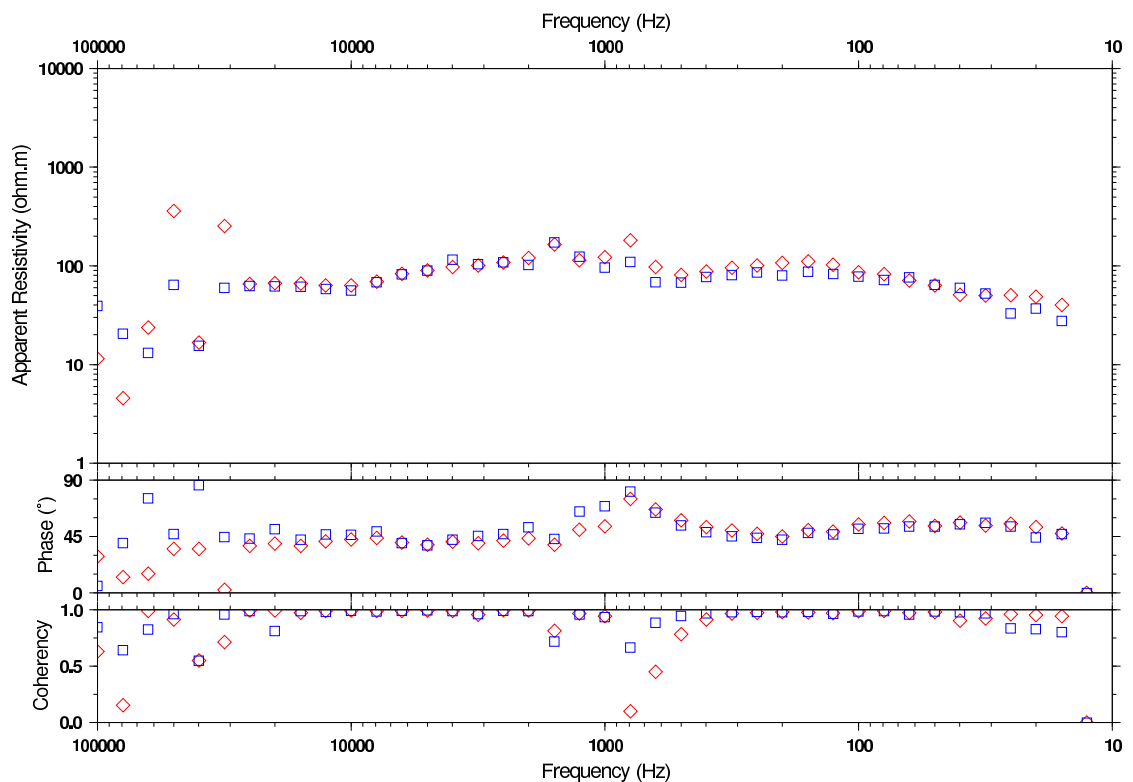

SVNA 04200m 061 - Scalar Res., Coherency, Phase (diamond=ExHy; square=EyHx)

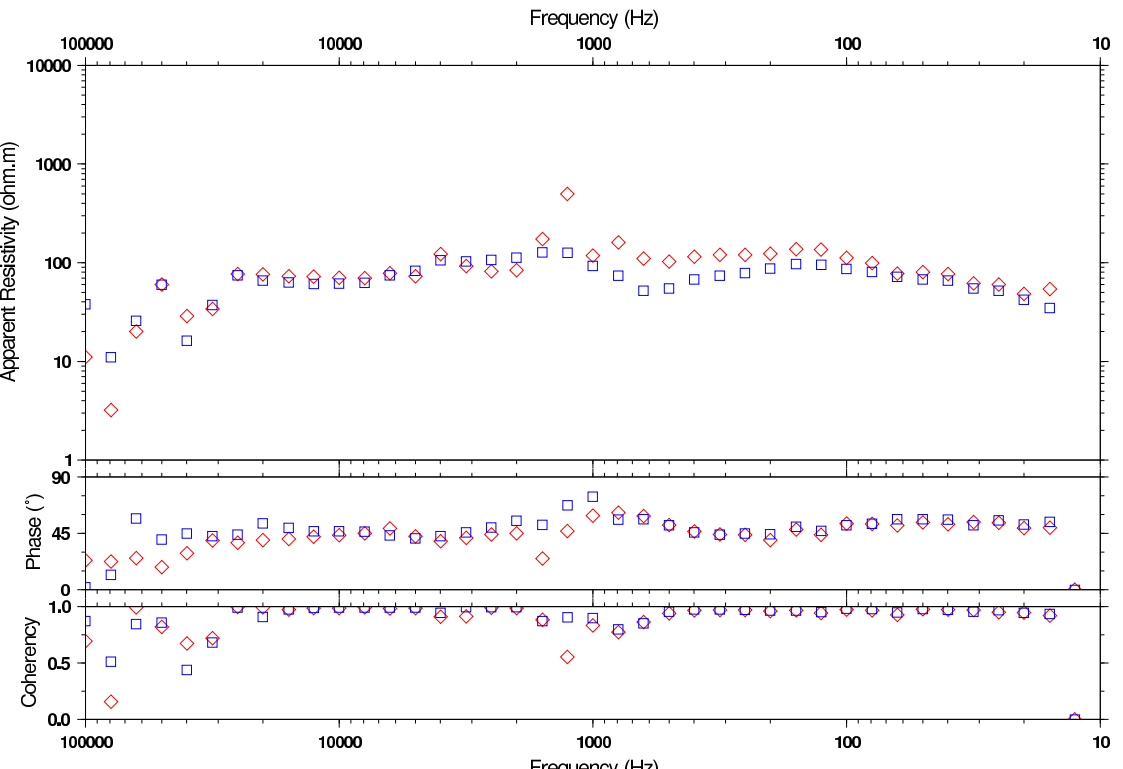

SVNA 04400m 025 - Scalar Res., Coherency, Phase (diamond=ExHy; square=EyHx)

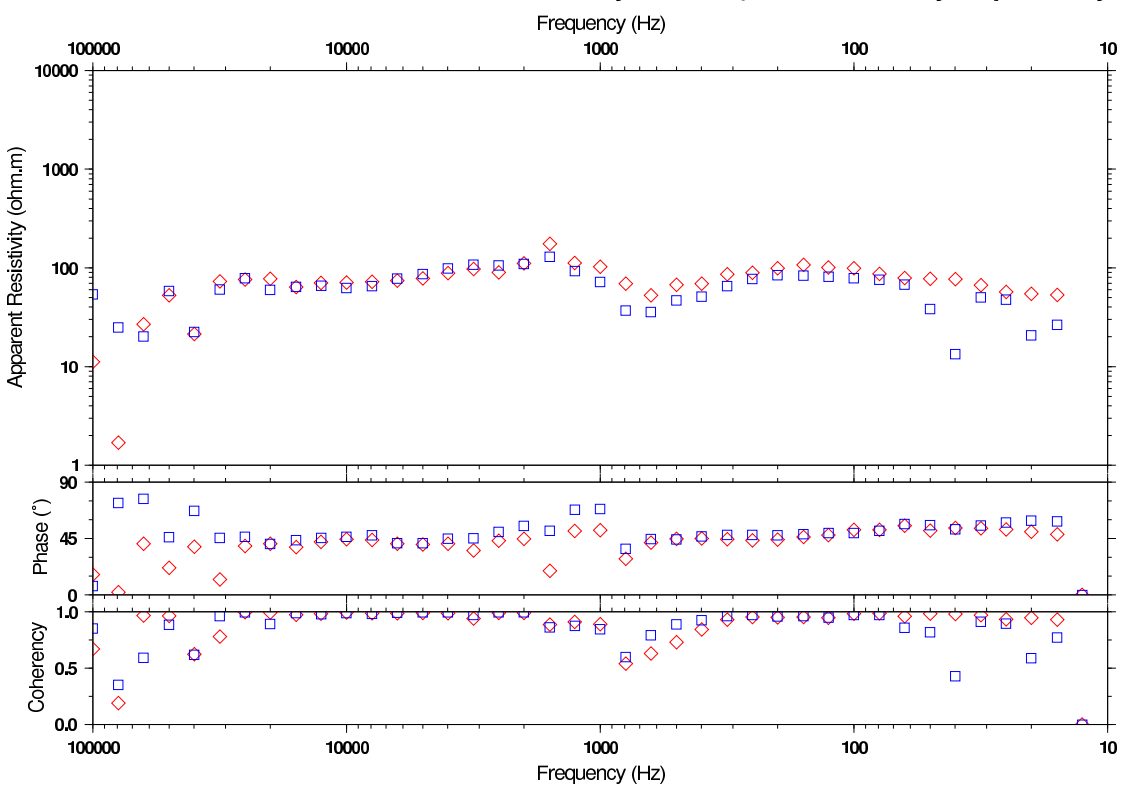

SVNA 04600m 062 - Scalar Res., Coherency, Phase (diamond=ExHy; square=EyHx)

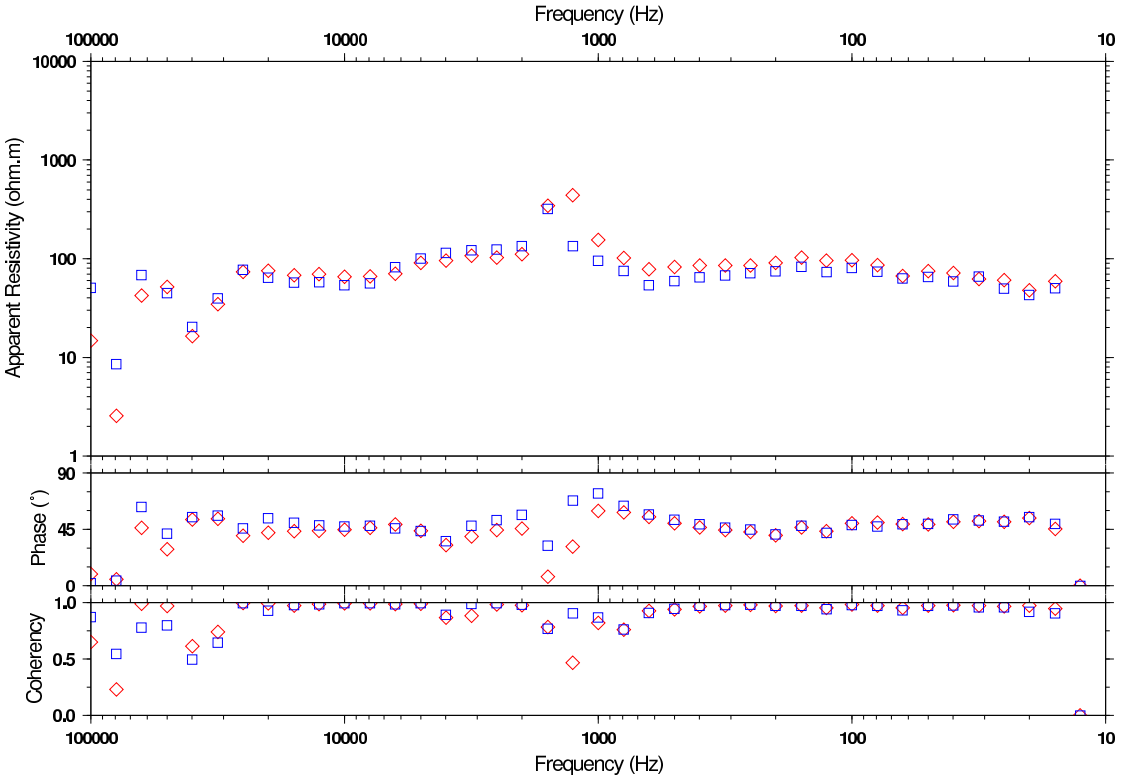




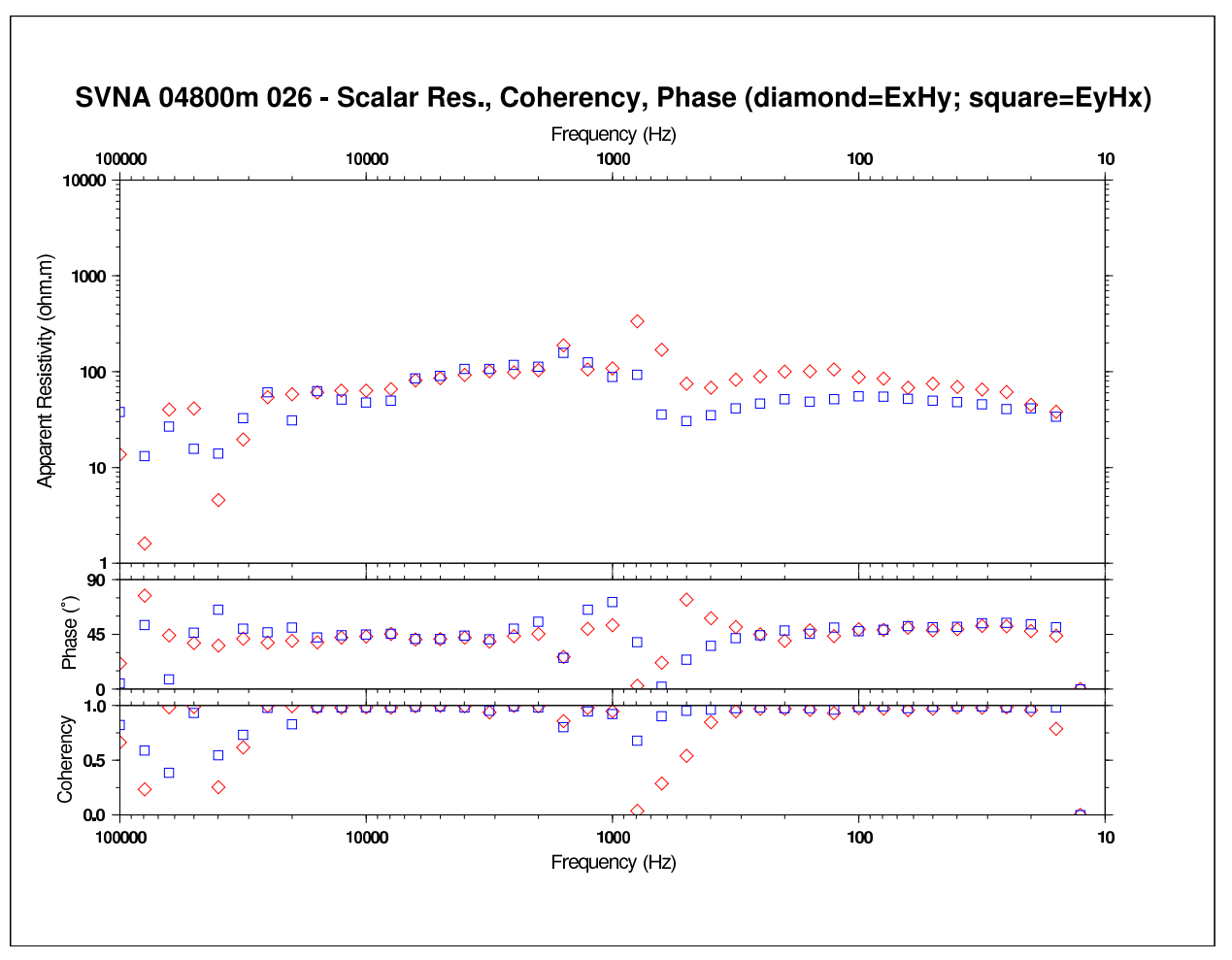

SVNA 05200m 028 - Scalar Res., Coherency, Phase (diamond=ExHy; square=EyHx)

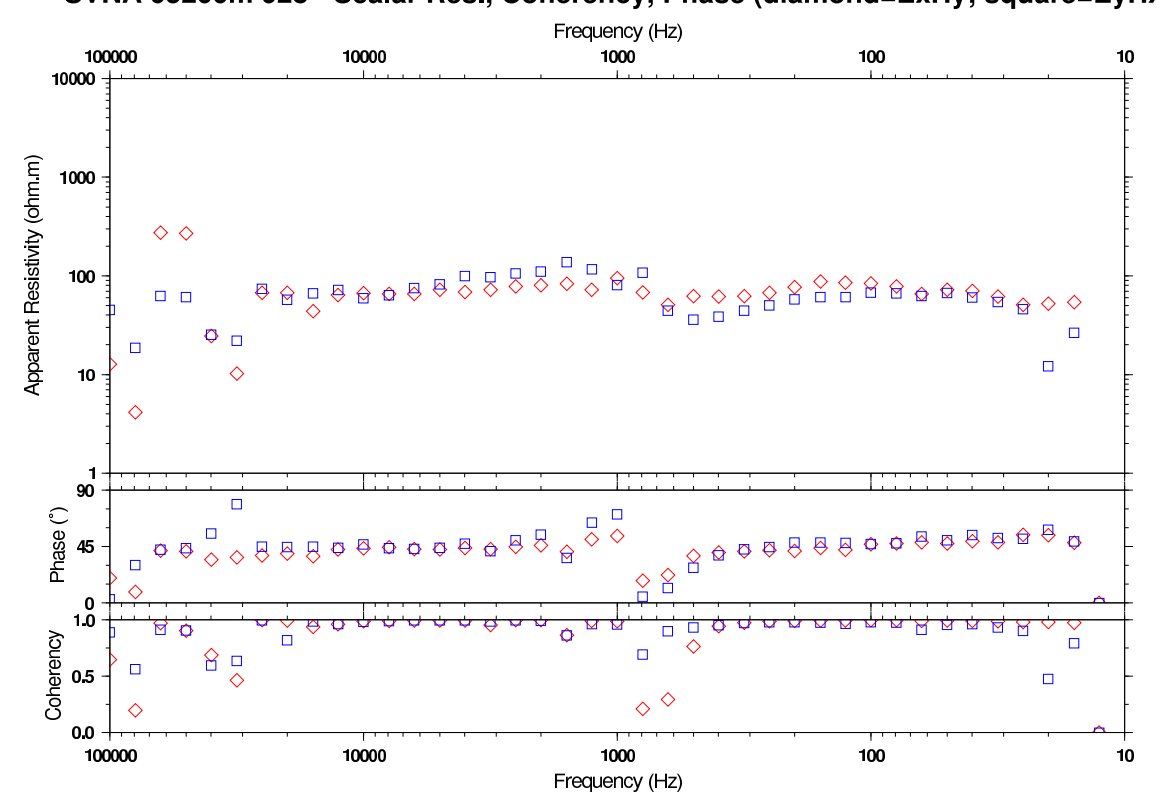

SVNA 05000m 063 - Scalar Res., Coherency, Phase (diamond=ExHy; square=EyHx)

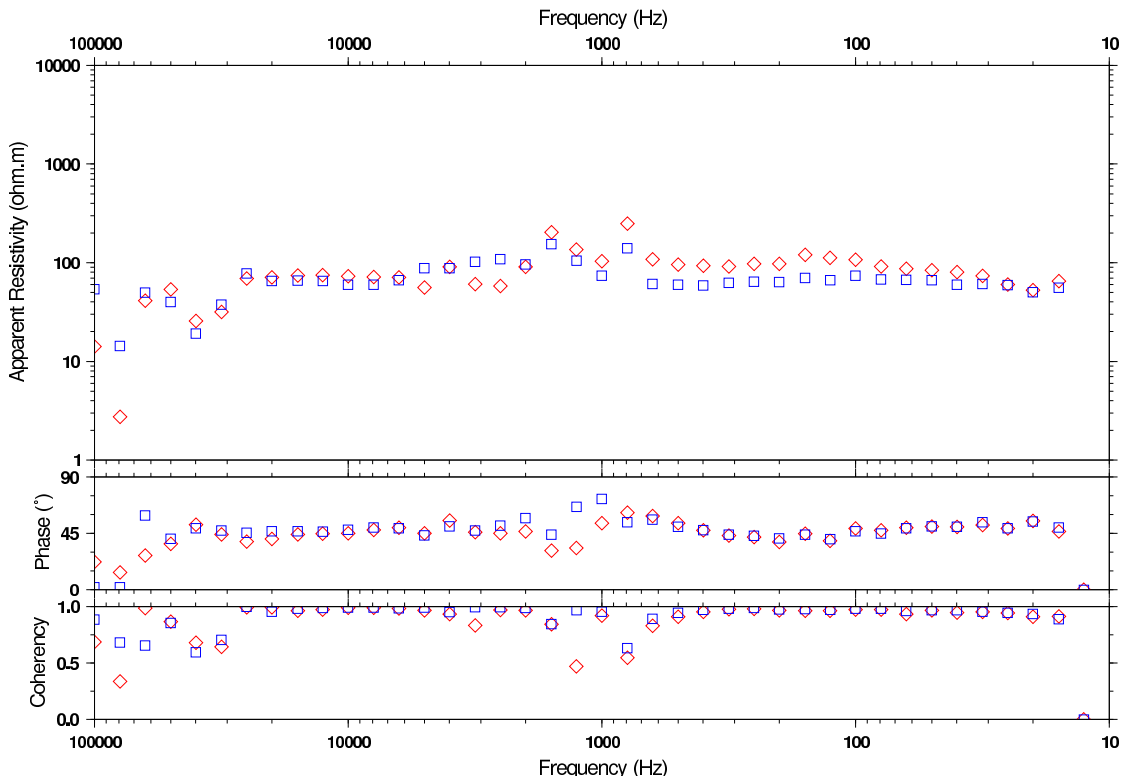

SVNA 05400m 064 - Scalar Res., Coherency, Phase (diamond=ExHy; square=EyHx)

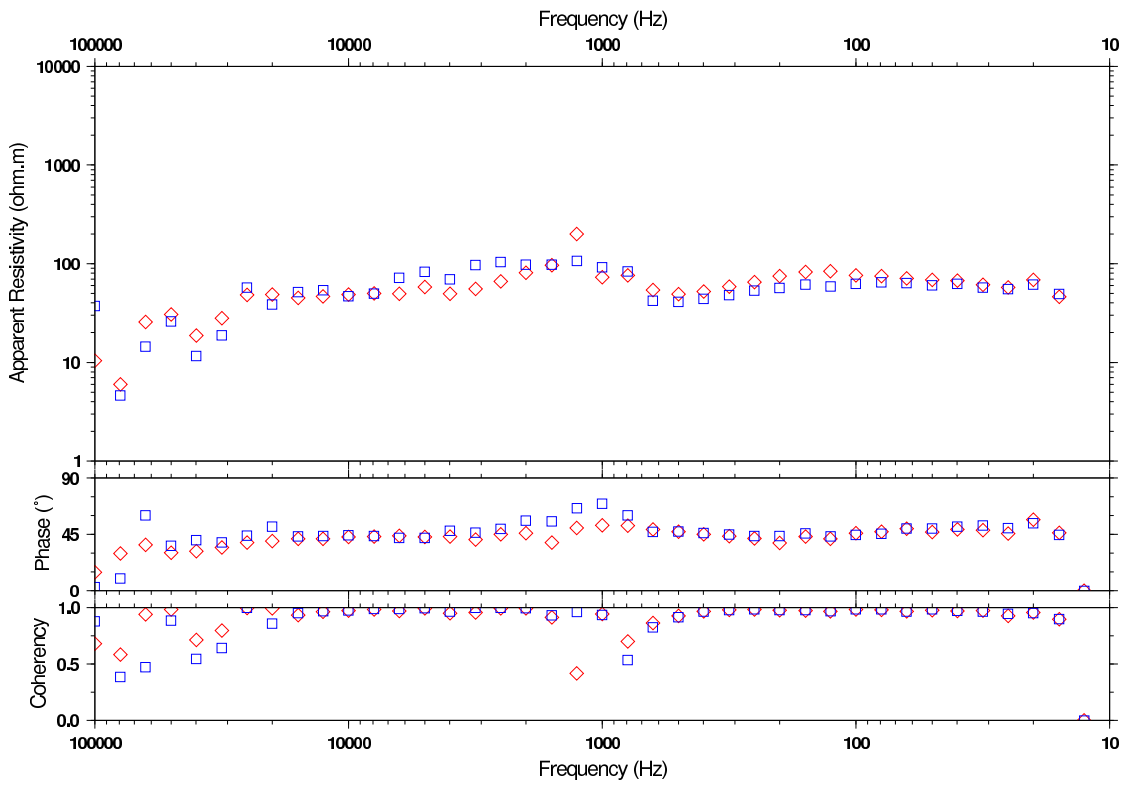


SVNA 05600m 030 - Scalar Res., Coherency, Phase (diamond=ExHy; square=EyHx)

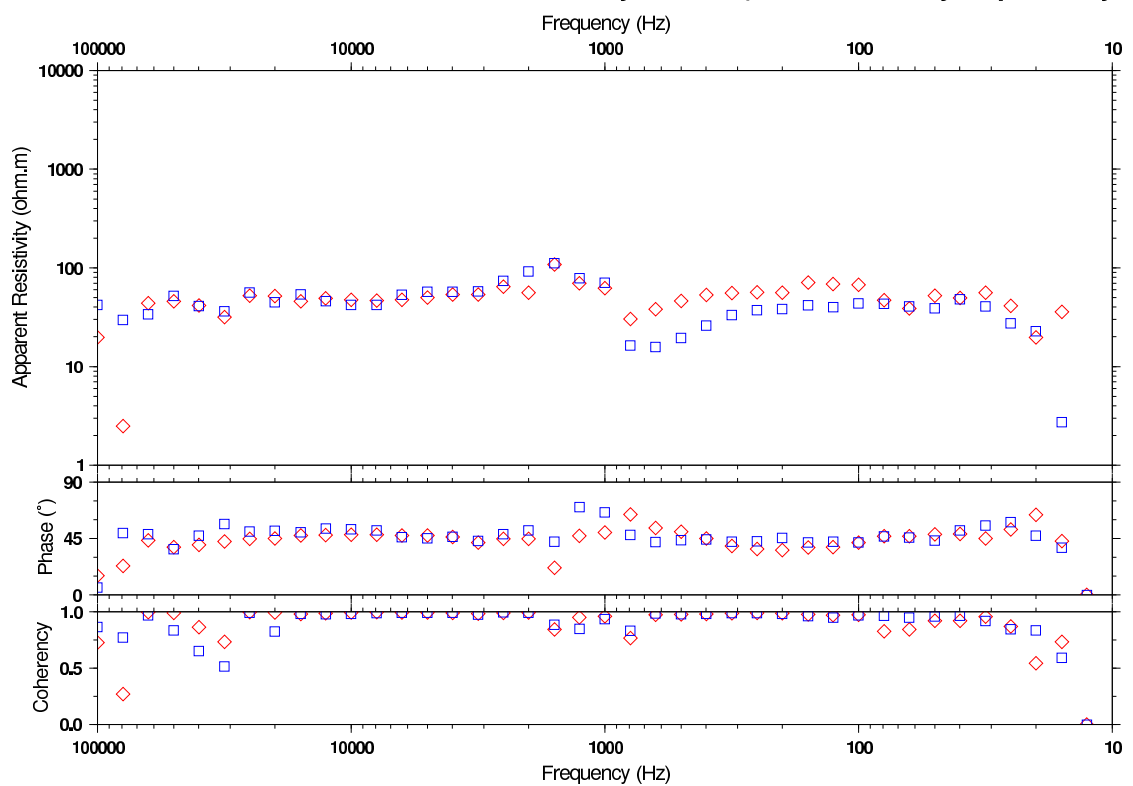

SVNA 05800m 065 - Scalar Res., Coherency, Phase (diamond=ExHy; square=EyHx)

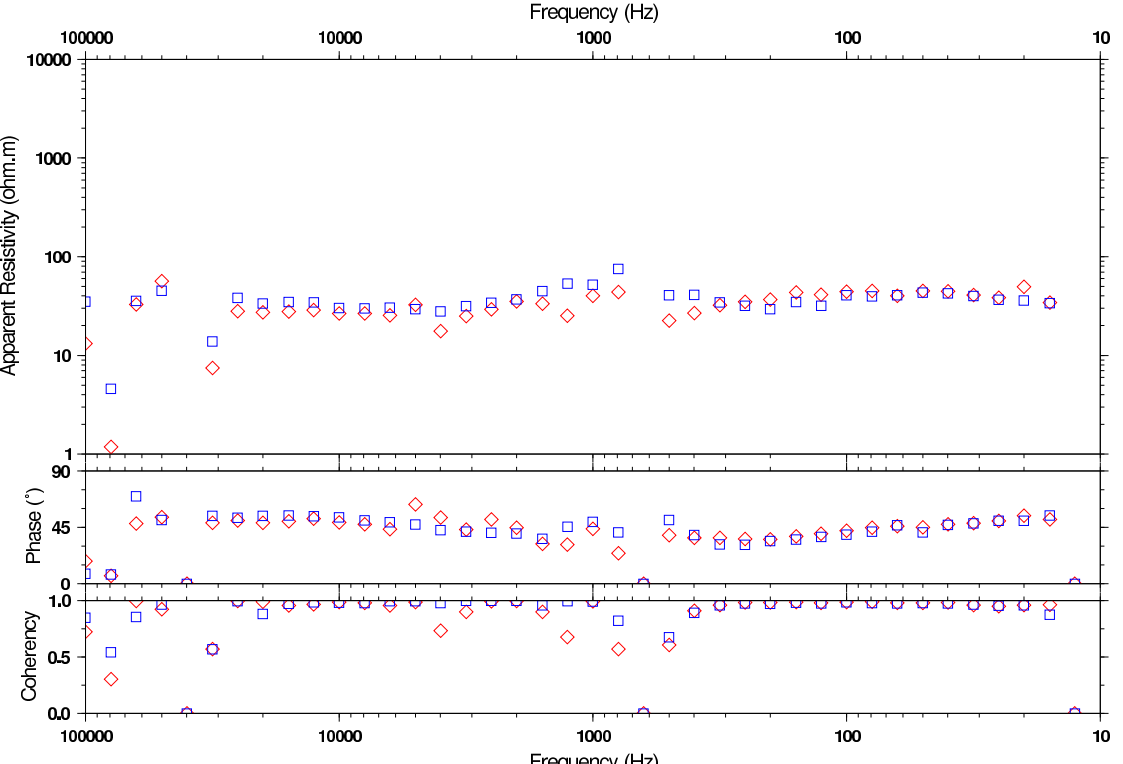

SVNA 06000m 032 - Scalar Res., Coherency, Phase (diamond=ExHy; square=EyHx)

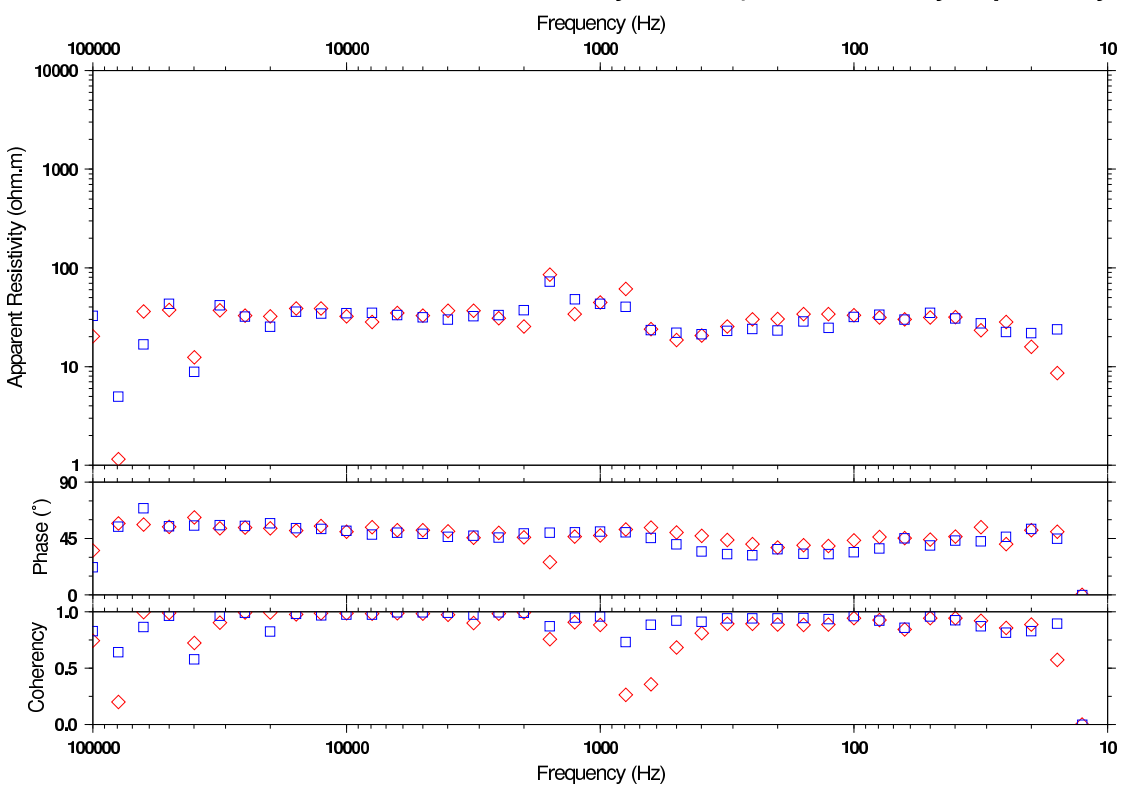

SVNA 06200m 067 - Scalar Res., Coherency, Phase (diamond=ExHy; square=EyHx)

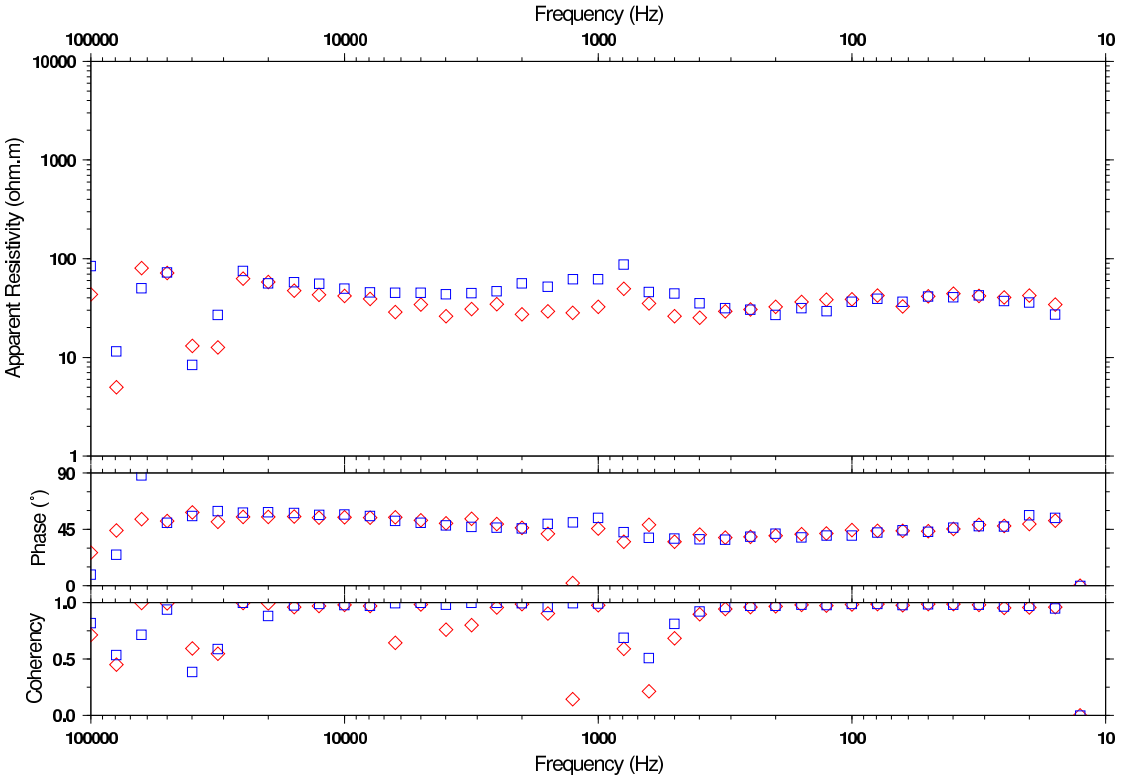


SVNA 06400m 034 - Scalar Res., Coherency, Phase (diamond=ExHy; square=EyHx)

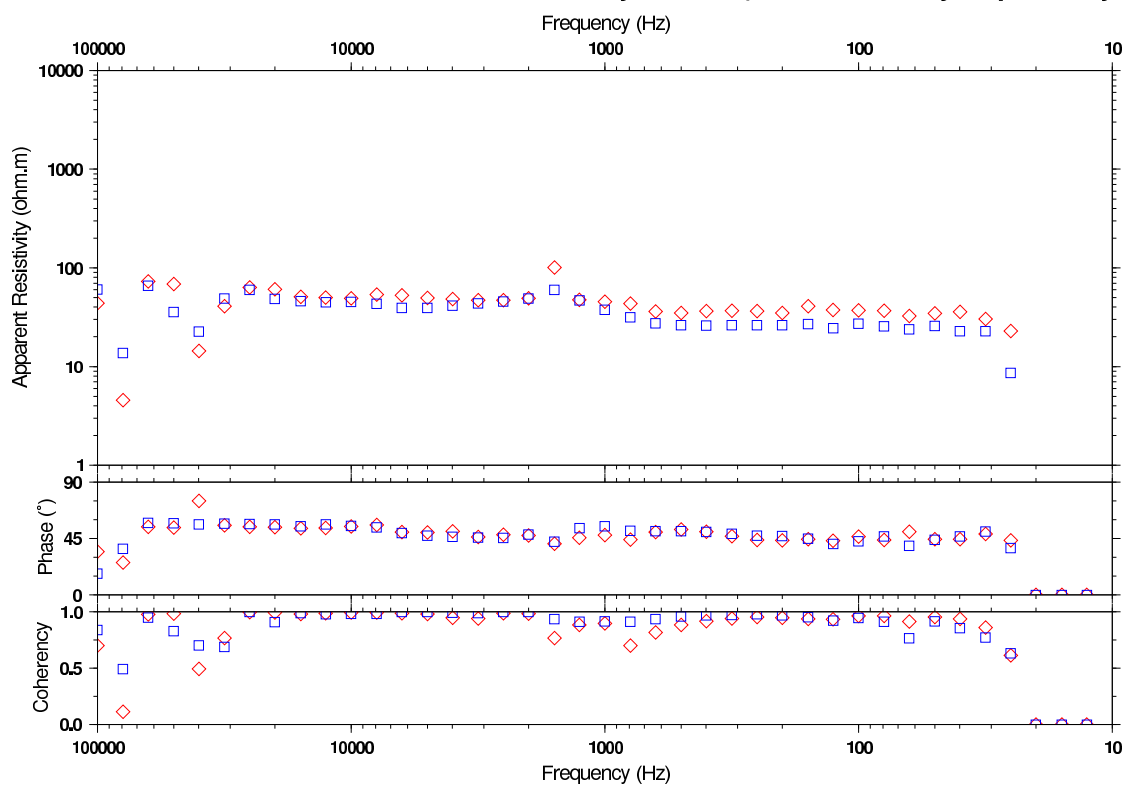

SVNA 06600m 068 - Scalar Res., Coherency, Phase (diamond=ExHy; square=EyHx)

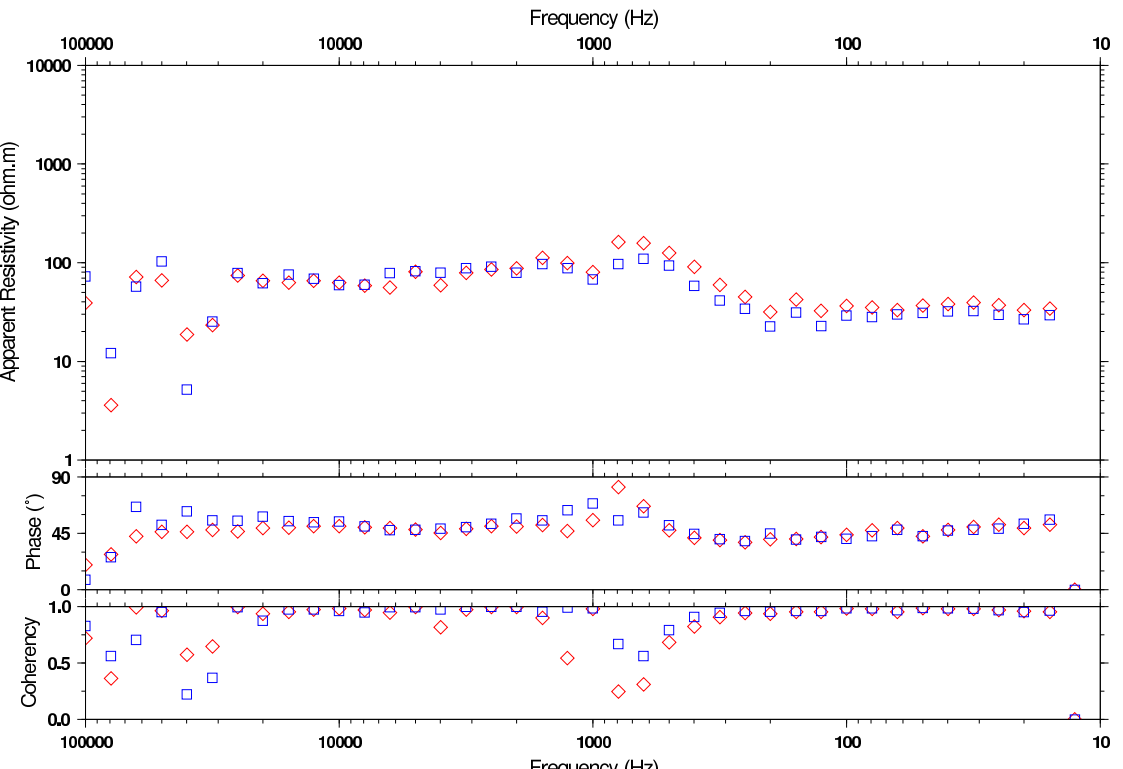

SVNA 06800m 035 - Scalar Res., Coherency, Phase (diamond=ExHy; square=EyHx)

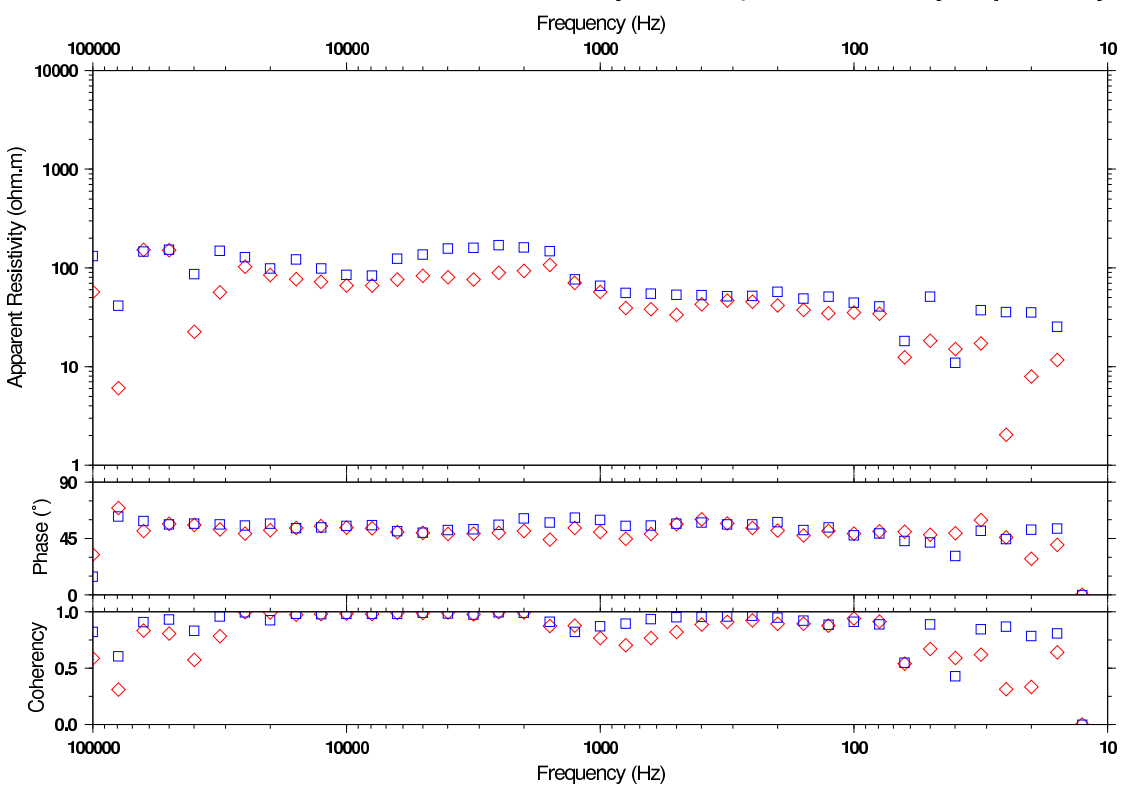

SVNA 07000m 069 - Scalar Res., Coherency, Phase (diamond=ExHy; square=EyHx)

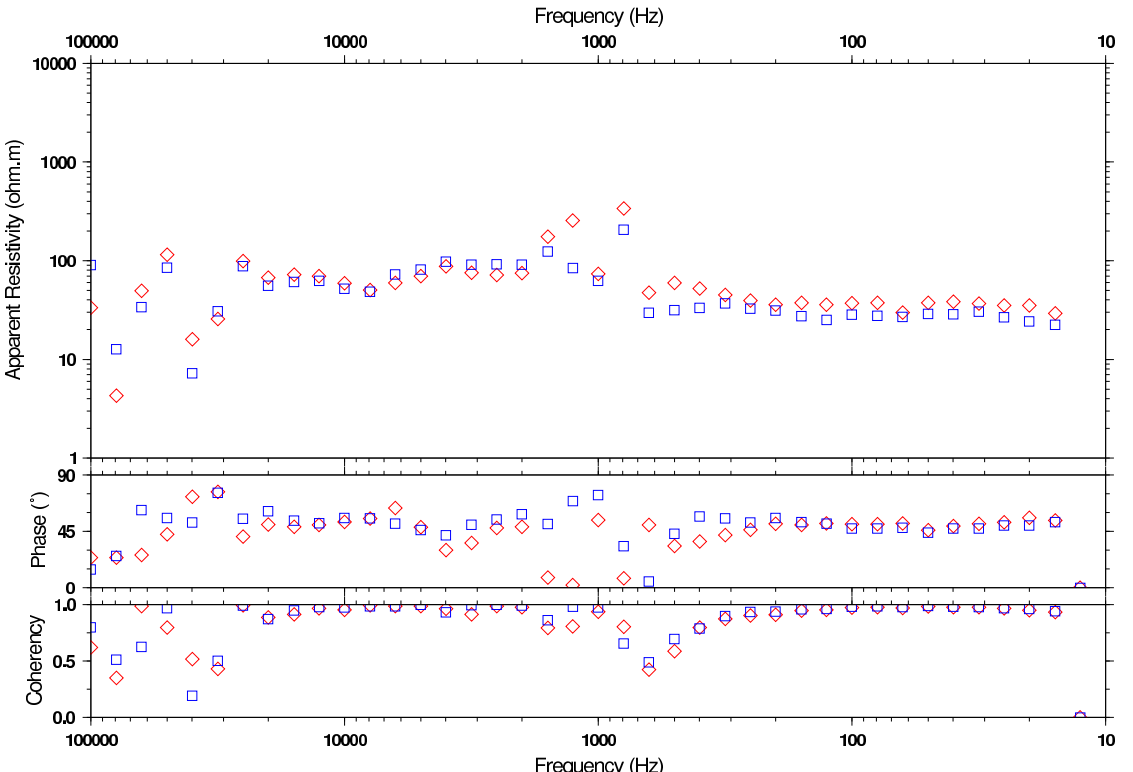




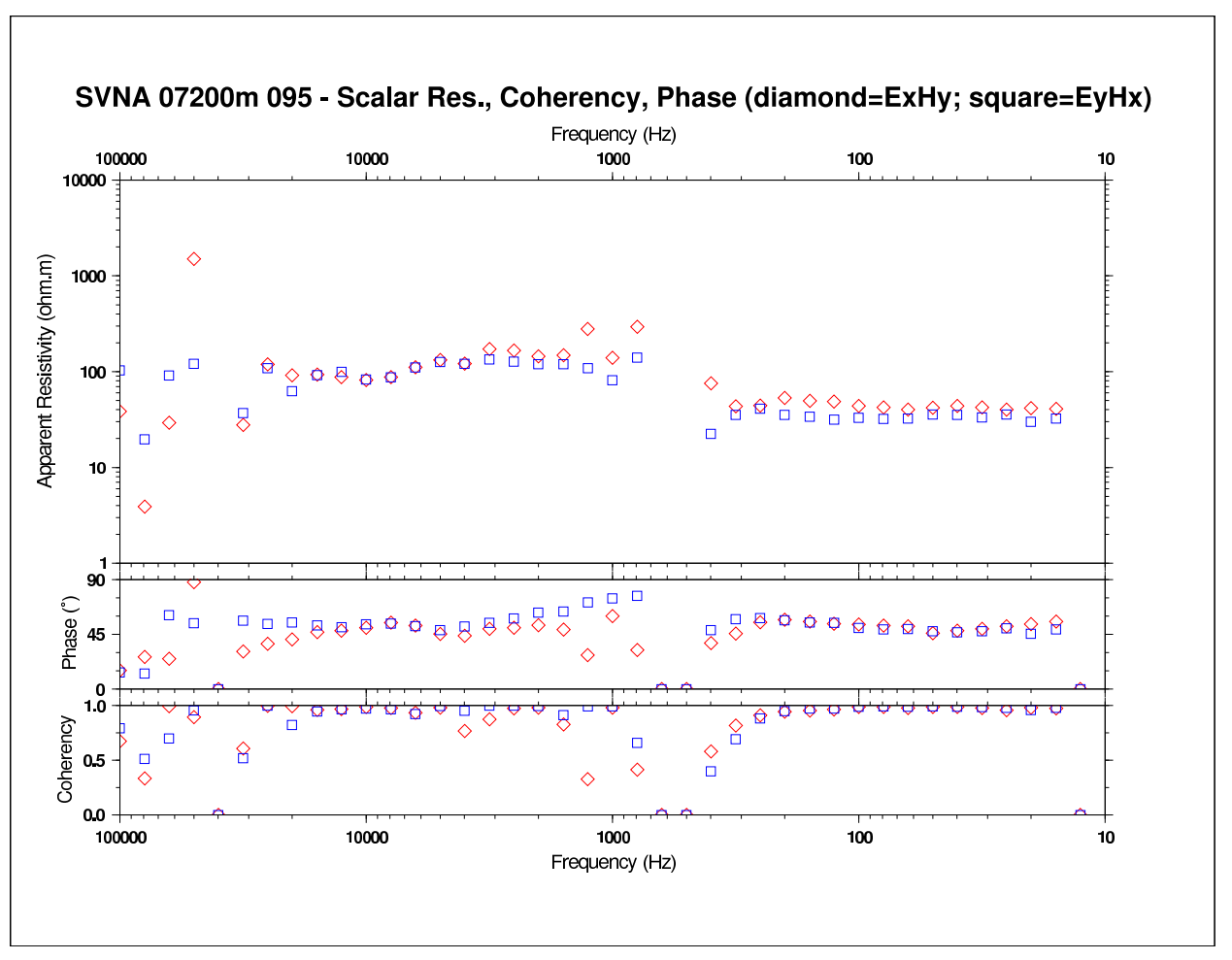

SVNA 07600m 039 - Scalar Res., Coherency, Phase (diamond=ExHy; square=EyHx)

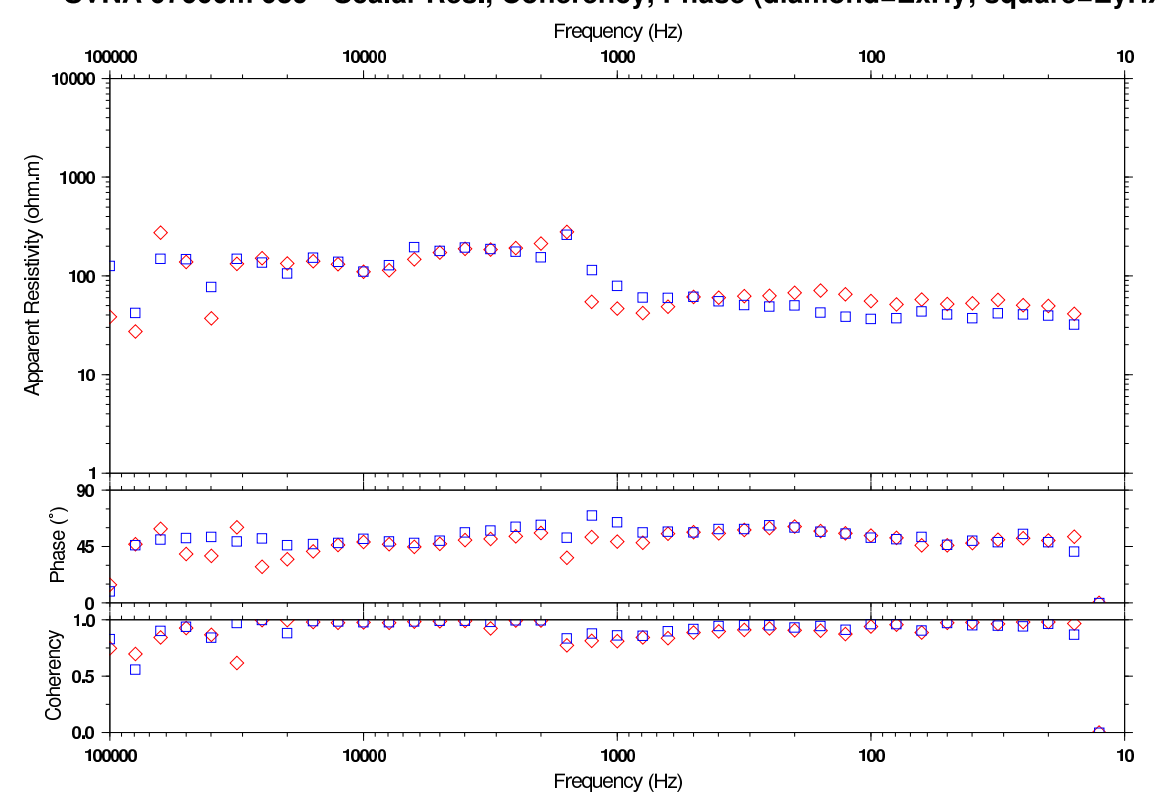

SVNA 07400m 070 - Scalar Res., Coherency, Phase (diamond=ExHy; square=EyHx)

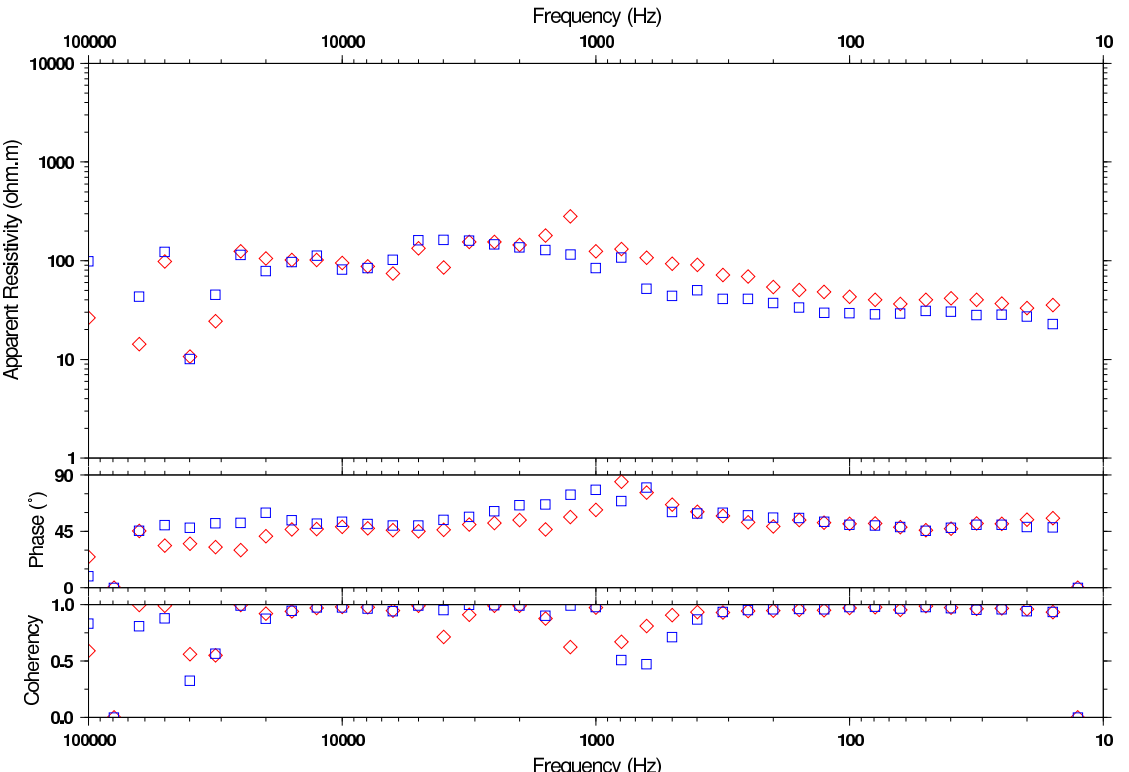

SVNA 07800m 071 - Scalar Res., Coherency, Phase (diamond=ExHy; square=EyHx)

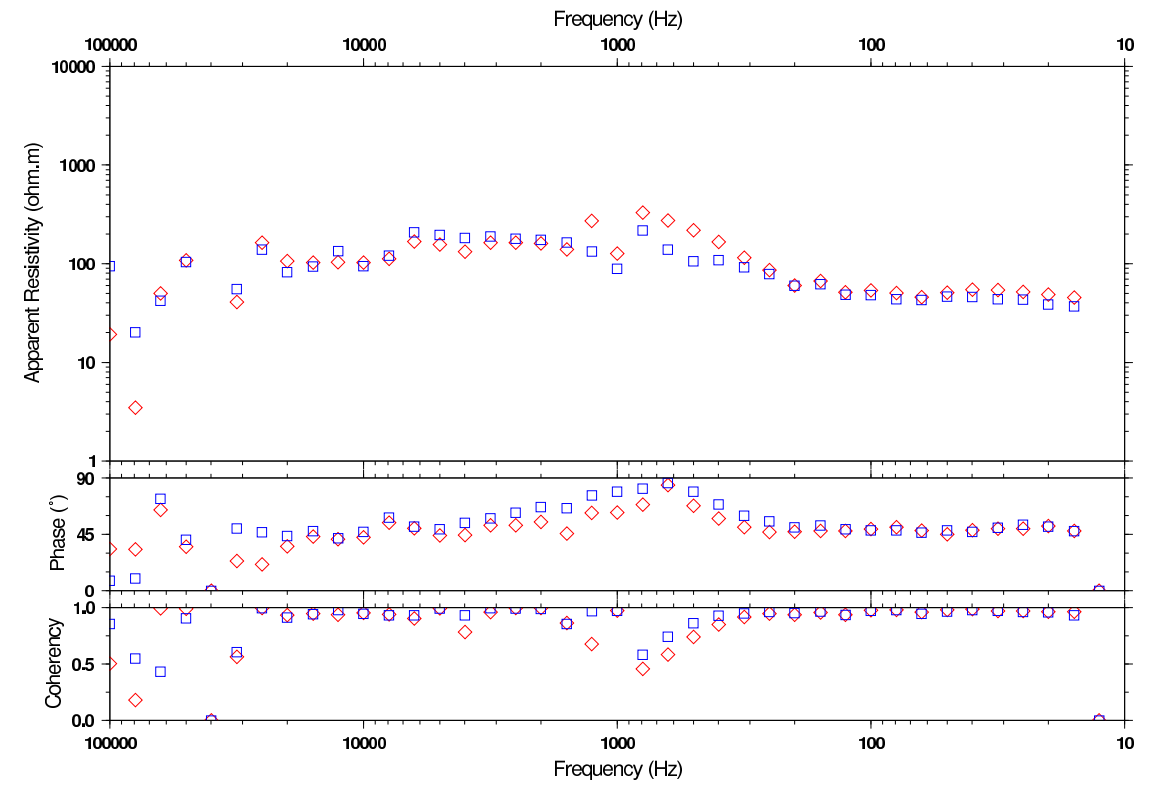


SVNA 08000m 040 - Scalar Res., Coherency, Phase (diamond=ExHy; square=EyHx)

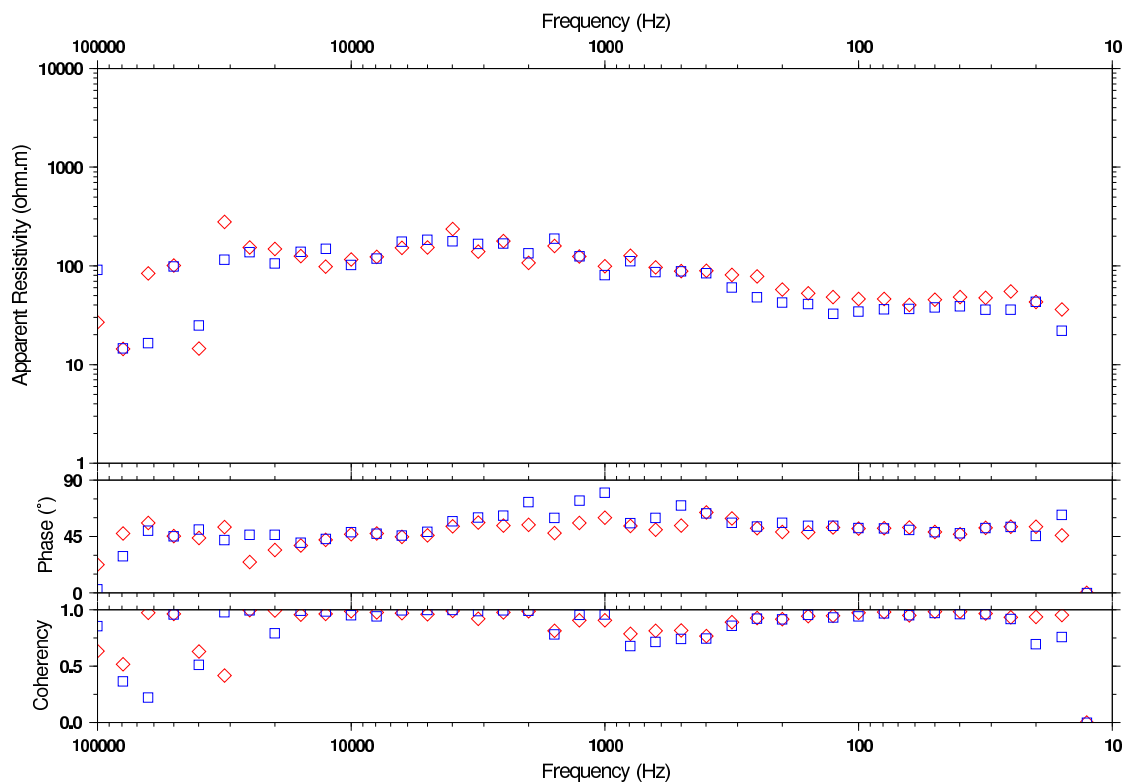

SVNA 08200m 072 - Scalar Res., Coherency, Phase (diamond=ExHy; square=EyHx)

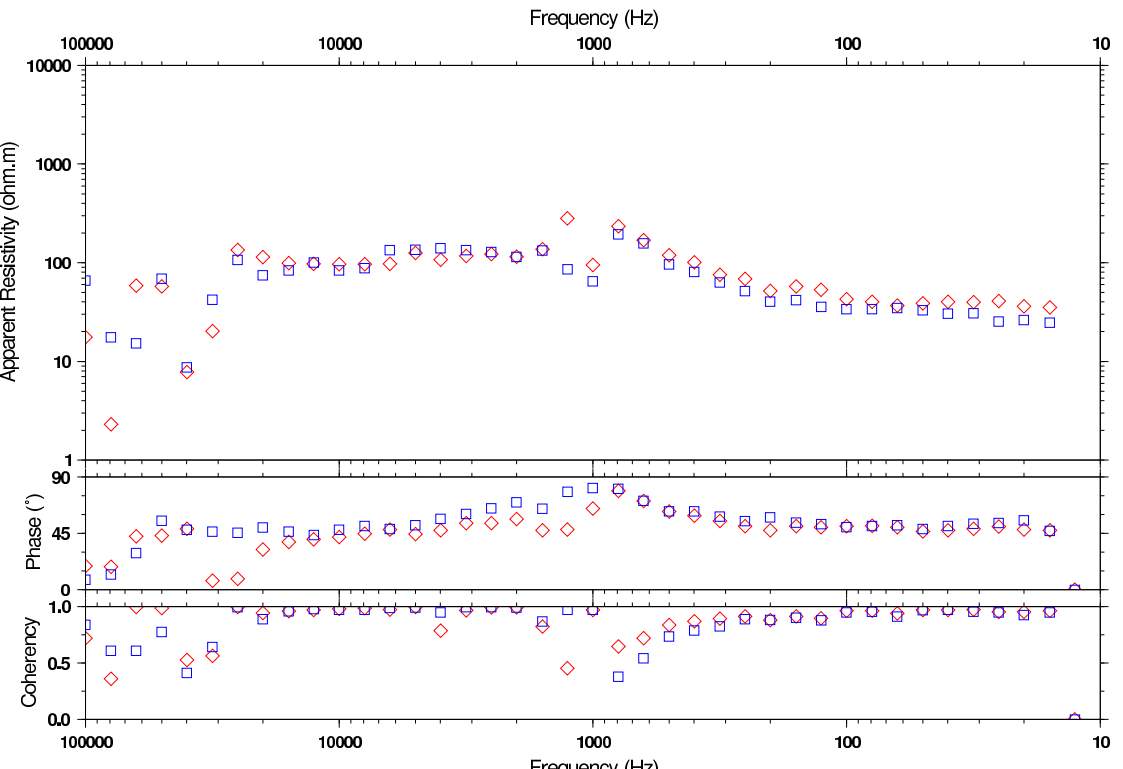

SVNA 08400m 041 - Scalar Res., Coherency, Phase (diamond=ExHy; square=EyHx)

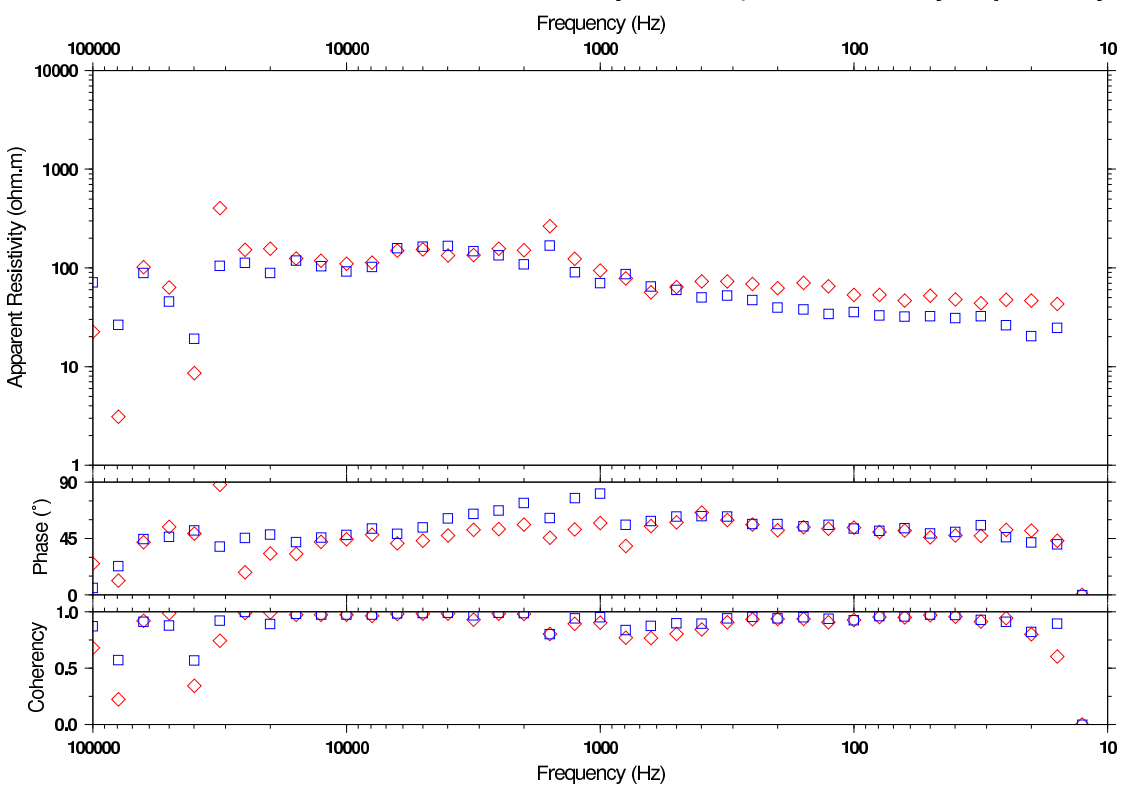

SVNA 08600m 074 - Scalar Res., Coherency, Phase (diamond=ExHy; square=EyHx)

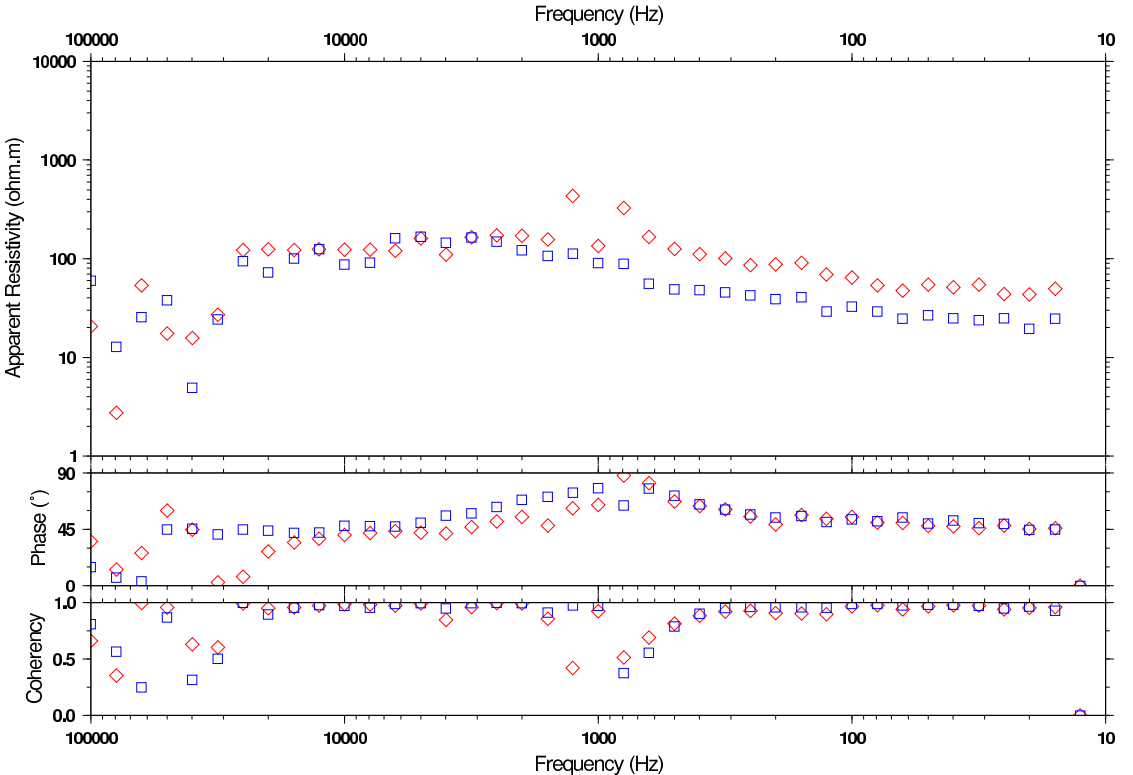


SVNA 08800m 043 - Scalar Res., Coherency, Phase (diamond=ExHy; square=EyHx)

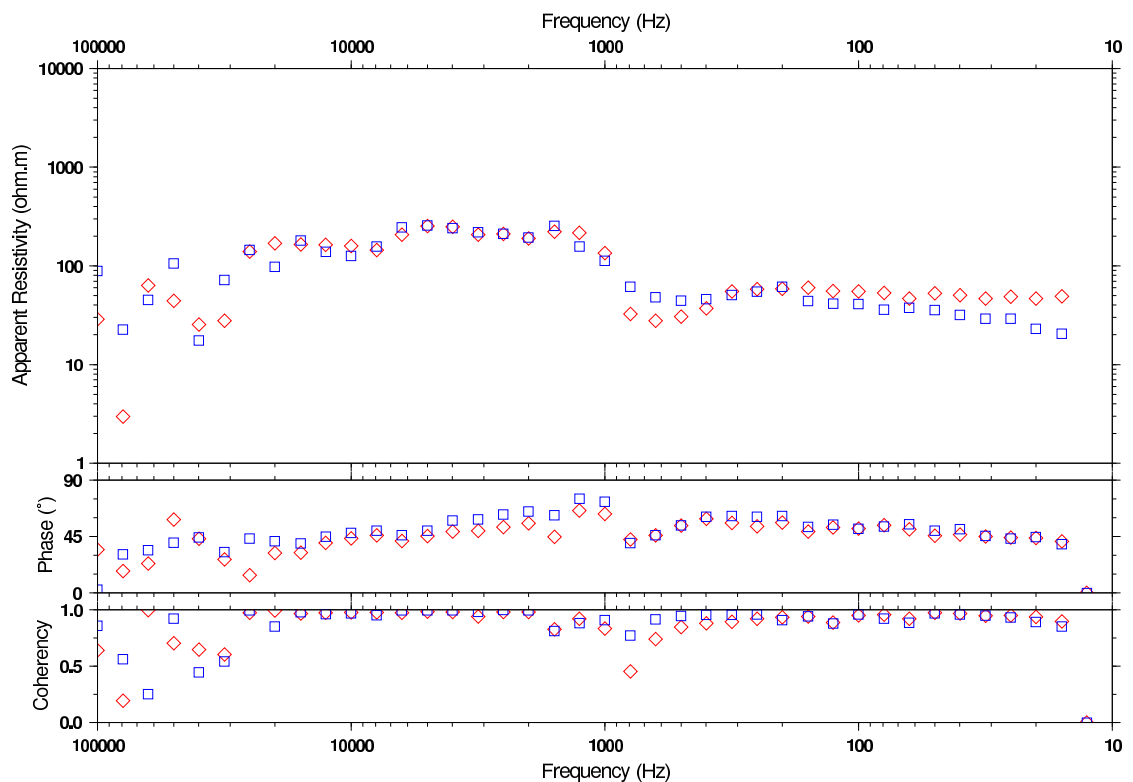

SVNA 09000m 075 - Scalar Res., Coherency, Phase (diamond=ExHy; square=EyHx)

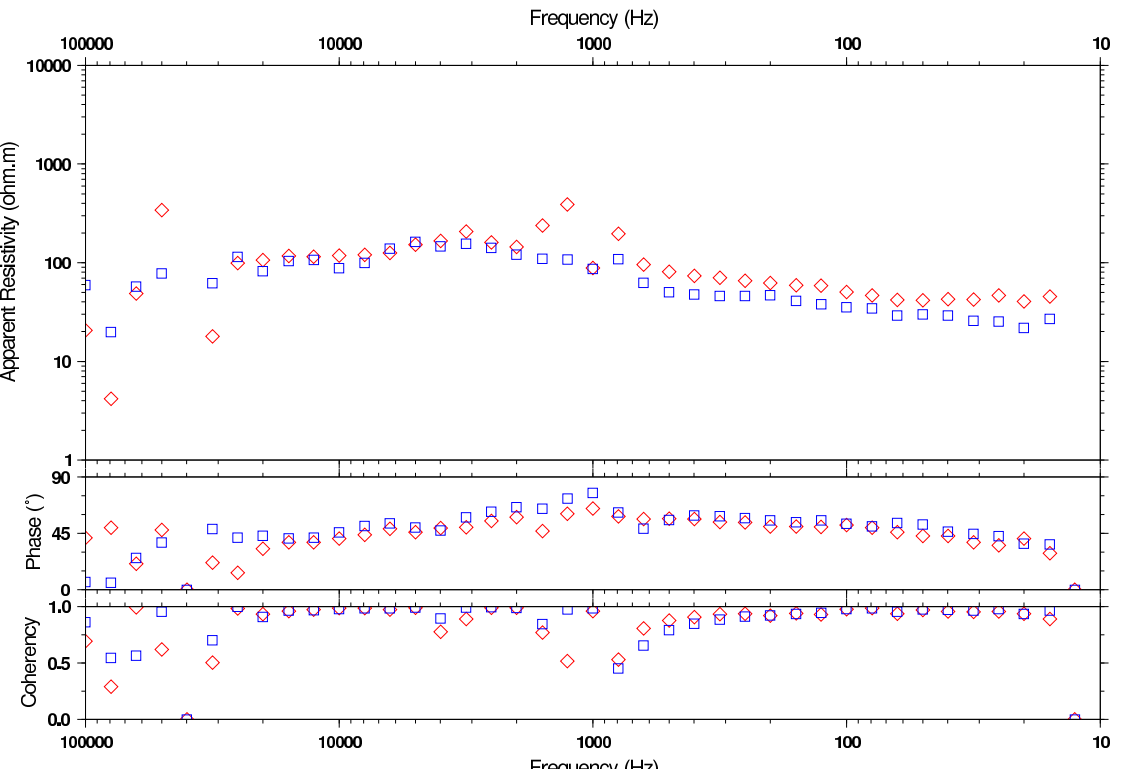

SVNA 09200m 045 - Scalar Res., Coherency, Phase (diamond=ExHy; square=EyHx)

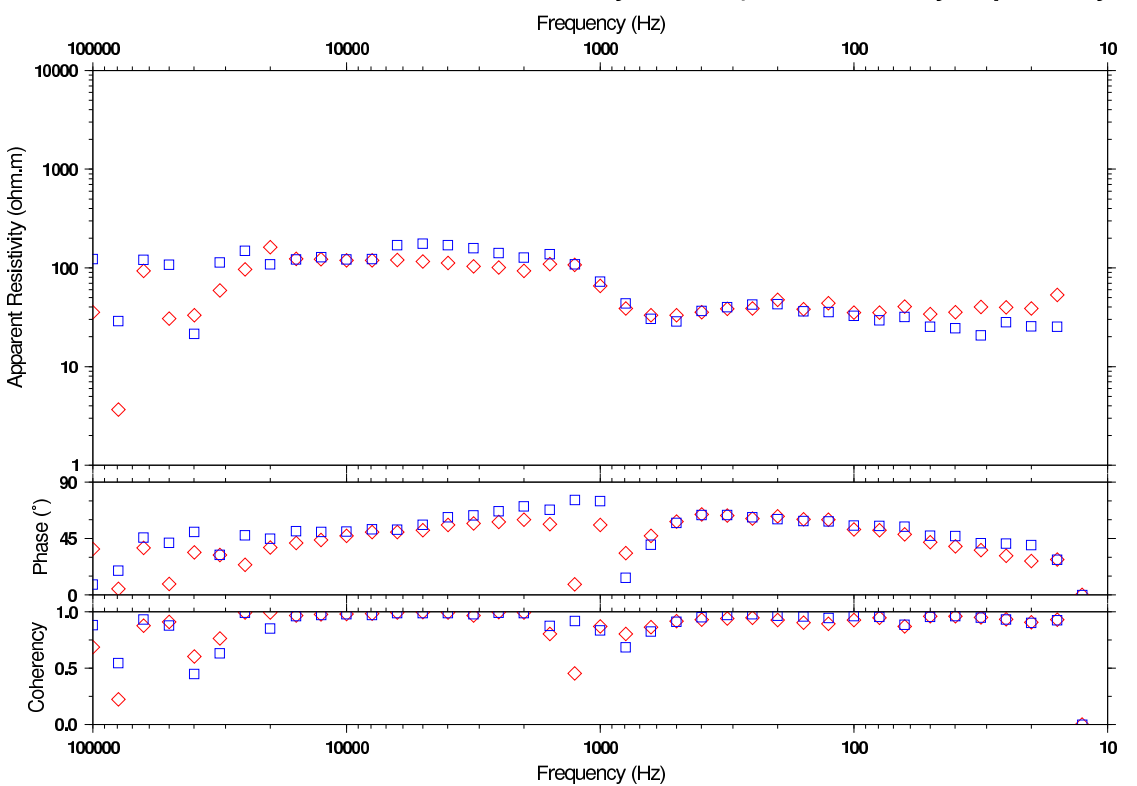

SVNA 09400m 077 - Scalar Res., Coherency, Phase (diamond=ExHy; square=EyHx)

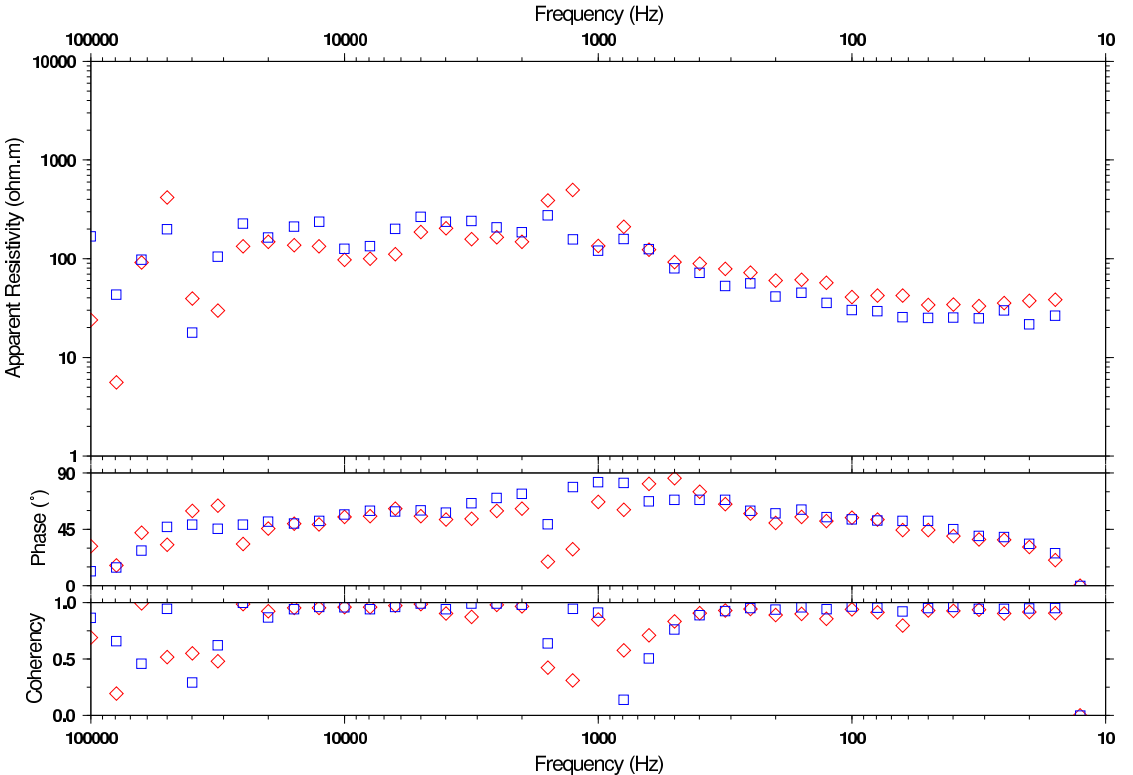


SVNA 09600m 046 - Scalar Res., Coherency, Phase (diamond=ExHy; square=EyHx)

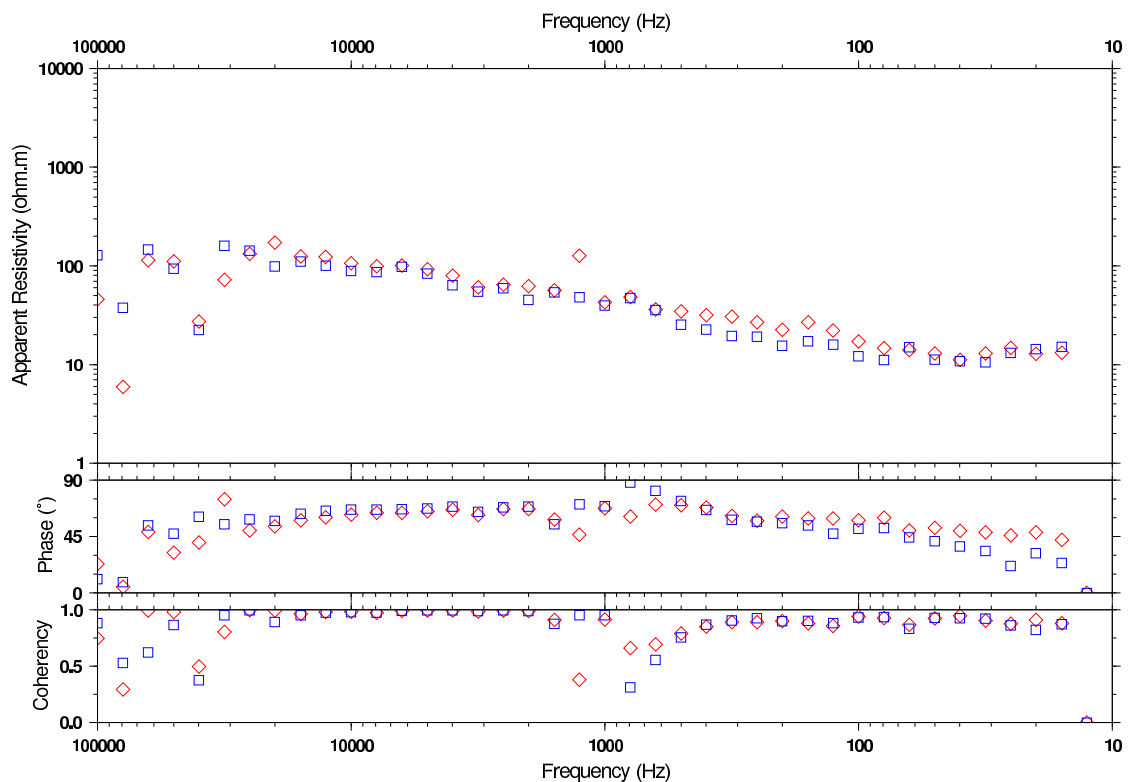

SVNA 09800m 078 - Scalar Res., Coherency, Phase (diamond=ExHy; square=EyHx)

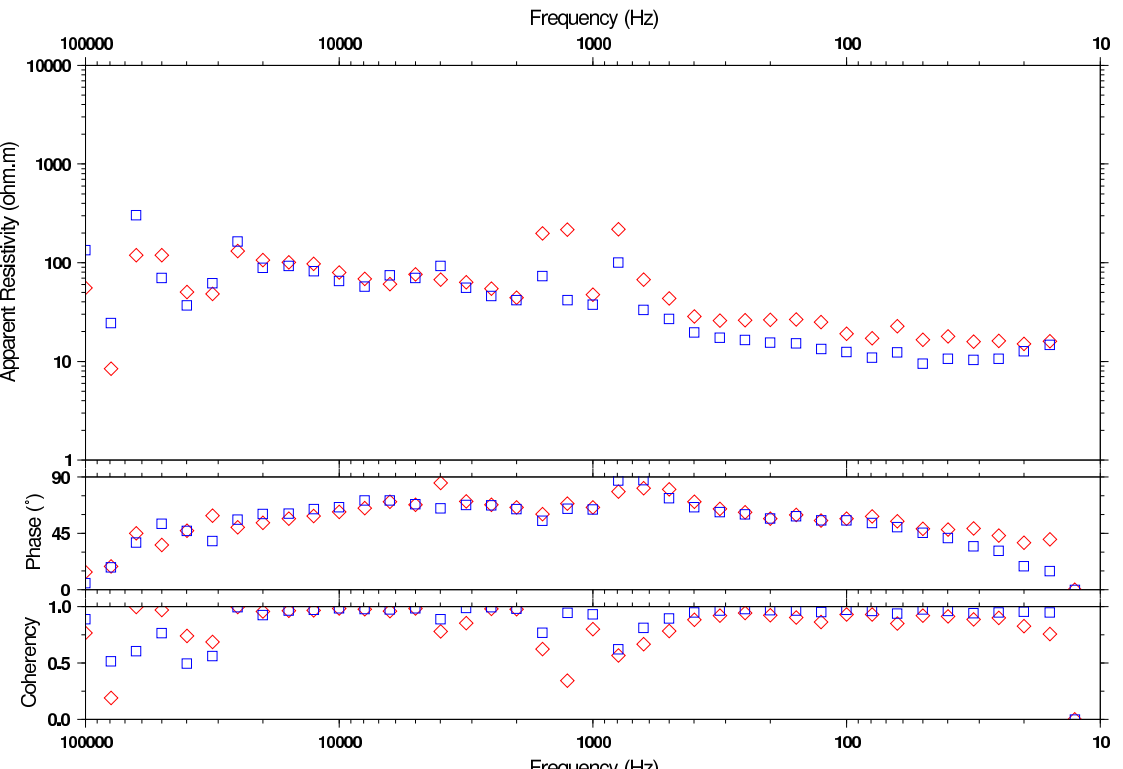

SVNA 10000m 047 - Scalar Res., Coherency, Phase (diamond=ExHy; square=EyHx)

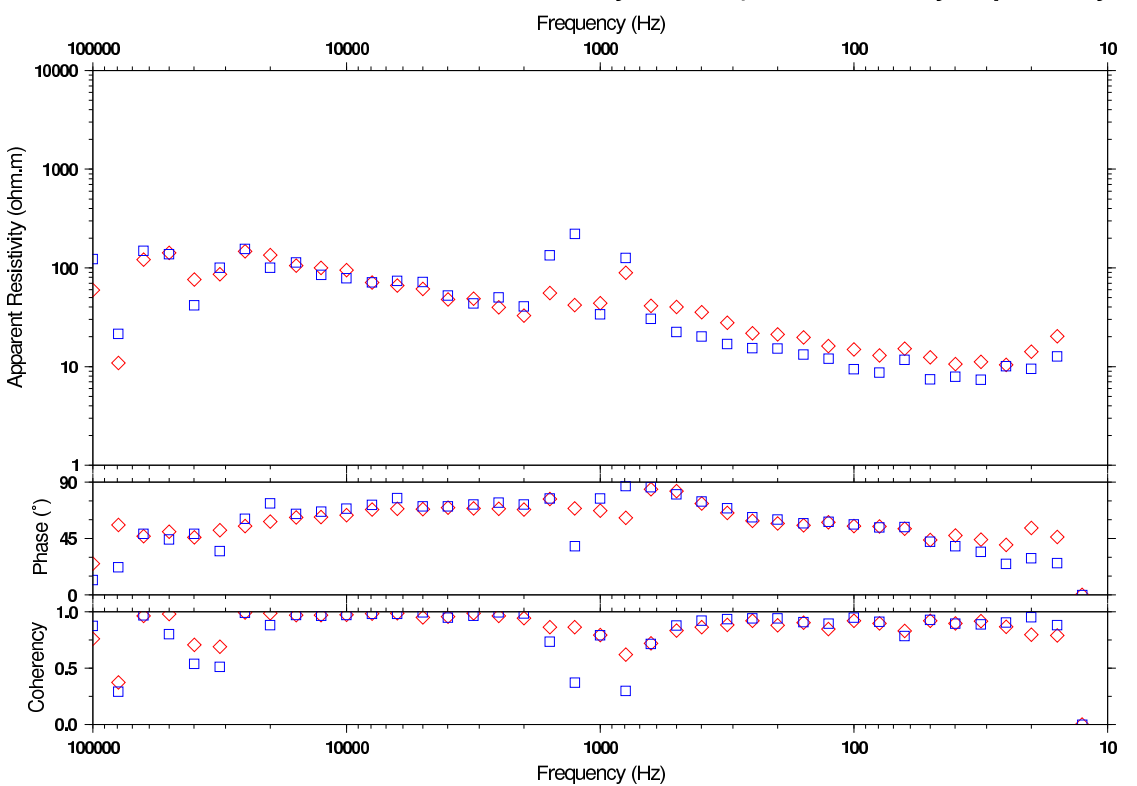

SVNA 10200m 080 - Scalar Res., Coherency, Phase (diamond=ExHy; square=EyHx)

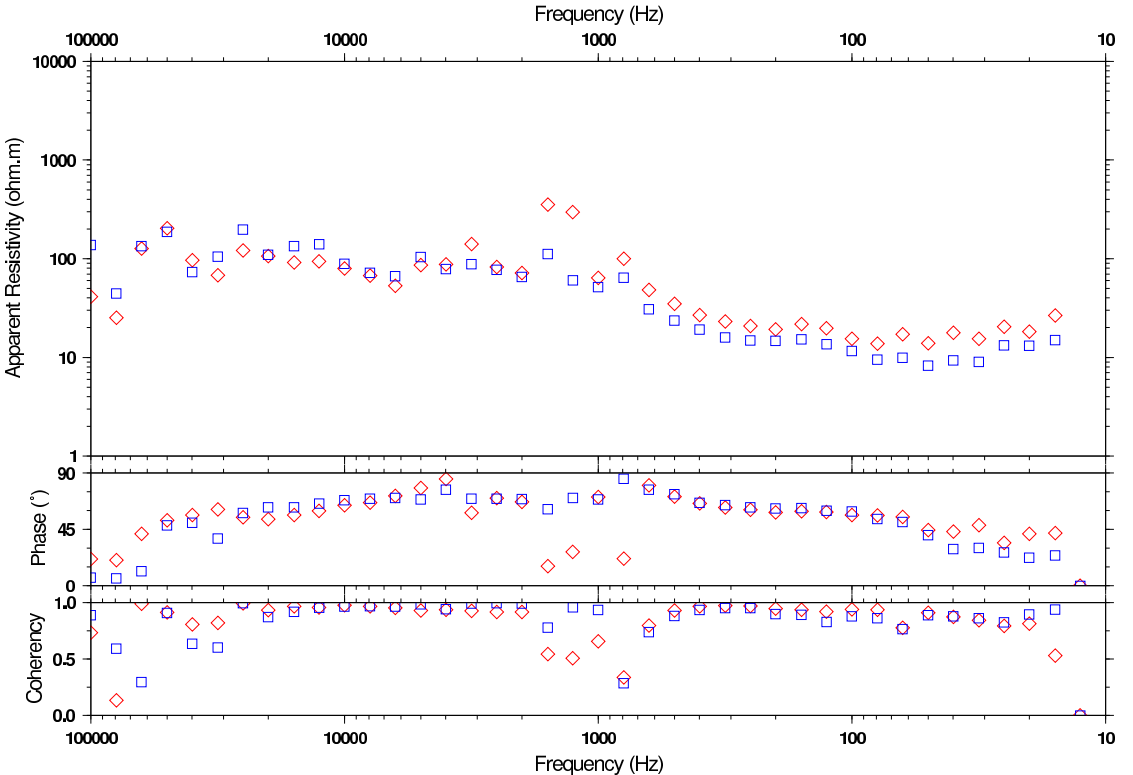


SVNA 10400m 049 - Scalar Res., Coherency, Phase (diamond=ExHy; square=EyHx)

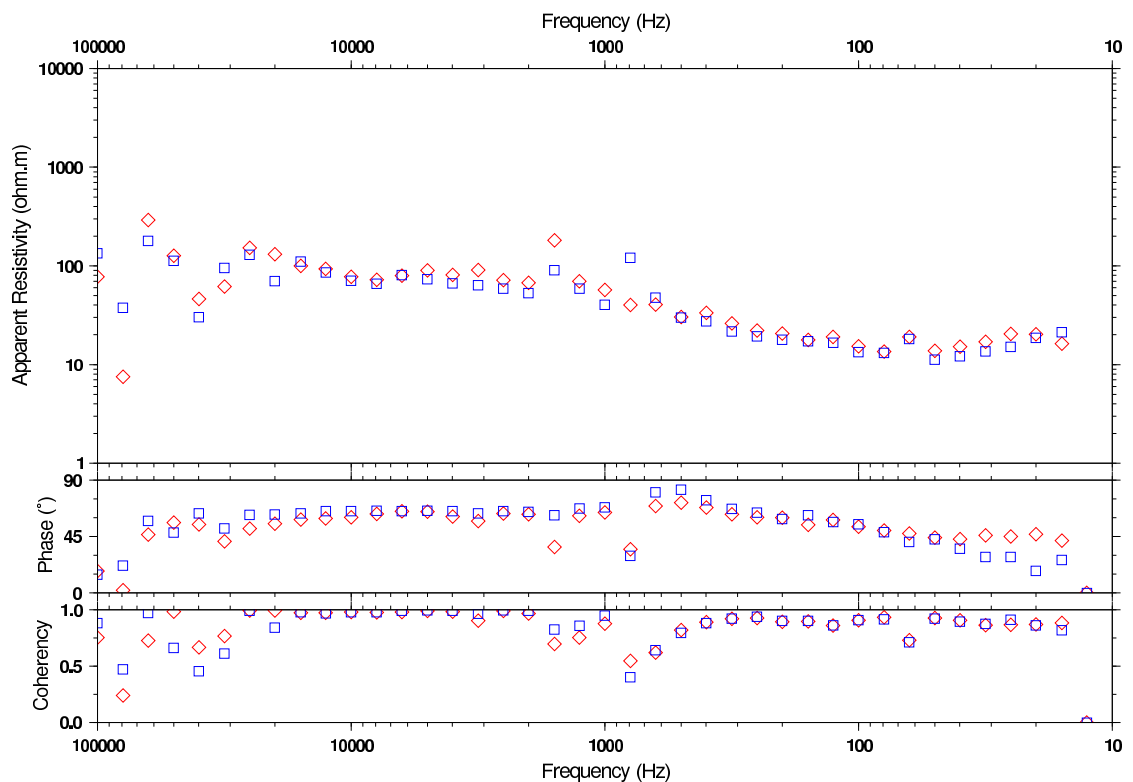

SVNA 10600m 082 - Scalar Res., Coherency, Phase (diamond=ExHy; square=EyHx)

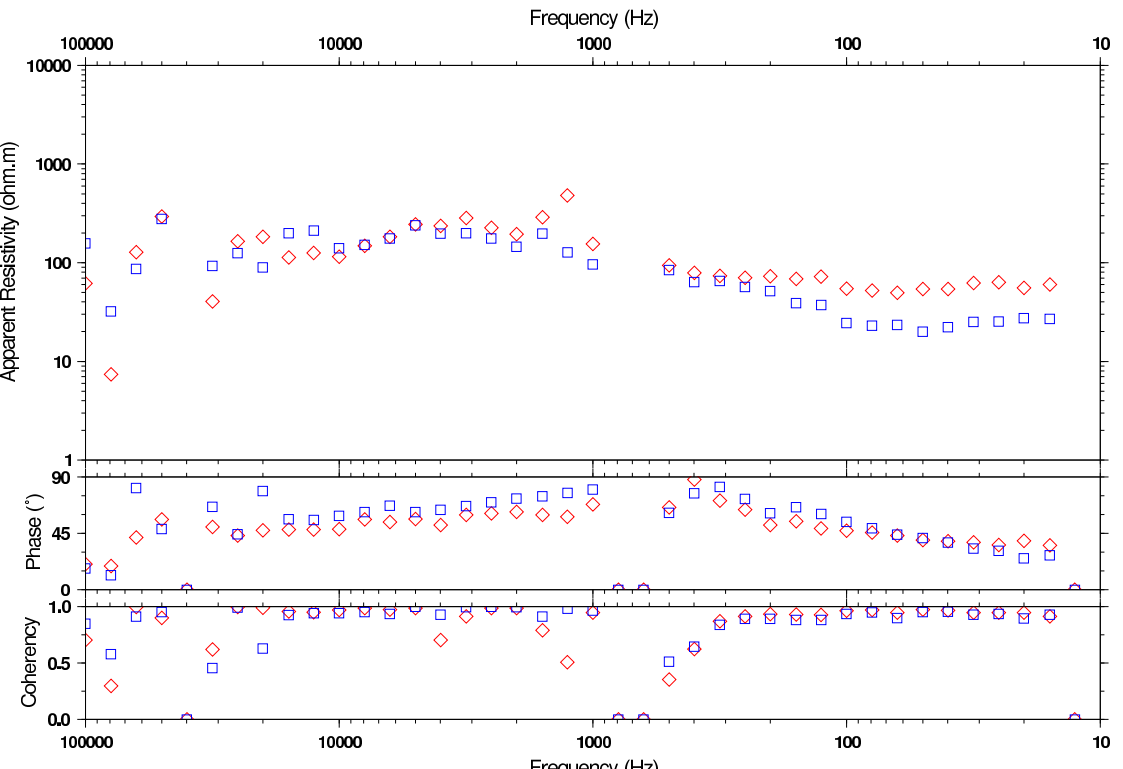

SVNA 10800m 051 - Scalar Res., Coherency, Phase (diamond=ExHy; square=EyHx)

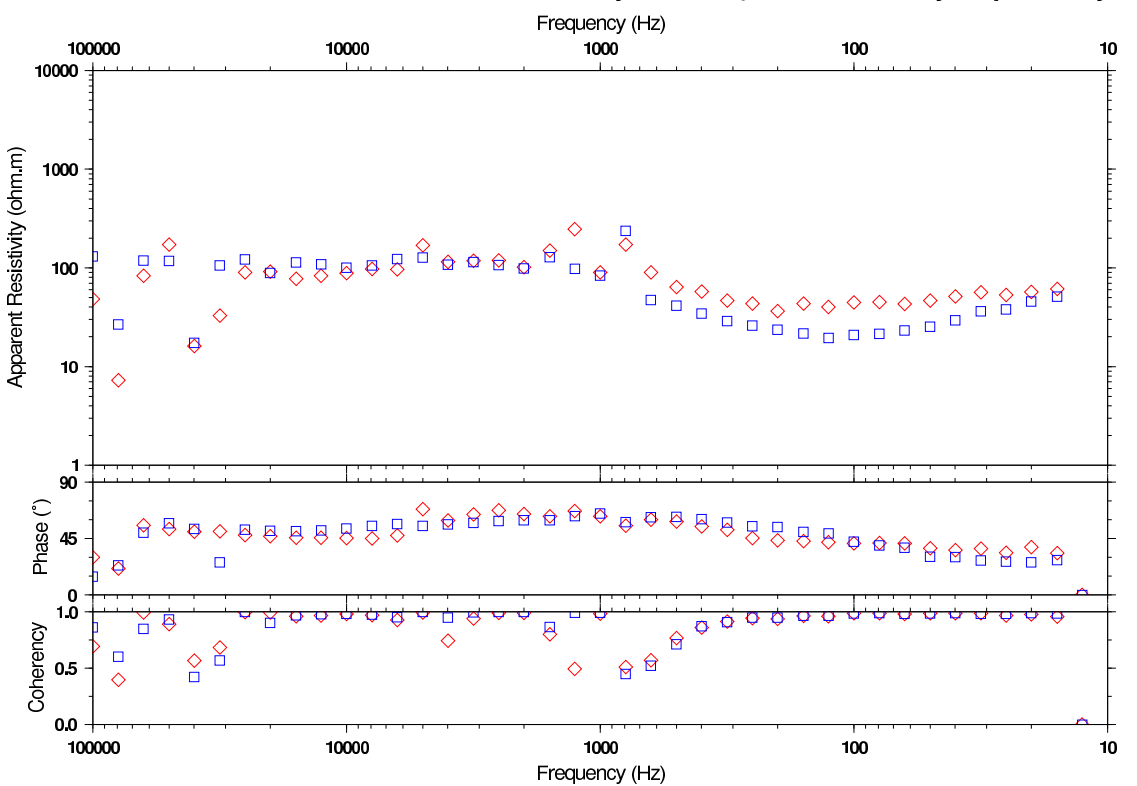

SVNA 11000m 083 - Scalar Res., Coherency, Phase (diamond=ExHy; square=EyHx)

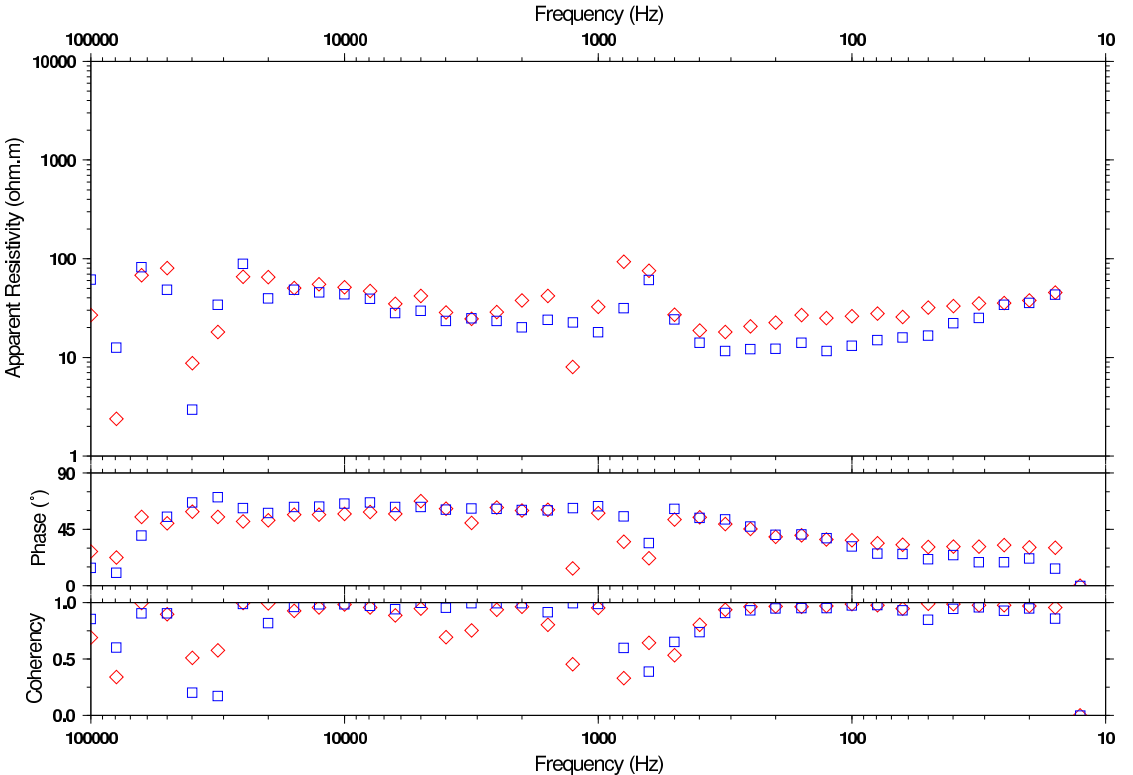


SVNA 11200m 052 - Scalar Res., Coherency, Phase (diamond=ExHy; square=EyHx)

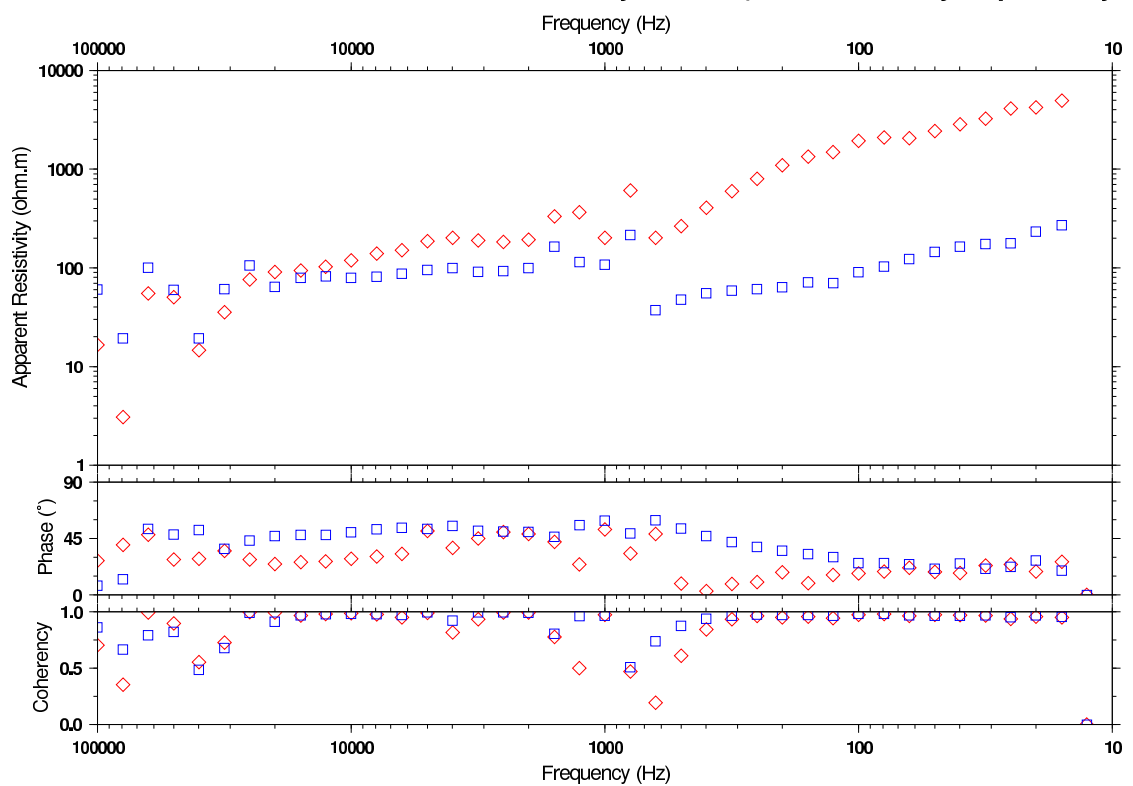

SVNA 11400m 084 - Scalar Res., Coherency, Phase (diamond=ExHy; square=EyHx)

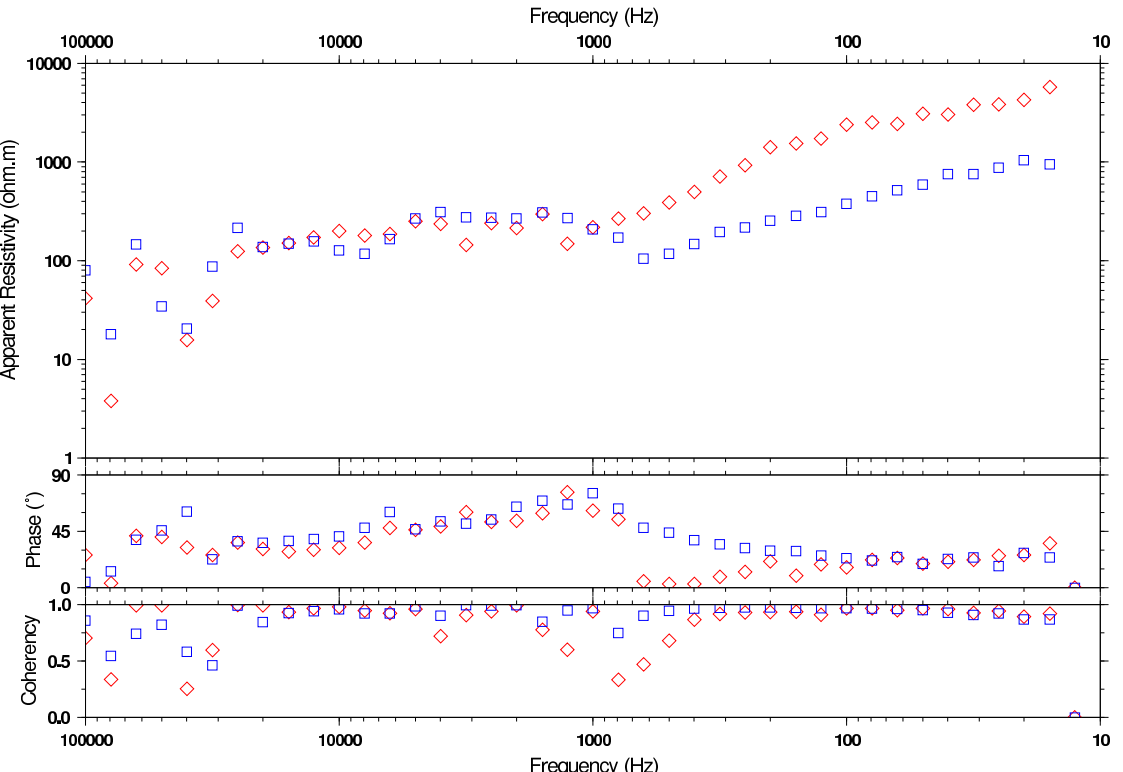

SVNA 11600m 098 - Scalar Res., Coherency, Phase (diamond=ExHy; square=EyHx)

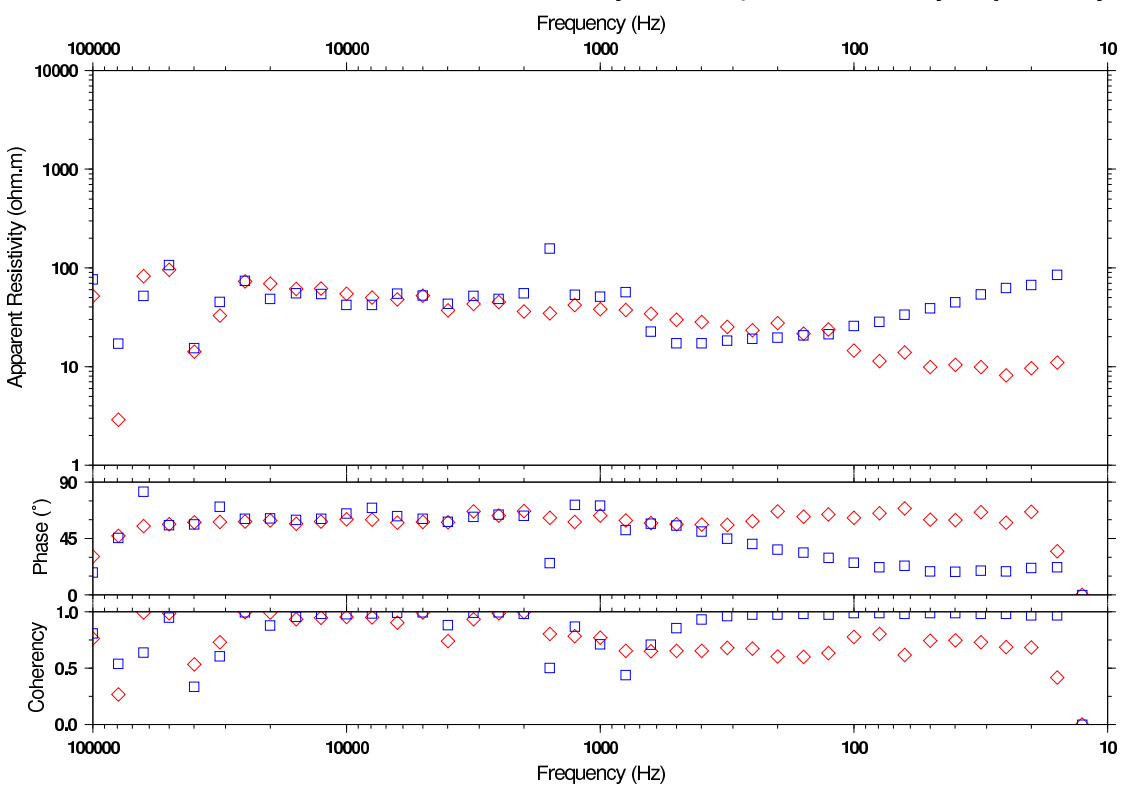

SVNA 11800m 087 - Scalar Res., Coherency, Phase (diamond=ExHy; square=EyHx)

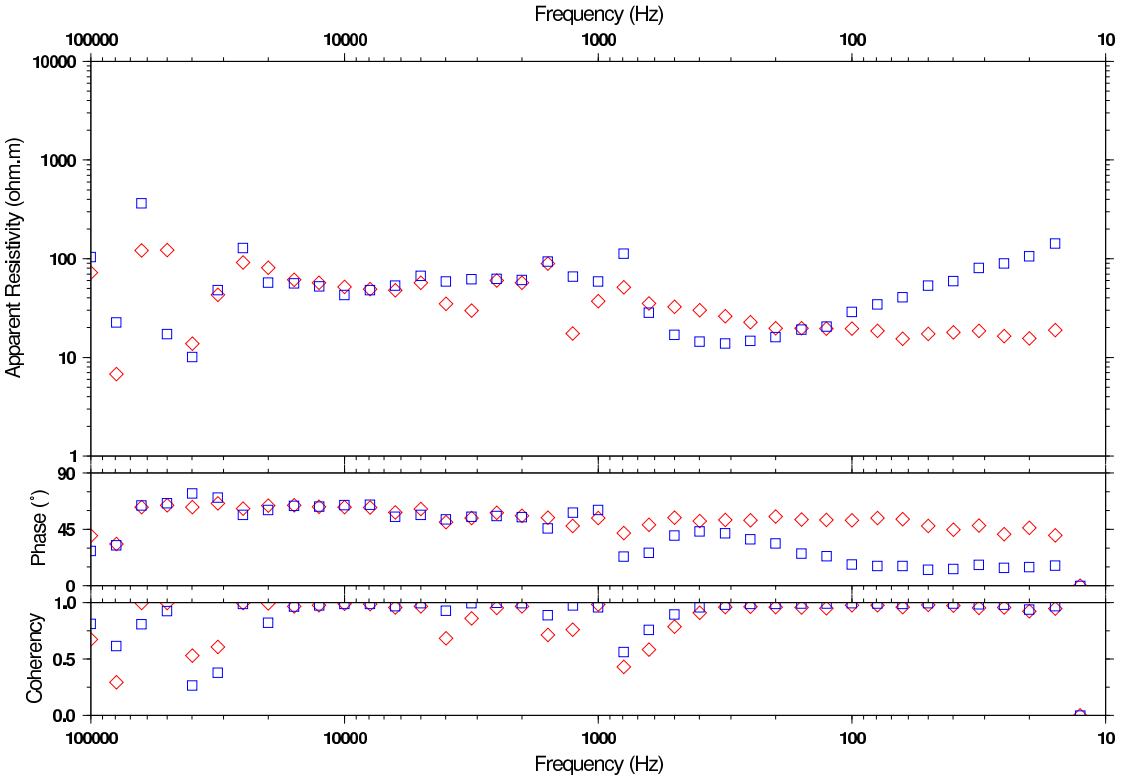


SVNA 12000m 094 - Scalar Res., Coherency, Phase (diamond=ExHy; square=EyHx)

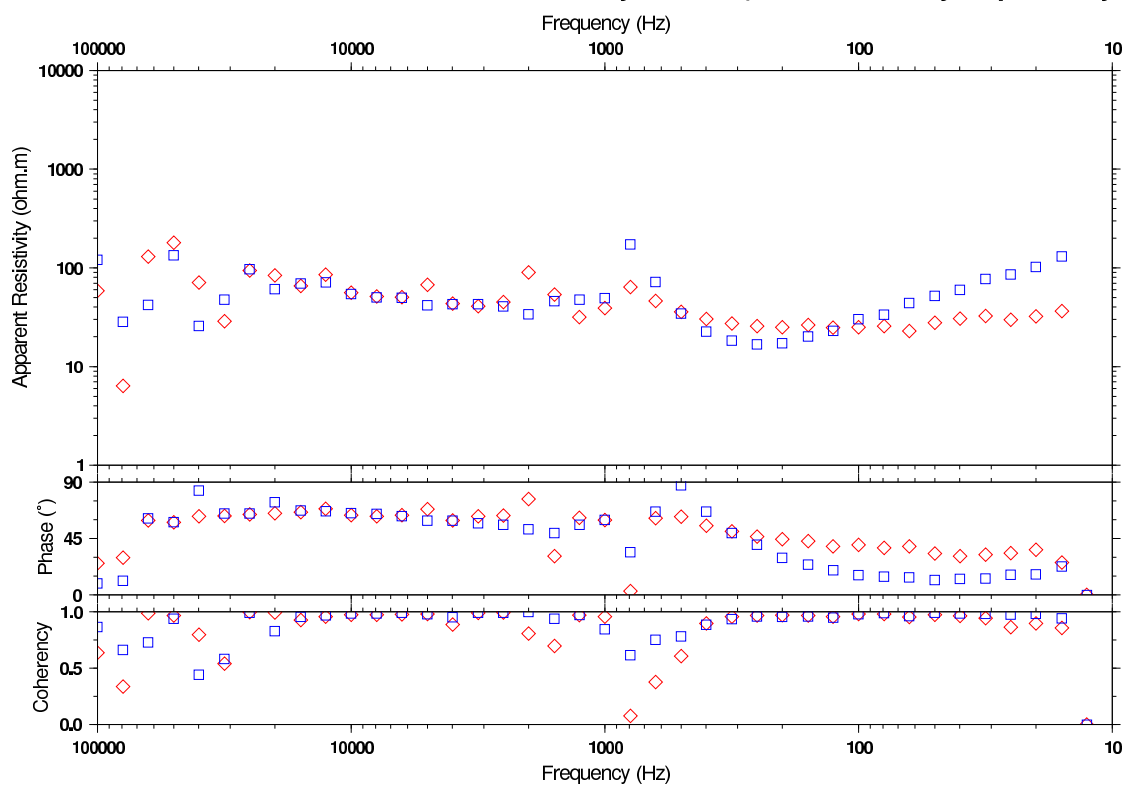

SVNA 12200m 088 - Scalar Res., Coherency, Phase (diamond=ExHy; square=EyHx)

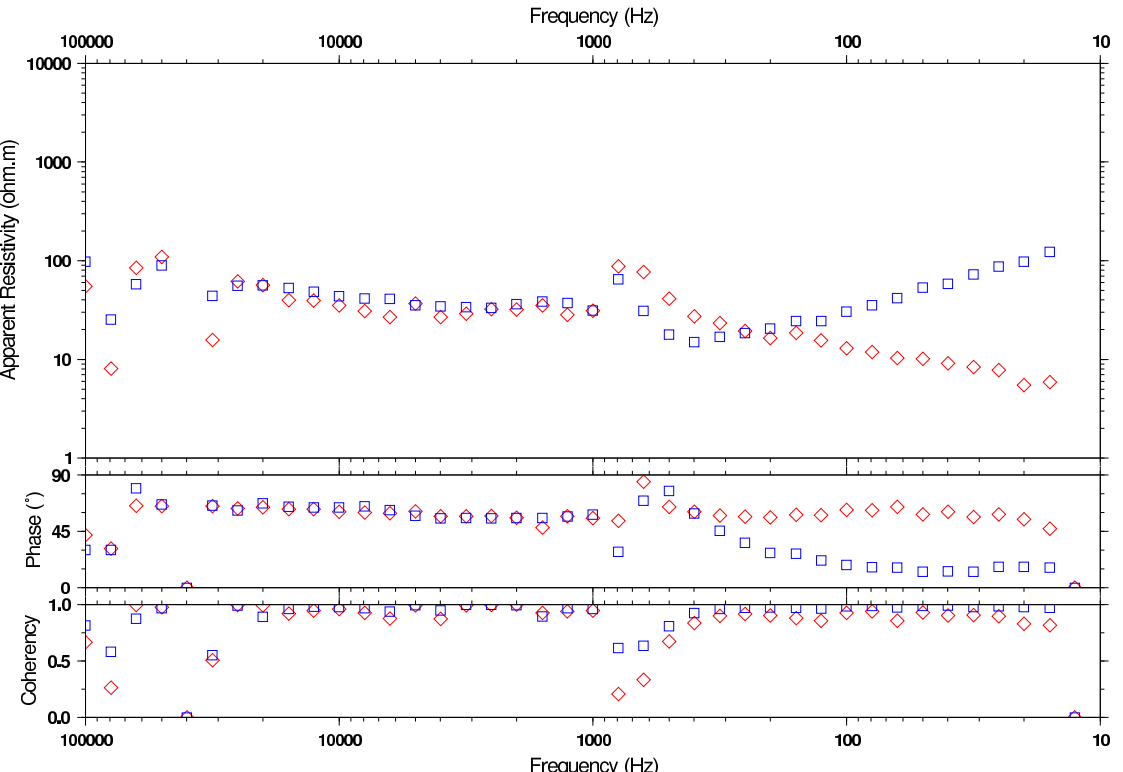

SVNA 12400m 093 - Scalar Res., Coherency, Phase (diamond=ExHy; square=EyHx)

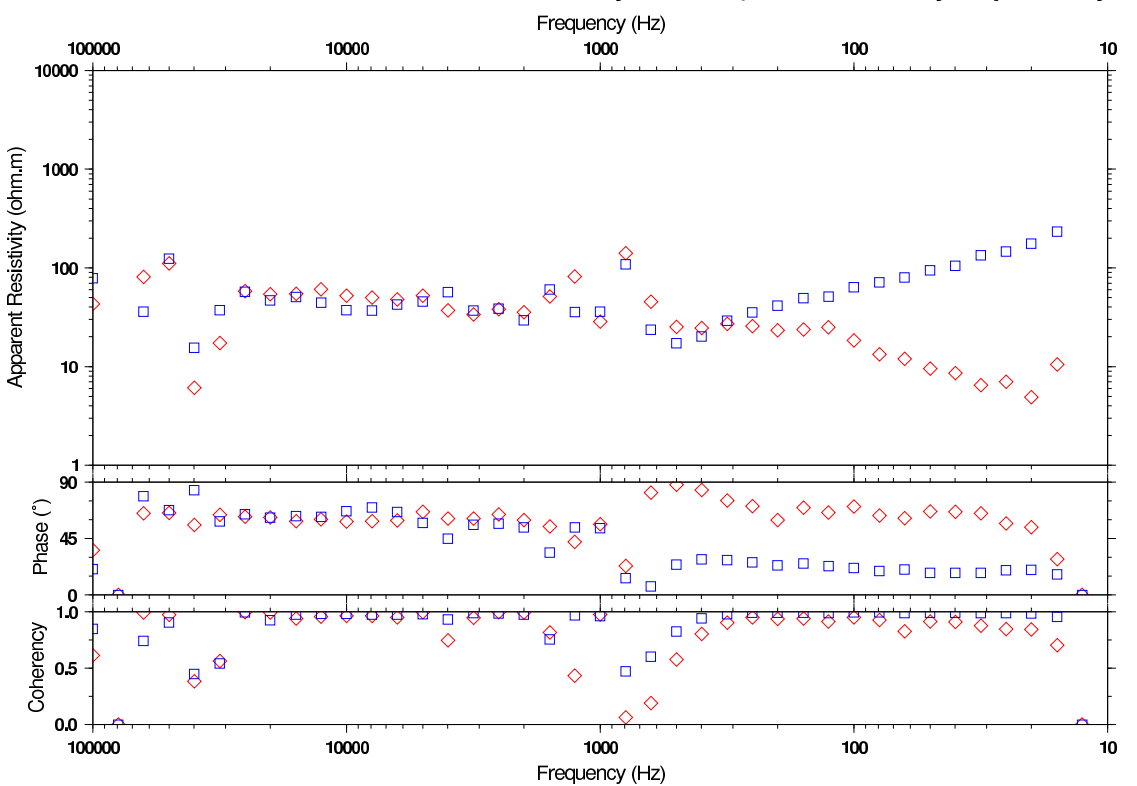

SVNA 12600m 102 - Scalar Res., Coherency, Phase (diamond=ExHy; square=EyHx)

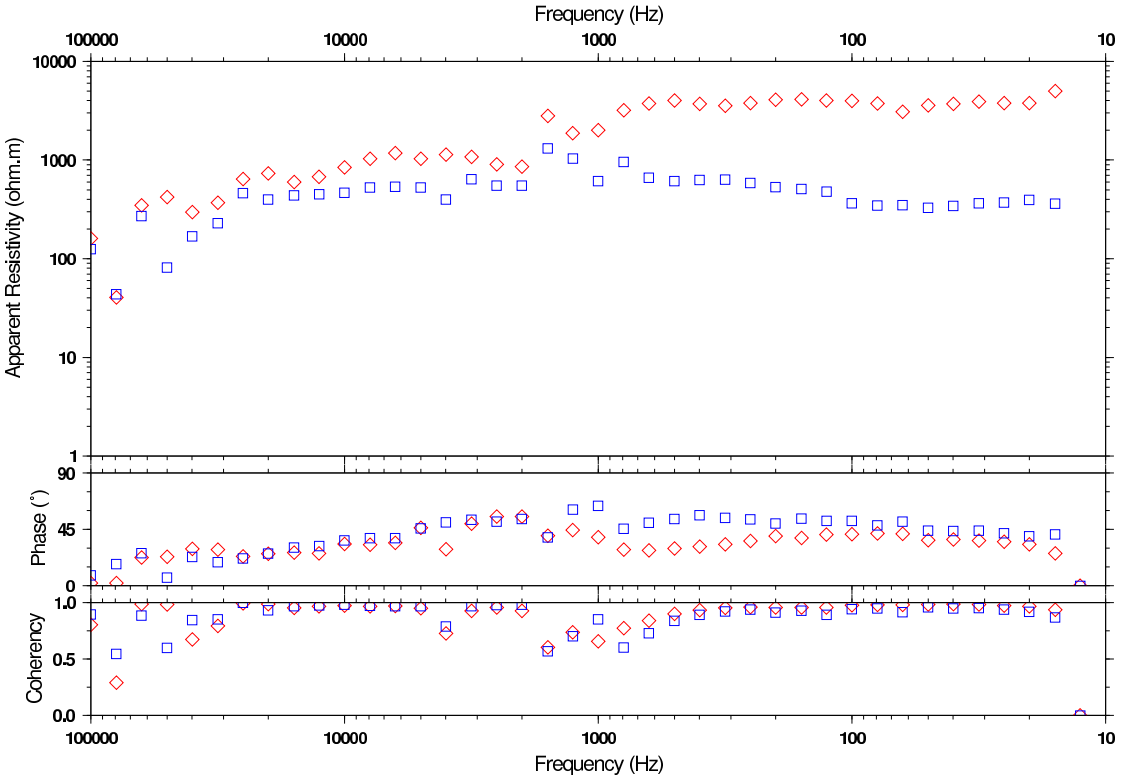


SVNB -0200m 107 - Scalar Res., Coherency, Phase (diamond=ExHy; square=EyHx)

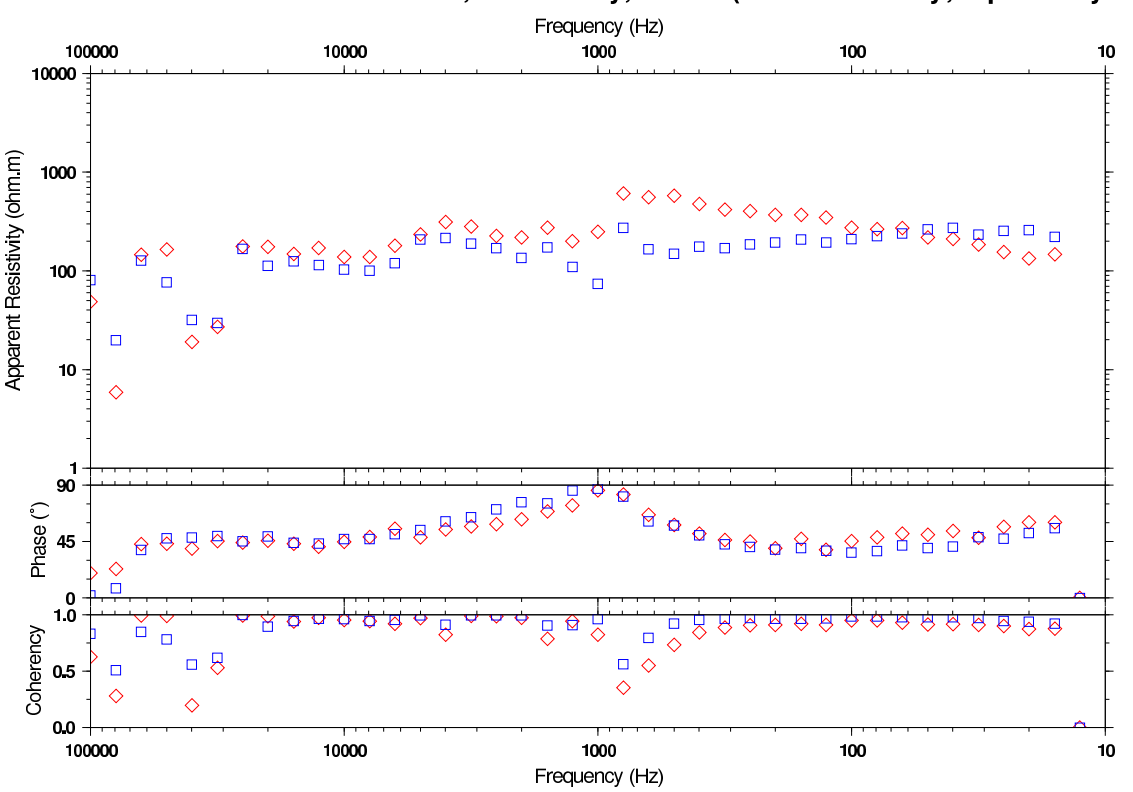

SVNB 00000m 103 - Scalar Res., Coherency, Phase (diamond=ExHy; square=EyHx)

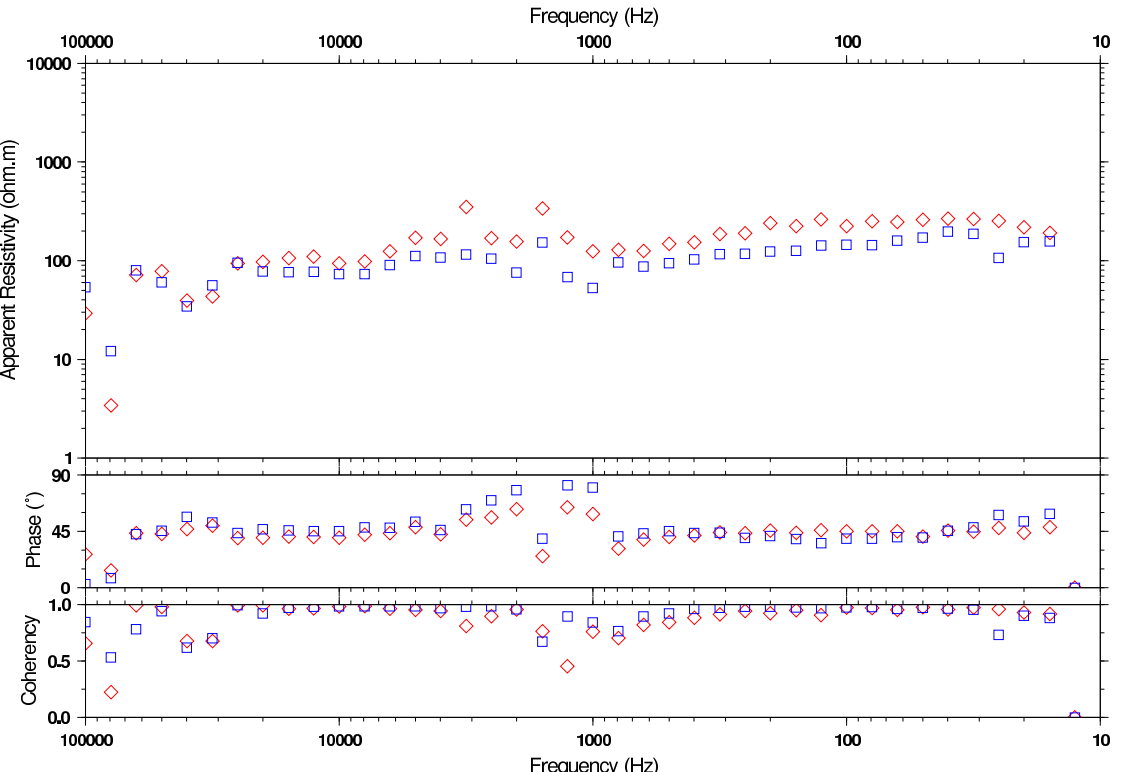

SVNB 00200m 108 - Scalar Res., Coherency, Phase (diamond=ExHy; square=EyHx)

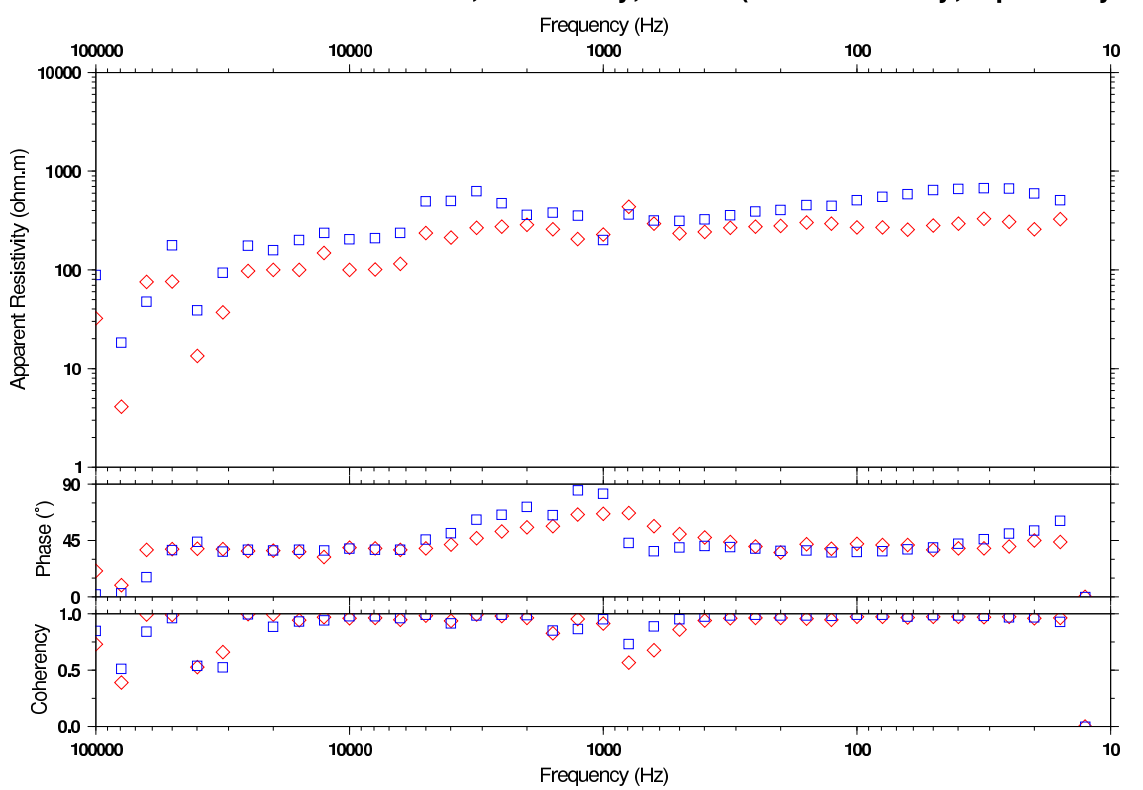

SVNB 00400m 104 - Scalar Res., Coherency, Phase (diamond=ExHy; square=EyHx)

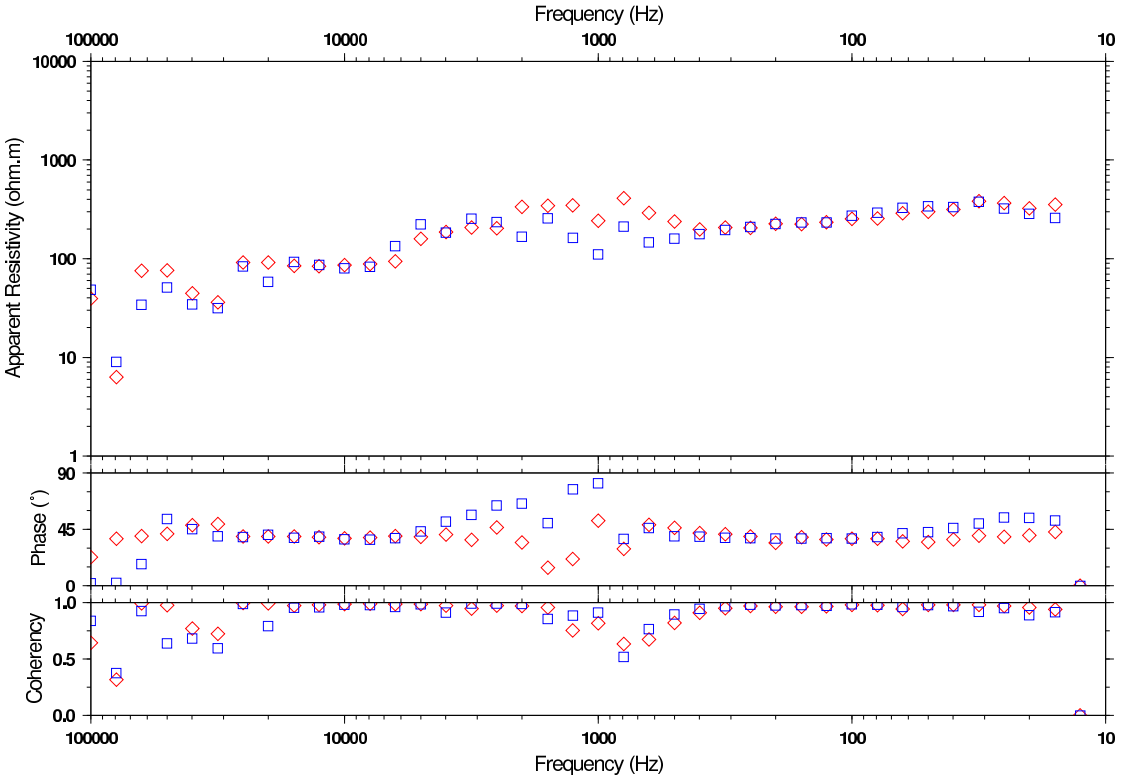




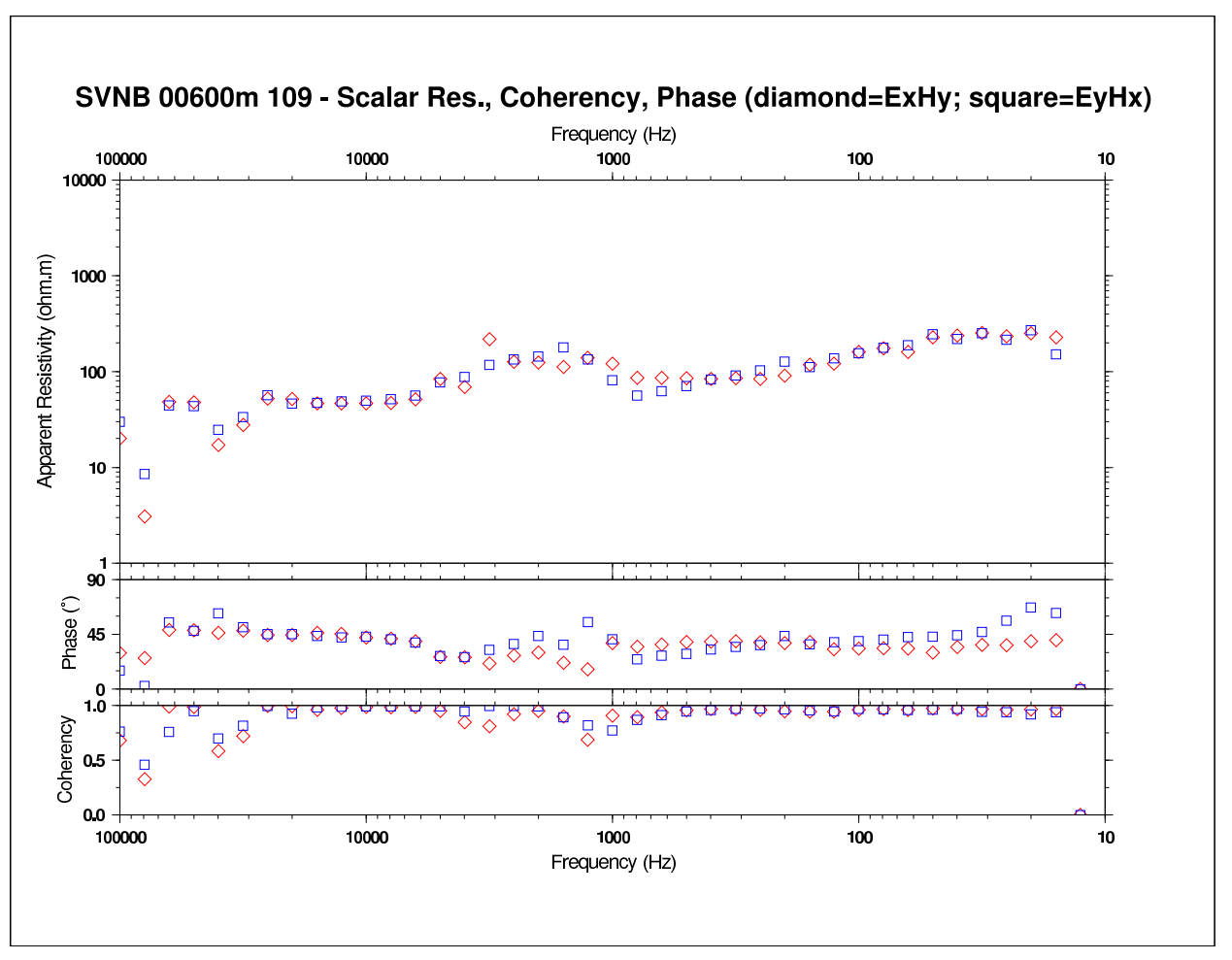

SVNB 01200m 106 - Scalar Res., Coherency, Phase (diamond=ExHy; square=EyHx)

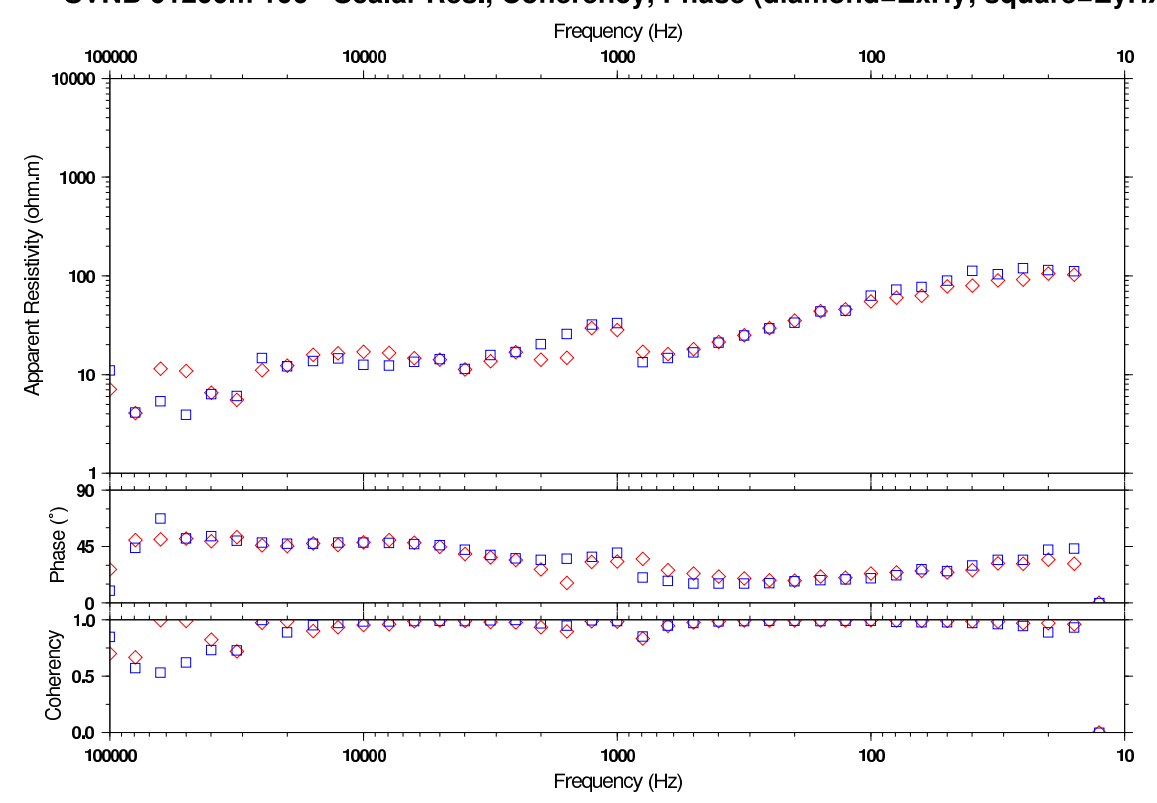

SVNB 00800m 105 - Scalar Res., Coherency, Phase (diamond=ExHy; square=EyHx)

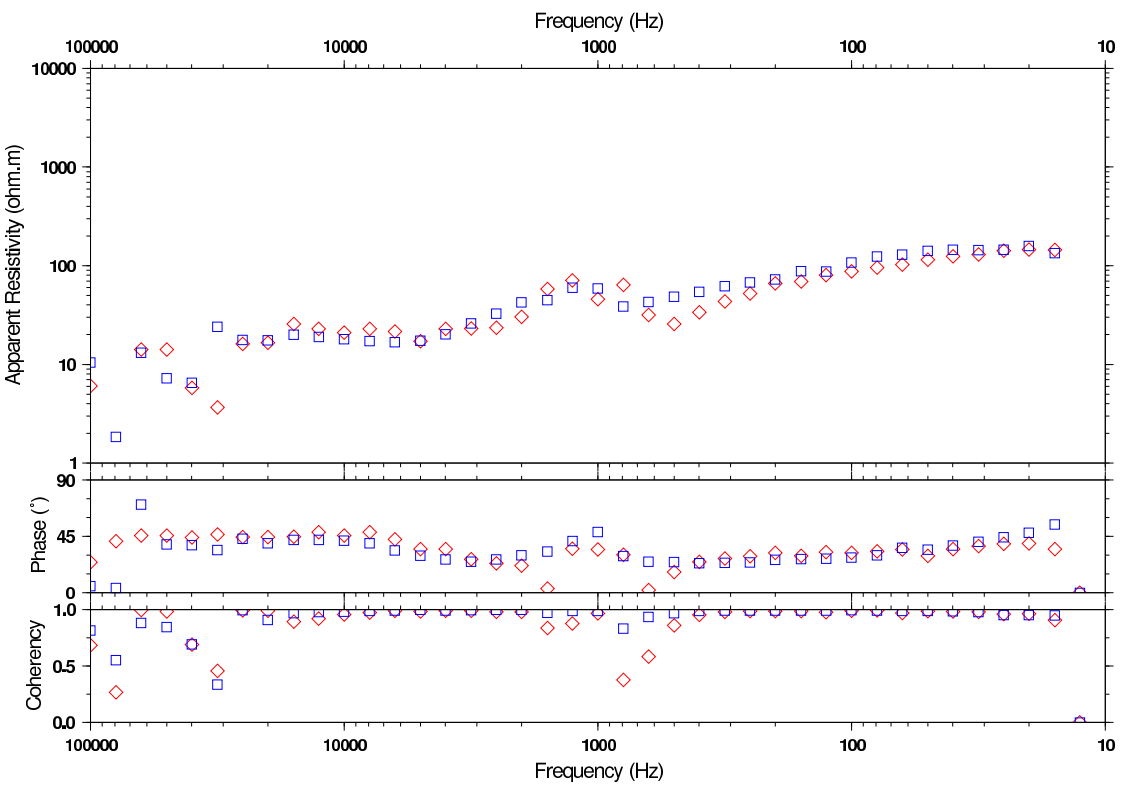

SVNB 01600m 111 - Scalar Res., Coherency, Phase (diamond=ExHy; square=EyHx)

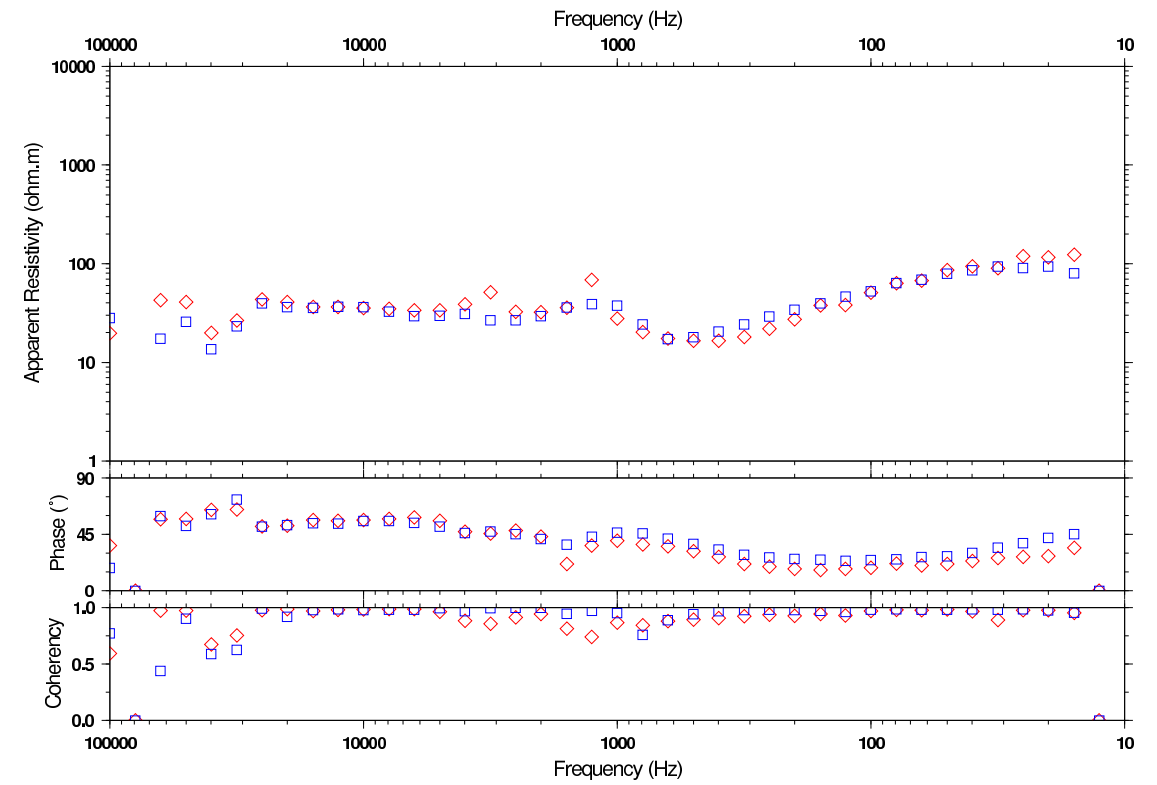


SVNB 02000m 112 - Scalar Res., Coherency, Phase (diamond=ExHy; square=EyHx)

100000 $\underset{1000}{\text { Frequency }(\mathrm{Hz})}$

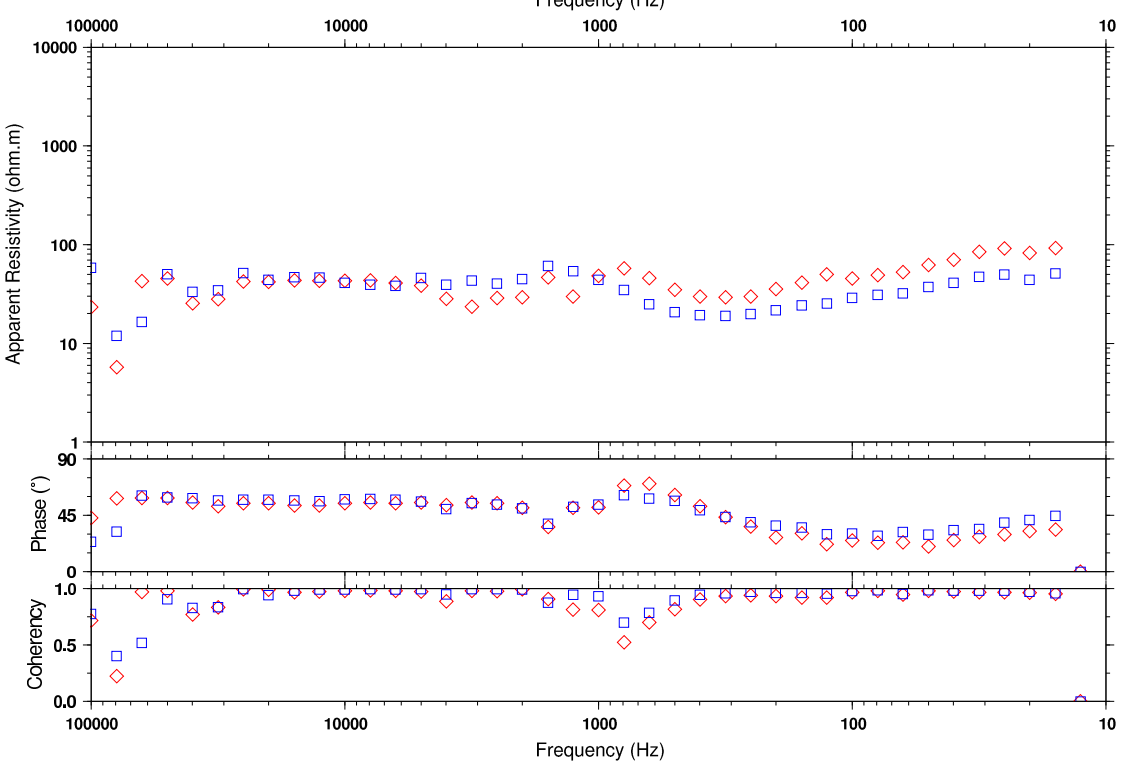


SVNC -0600m 016 - Scalar Res., Coherency, Phase (diamond=ExHy; square=EyHx)

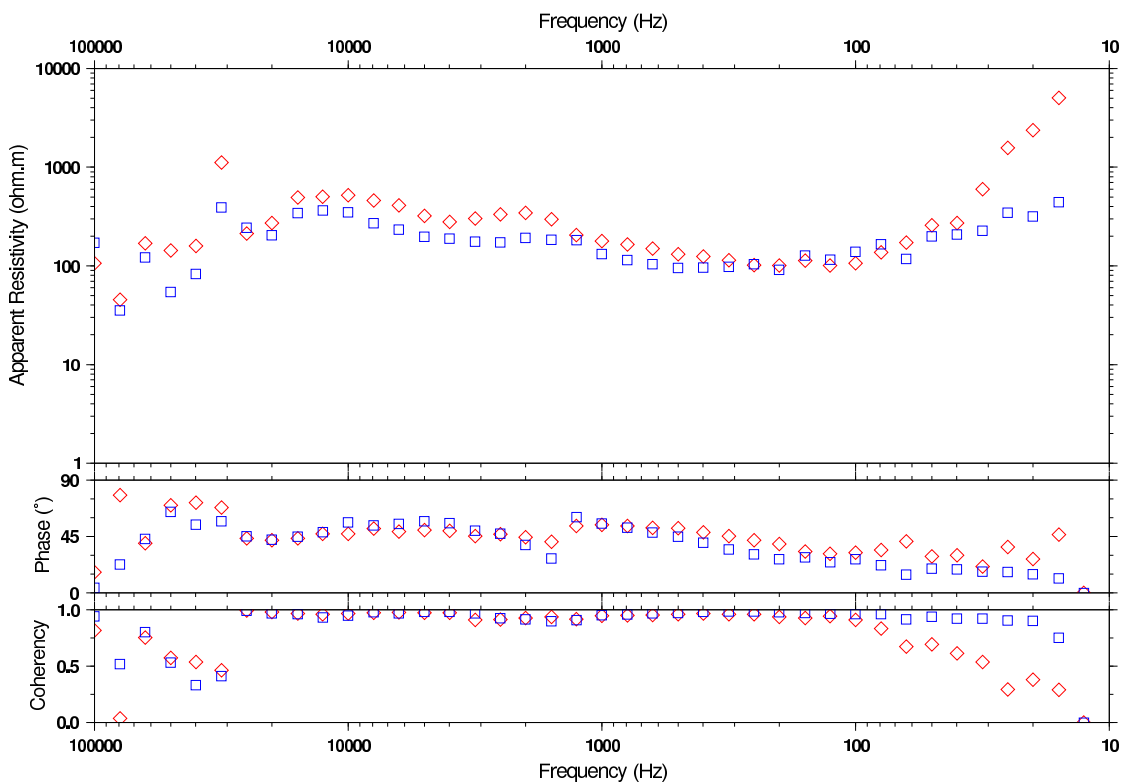

SVNC -0400m 027 - Scalar Res., Coherency, Phase (diamond=ExHy; square=EyHx)

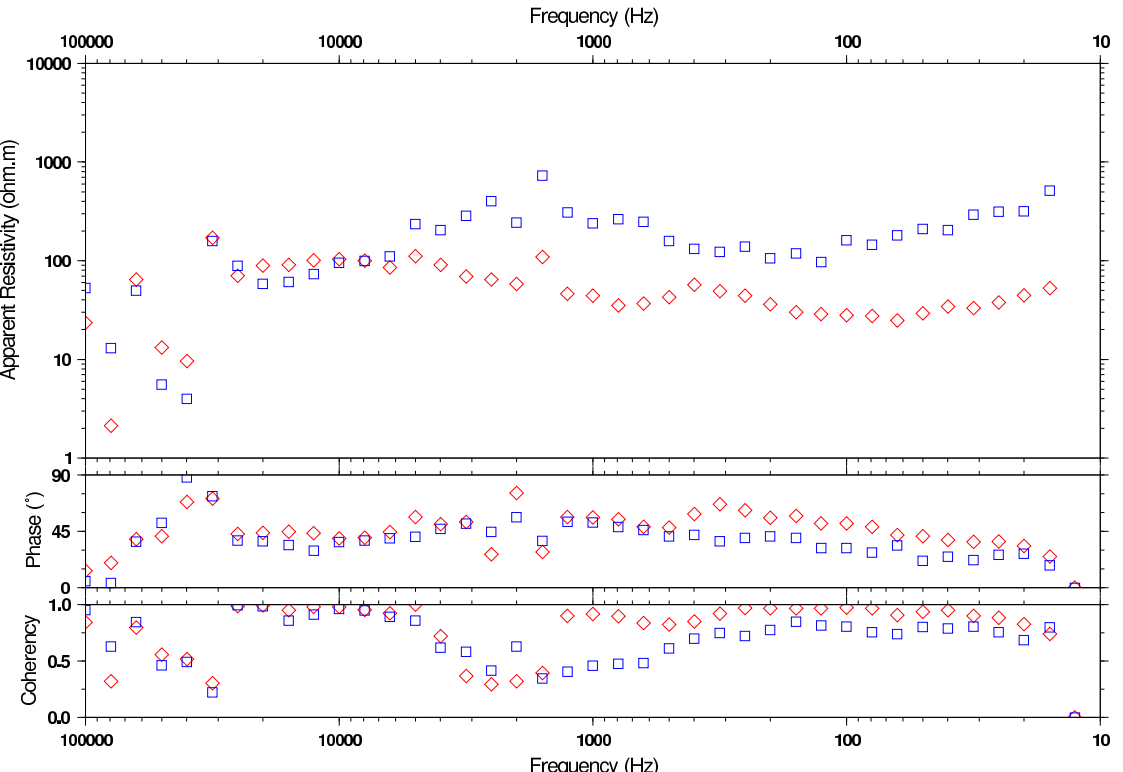

SVNC -0200m 019 - Scalar Res., Coherency, Phase (diamond=ExHy; square=EyHx)

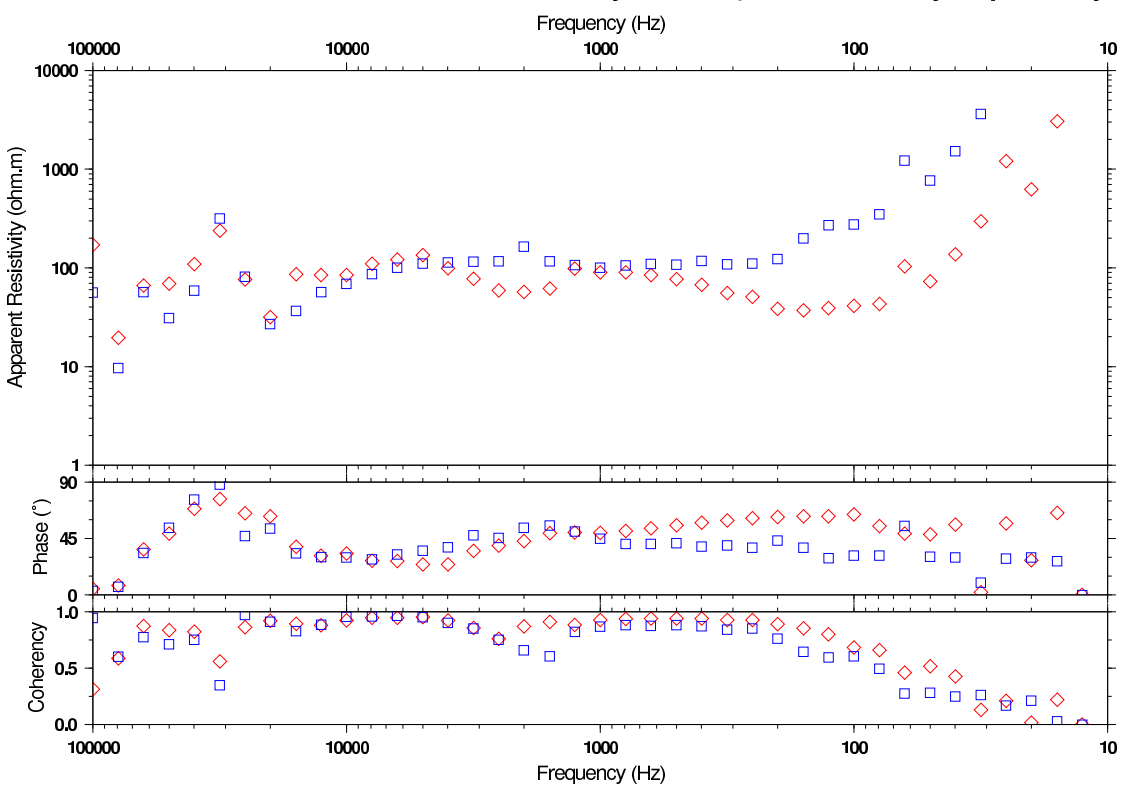

SVNC 00000m 021 - Scalar Res., Coherency, Phase (diamond=ExHy; square=EyHx)

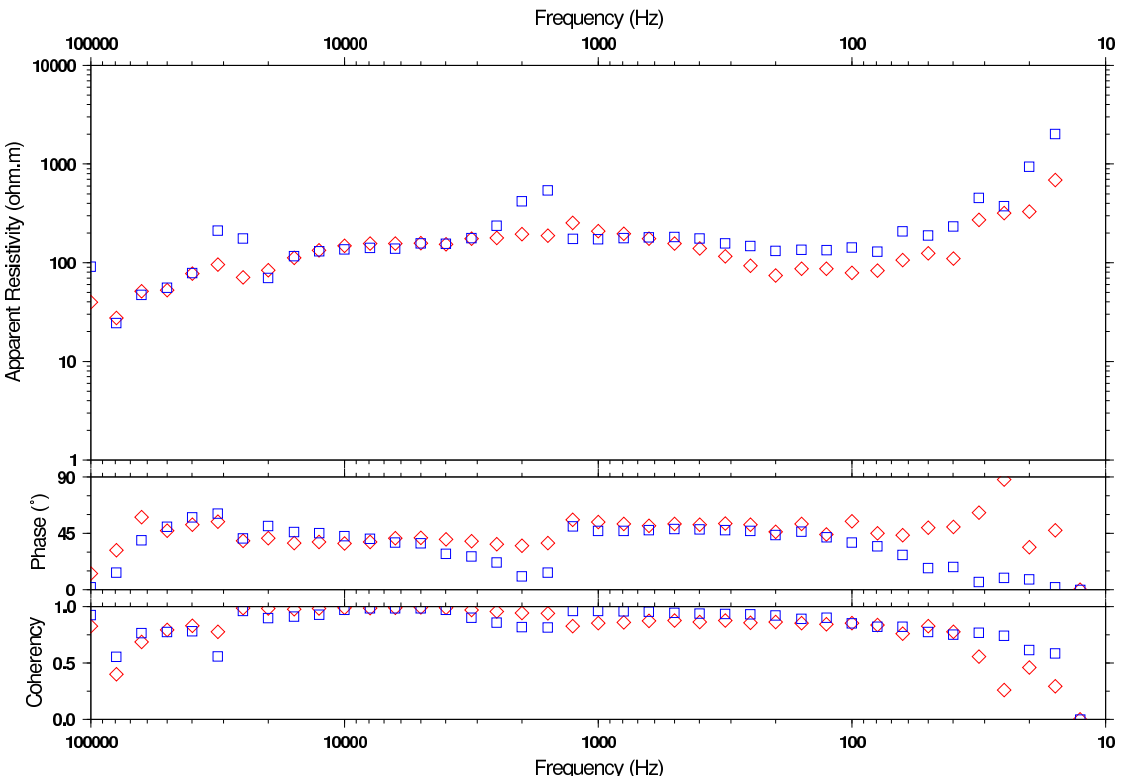




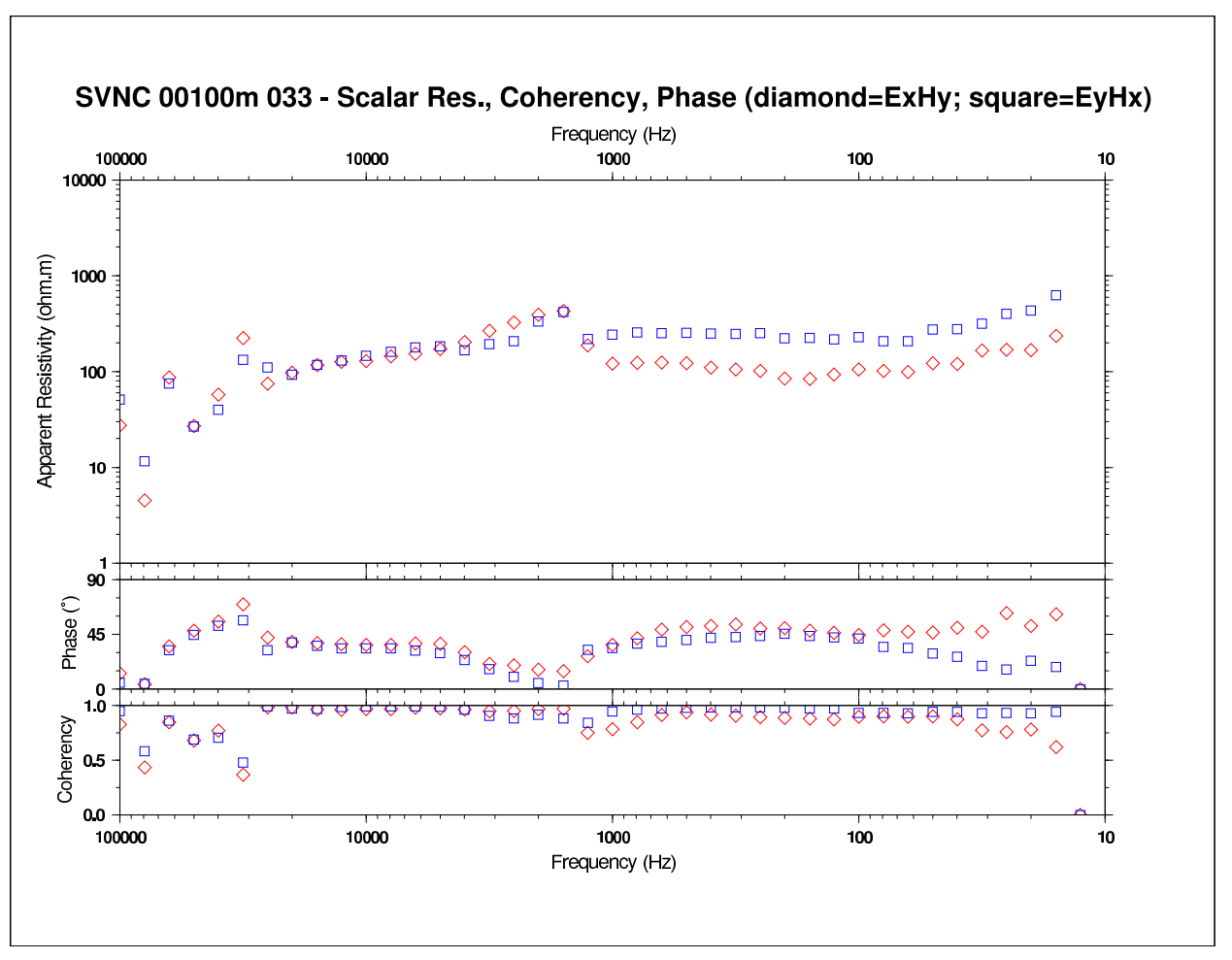

SVNC 00400m 023 - Scalar Res., Coherency, Phase (diamond=ExHy; square=EyHx)

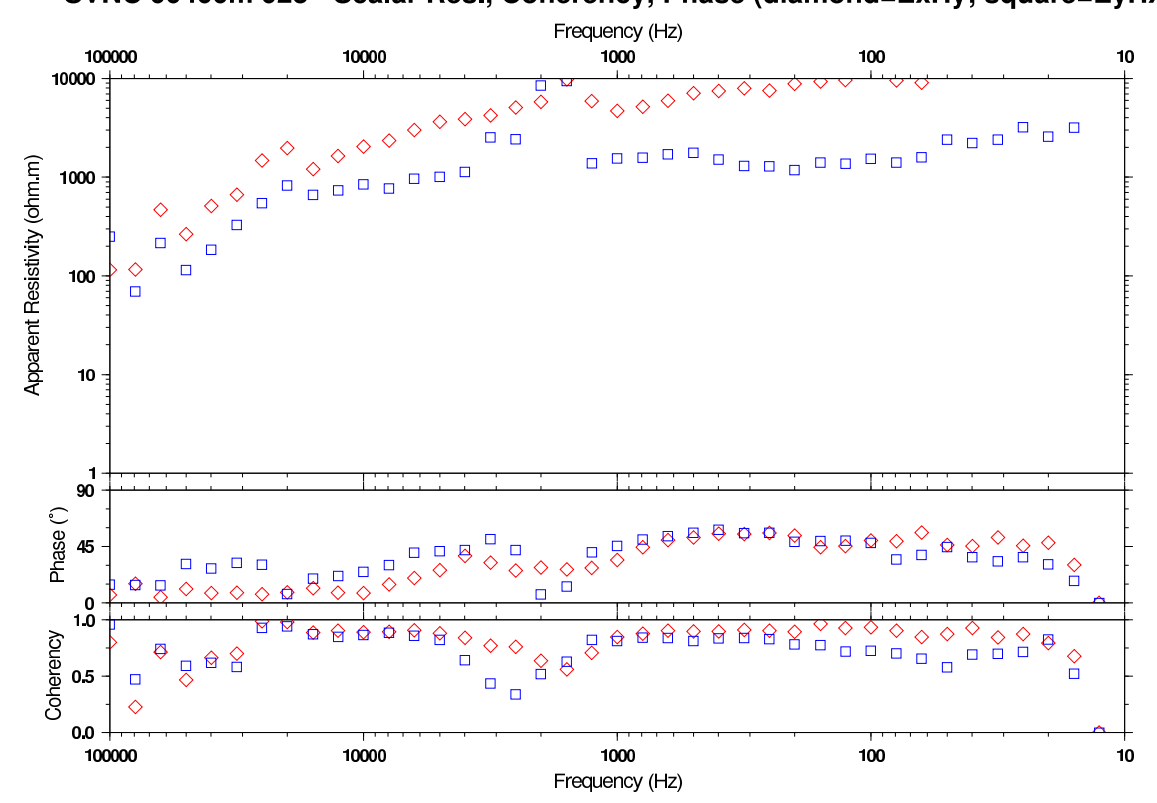

SVNC 00200m 032 - Scalar Res., Coherency, Phase (diamond=ExHy; square=EyHx)

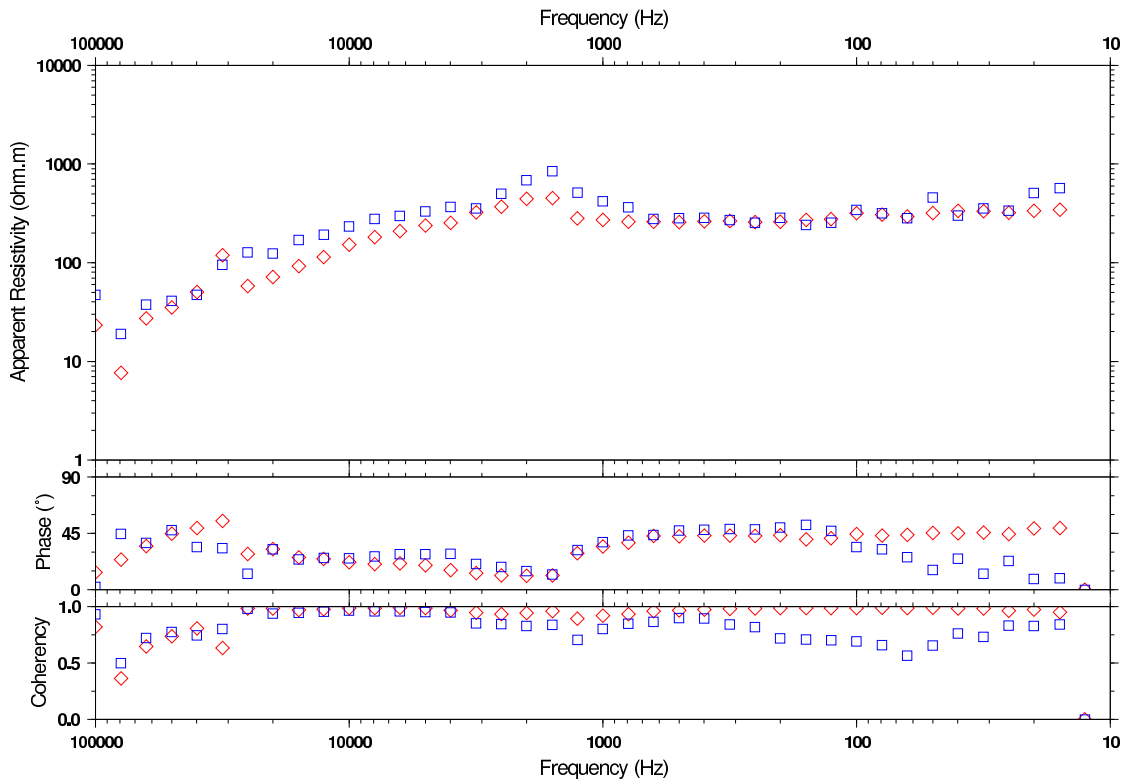

SVNC 00600m 025 - Scalar Res., Coherency, Phase (diamond=ExHy; square=EyHx)

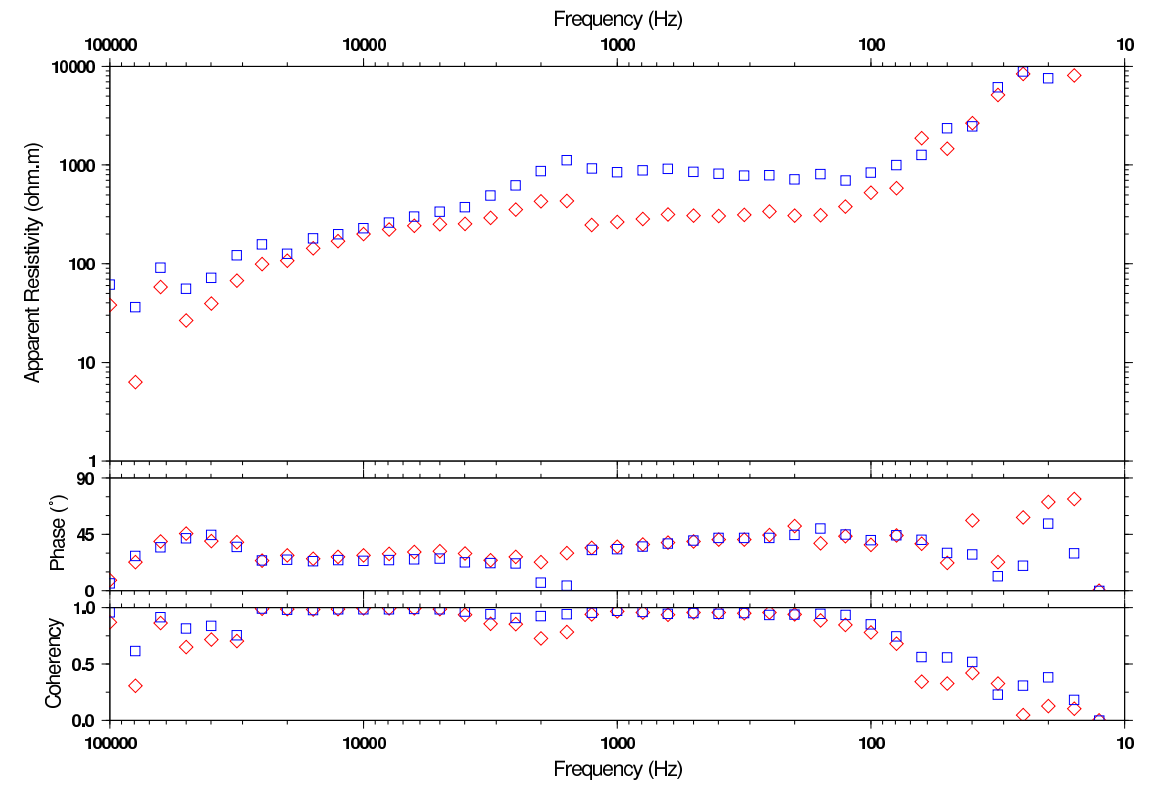


SVNC 00800m 030 - Scalar Res., Coherency, Phase (diamond=ExHy; square=EyHx)

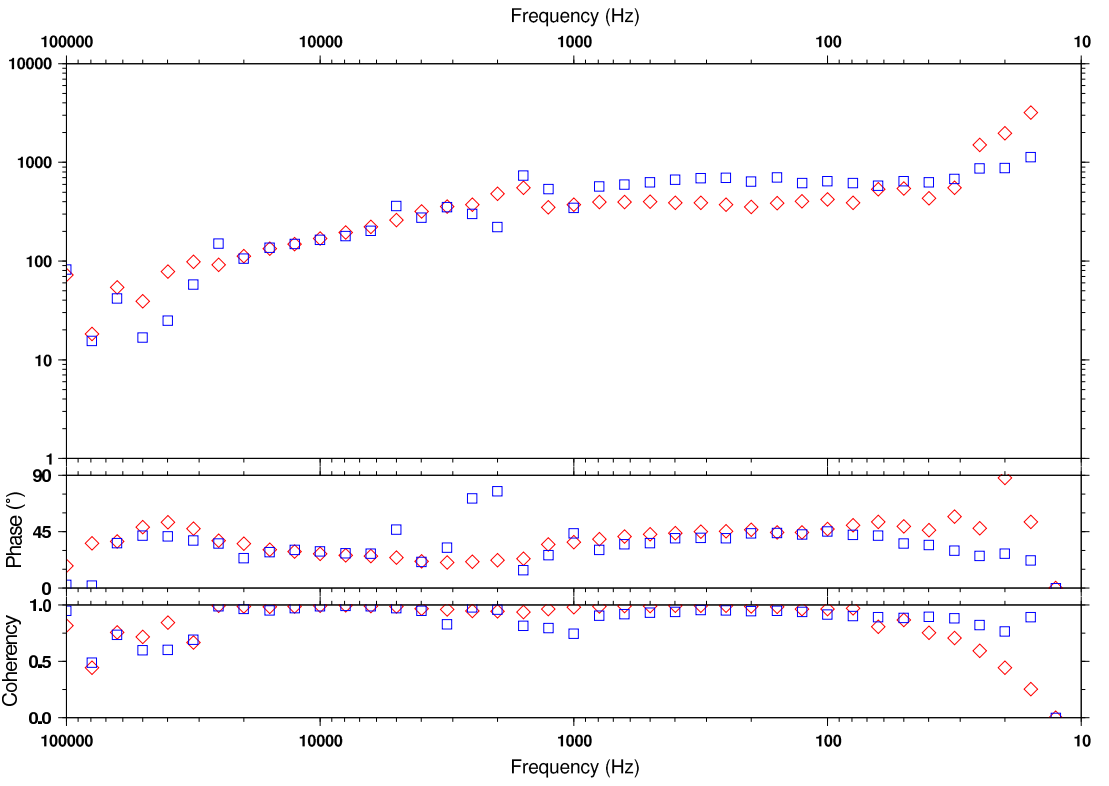


SVNE -1800m 016 - Scalar Res., Coherency, Phase (diamond=ExHy; square=EyHx)

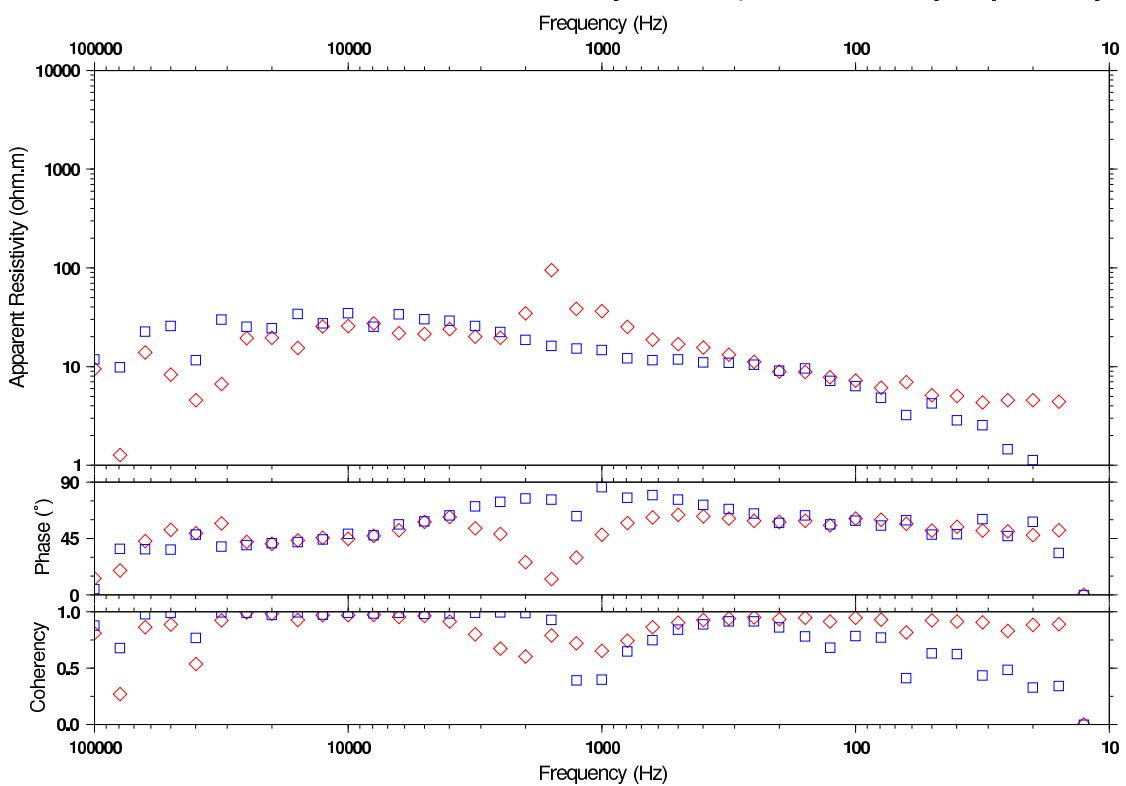

SVNE -1400m 014 - Scalar Res., Coherency, Phase (diamond=ExHy; square=EyHx)

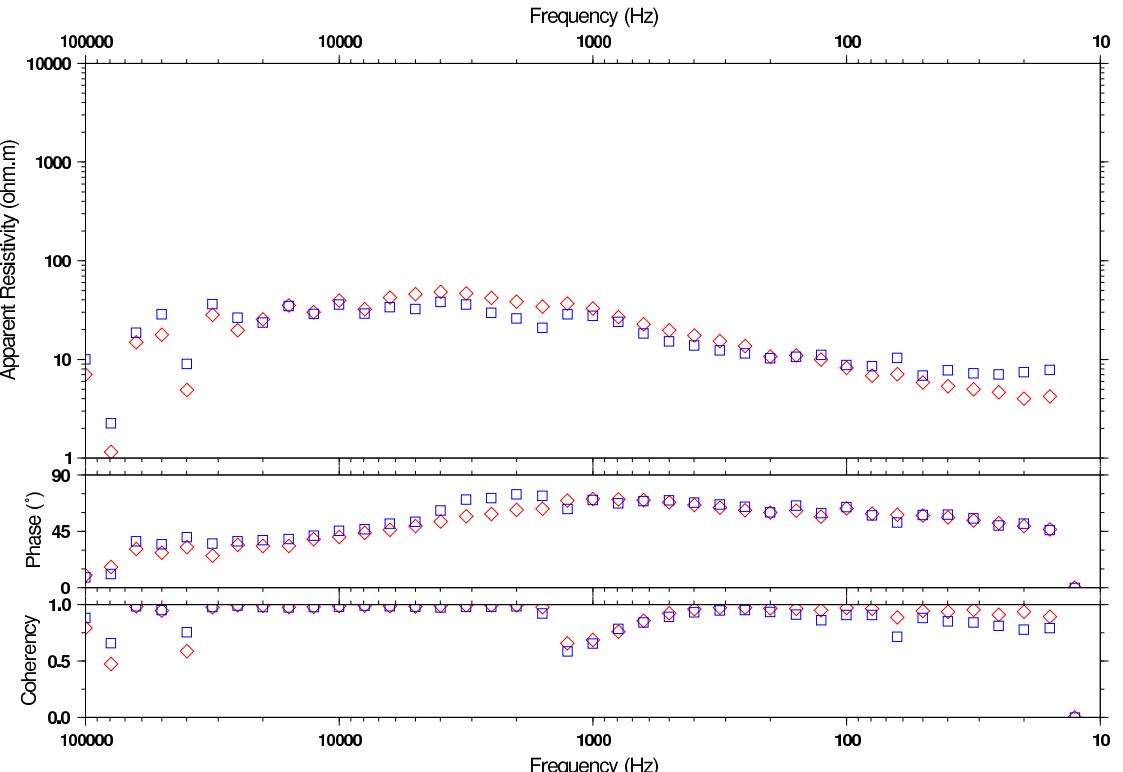

SVNE -1000m 013 - Scalar Res., Coherency, Phase (diamond=ExHy; square=EyHx)

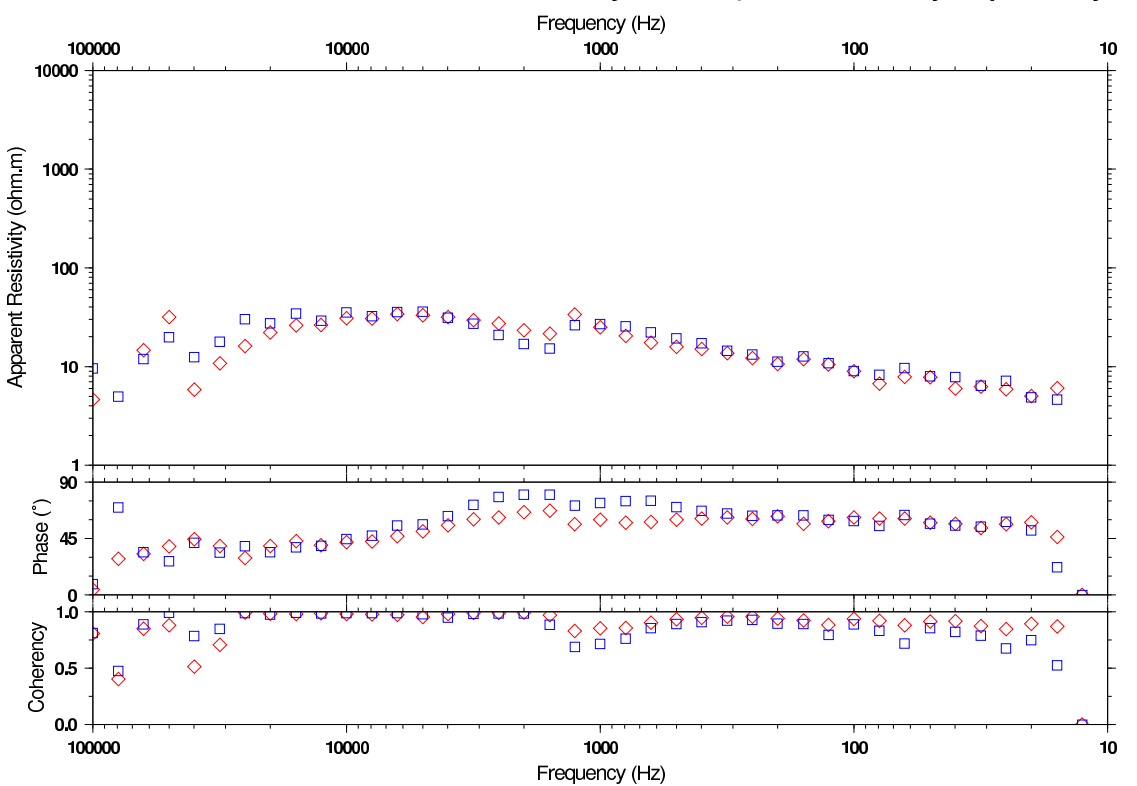

SVNE -0800m 012 - Scalar Res., Coherency, Phase (diamond=ExHy; square=EyHx)

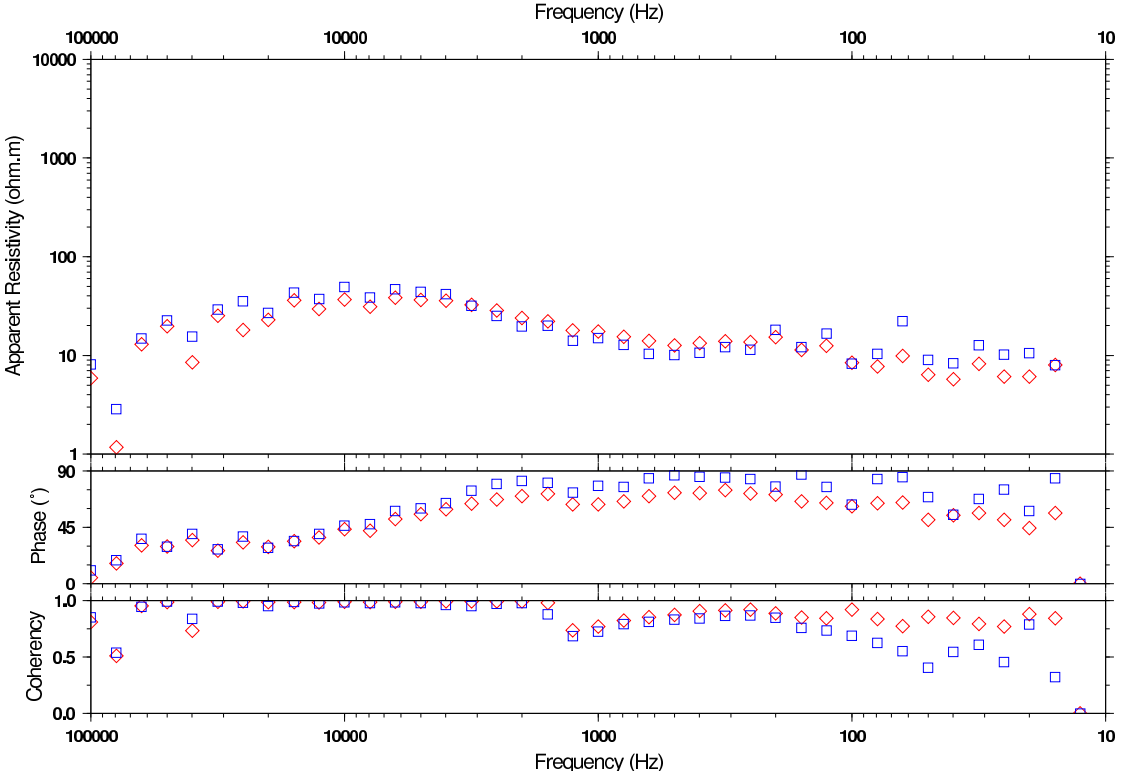


SVNE -0600m 010 - Scalar Res., Coherency, Phase (diamond=ExHy; square=EyHx)

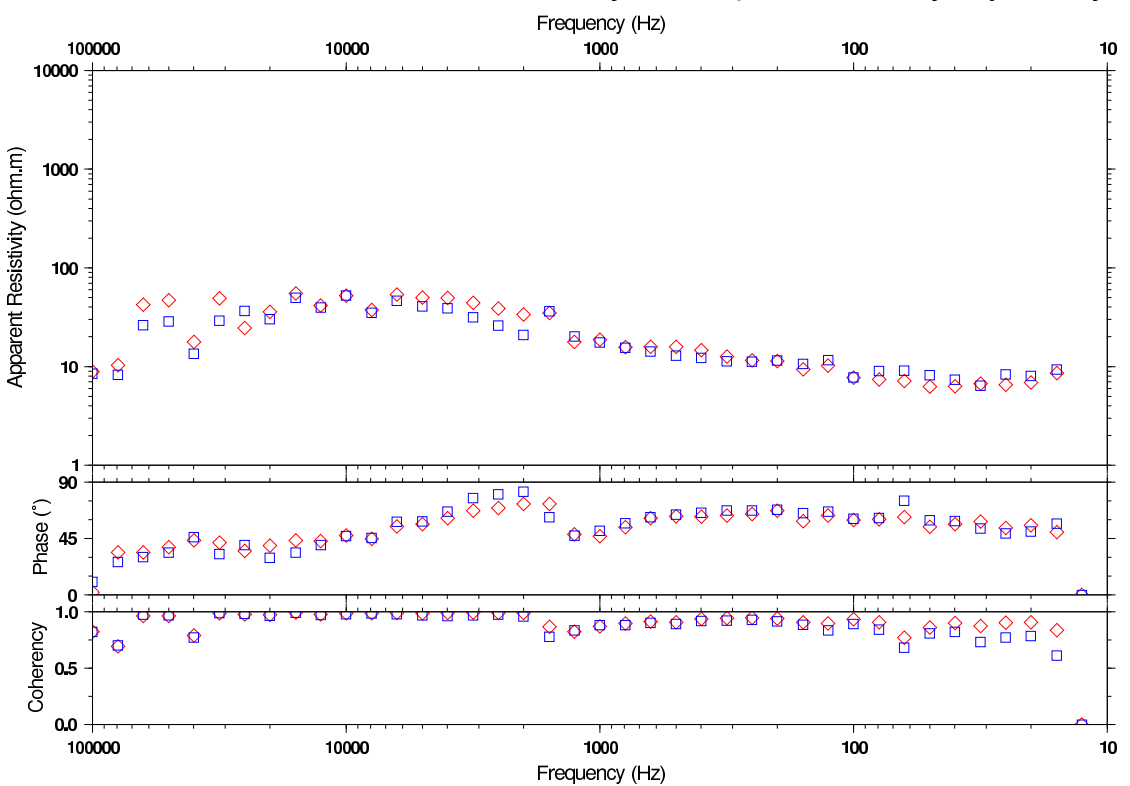

SVNE -0400m 007 - Scalar Res., Coherency, Phase (diamond=ExHy; square=EyHx)

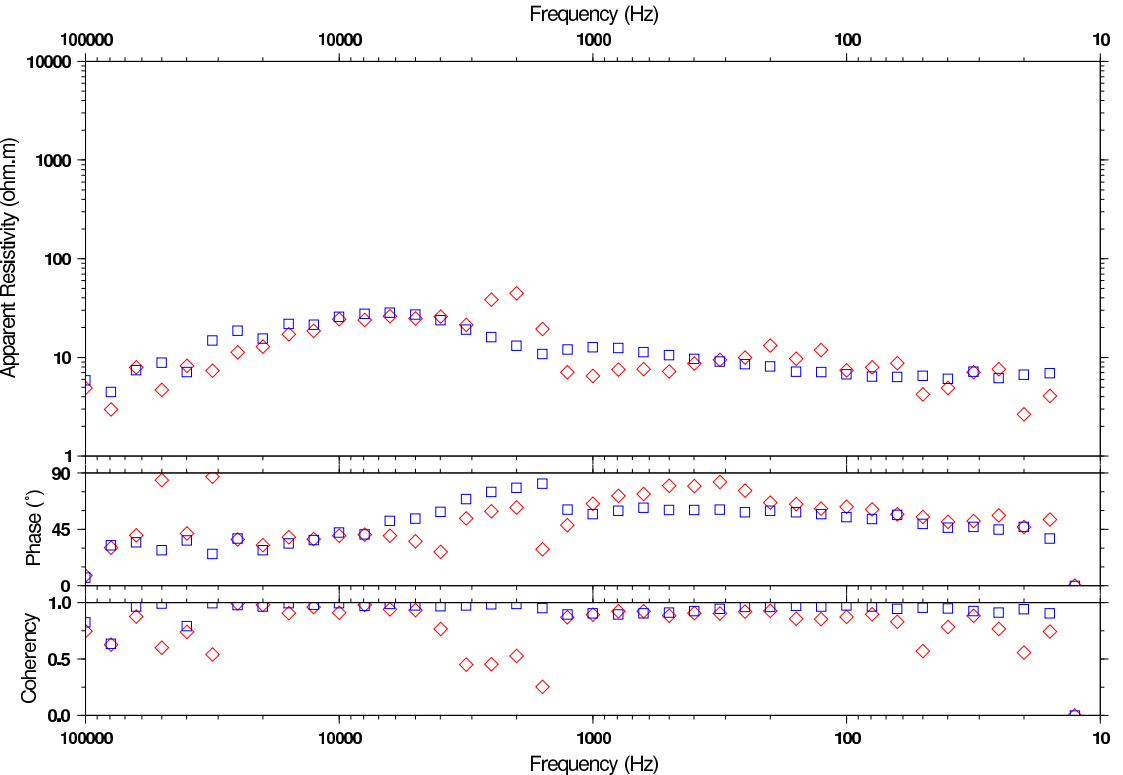

SVNE -0200m 041 - Scalar Res., Coherency, Phase (diamond=ExHy; square=EyHx)

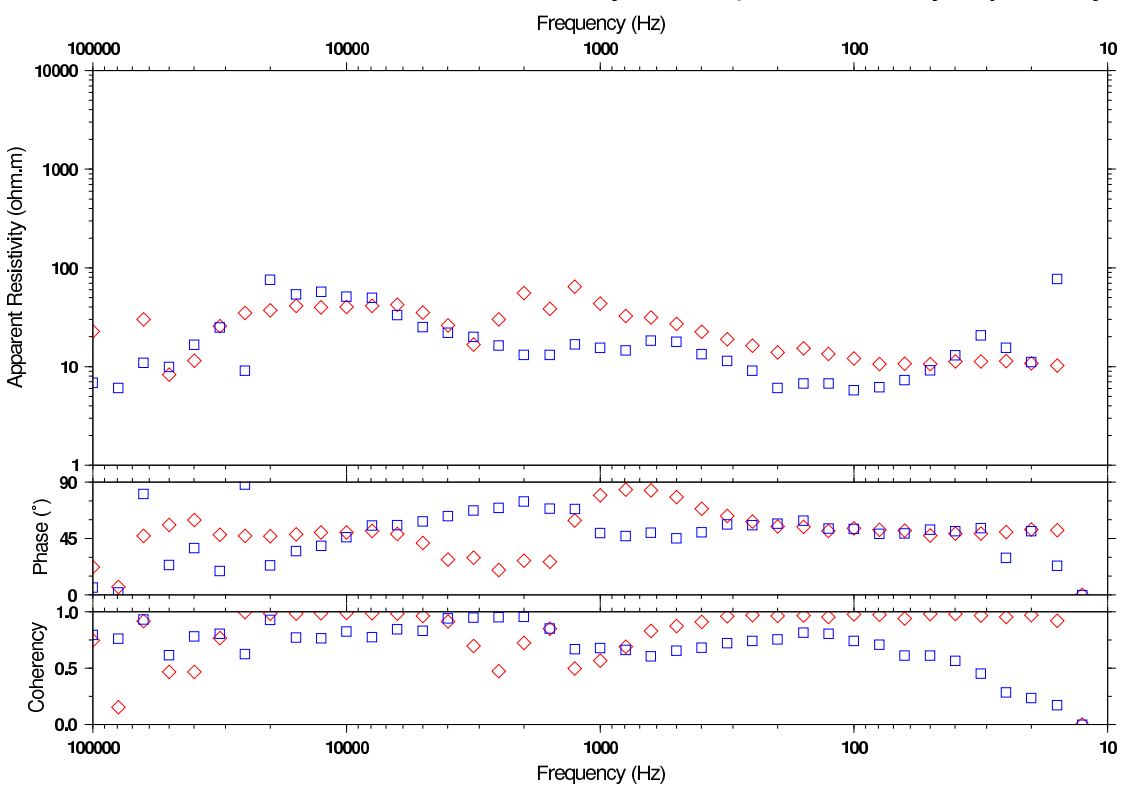

SVNE 00000m 004 - Scalar Res., Coherency, Phase (diamond=ExHy; square=EyHx)

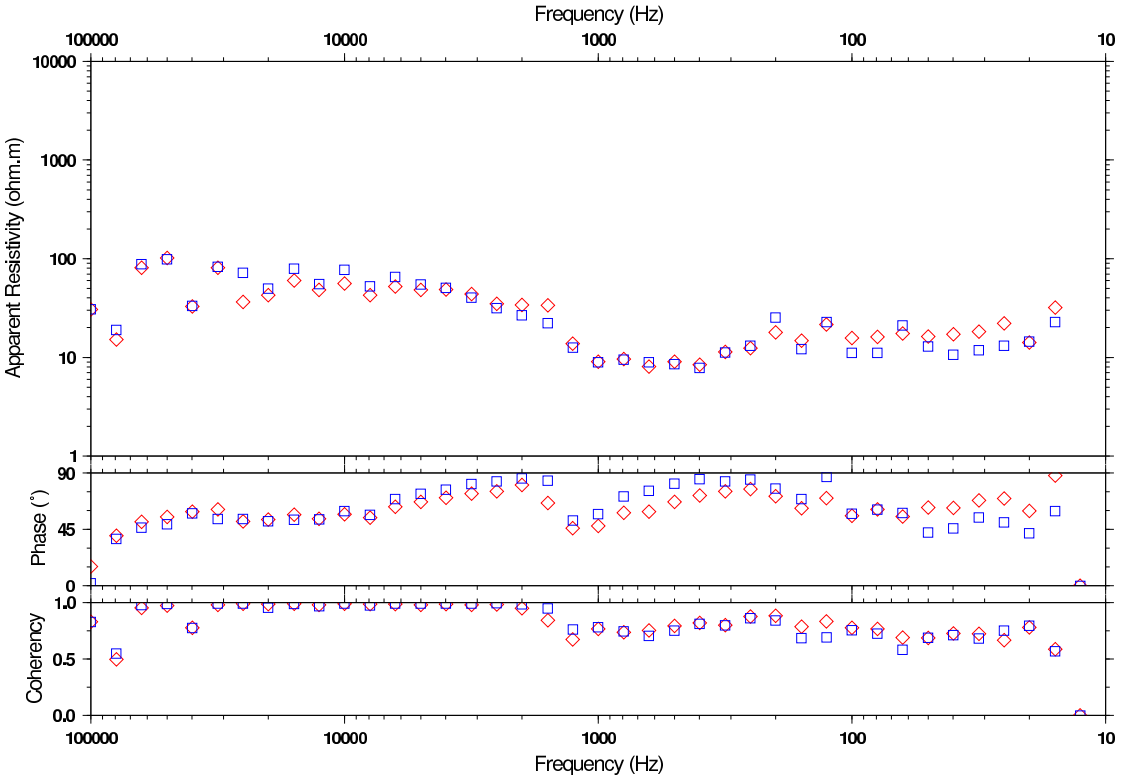




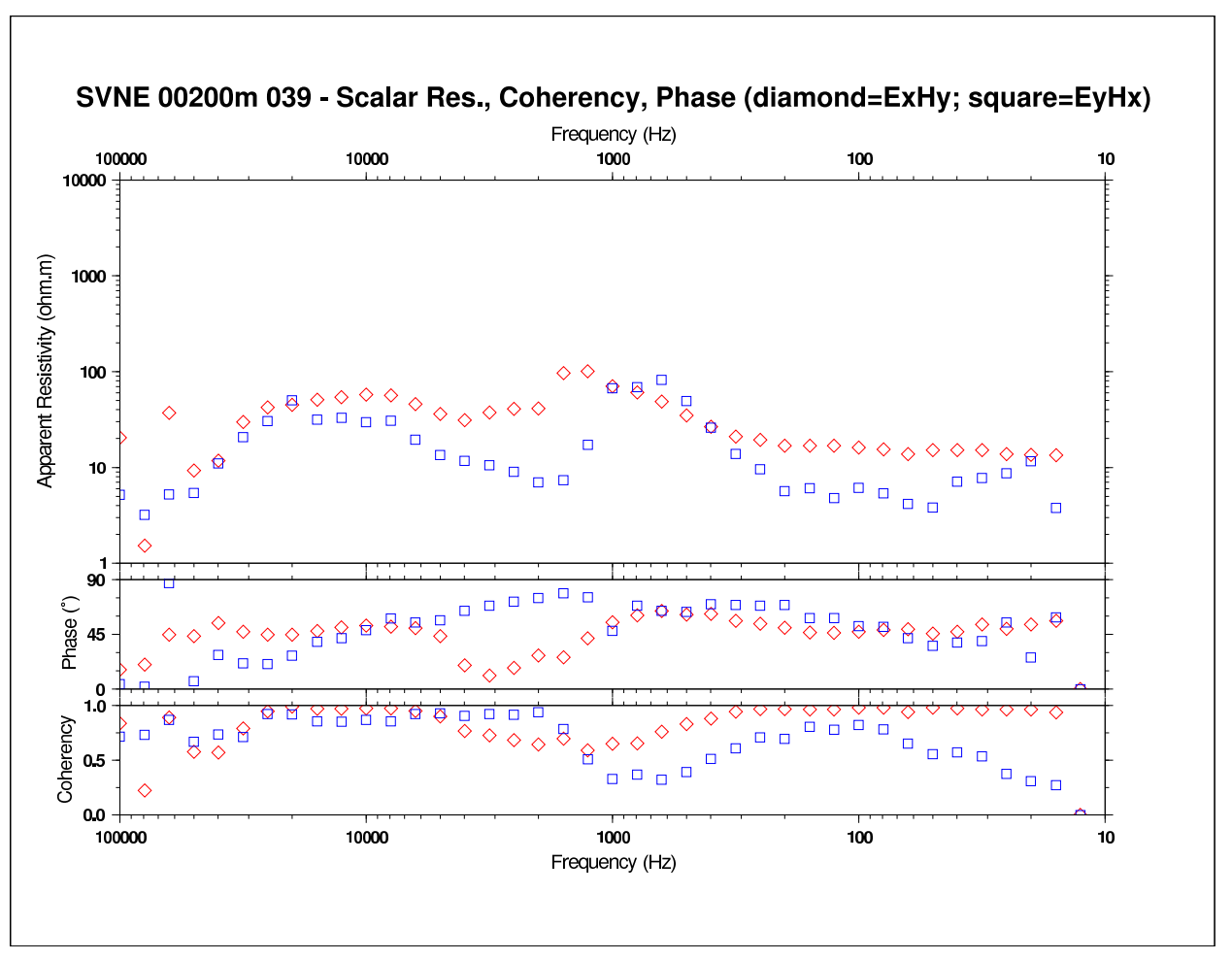

SVNE 00600m 023 - Scalar Res., Coherency, Phase (diamond=ExHy; square=EyHx)

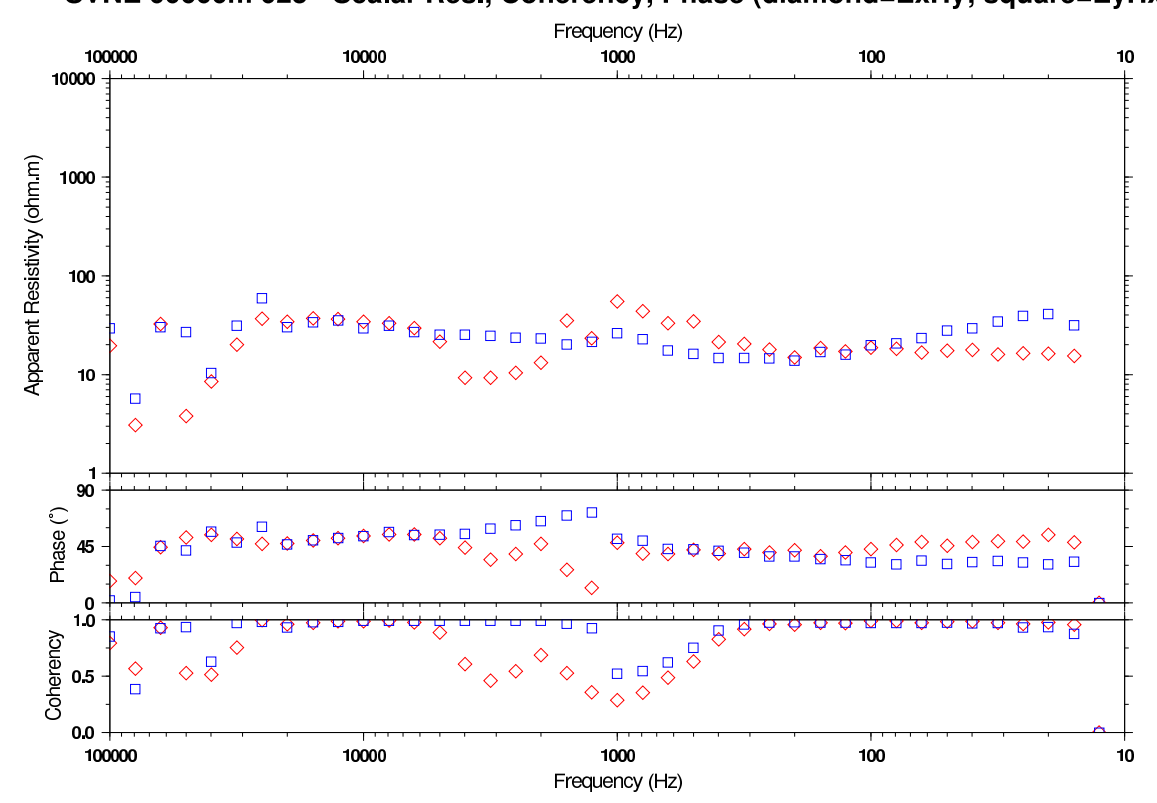

SVNE 00400m 019 - Scalar Res., Coherency, Phase (diamond=ExHy; square=EyHx)

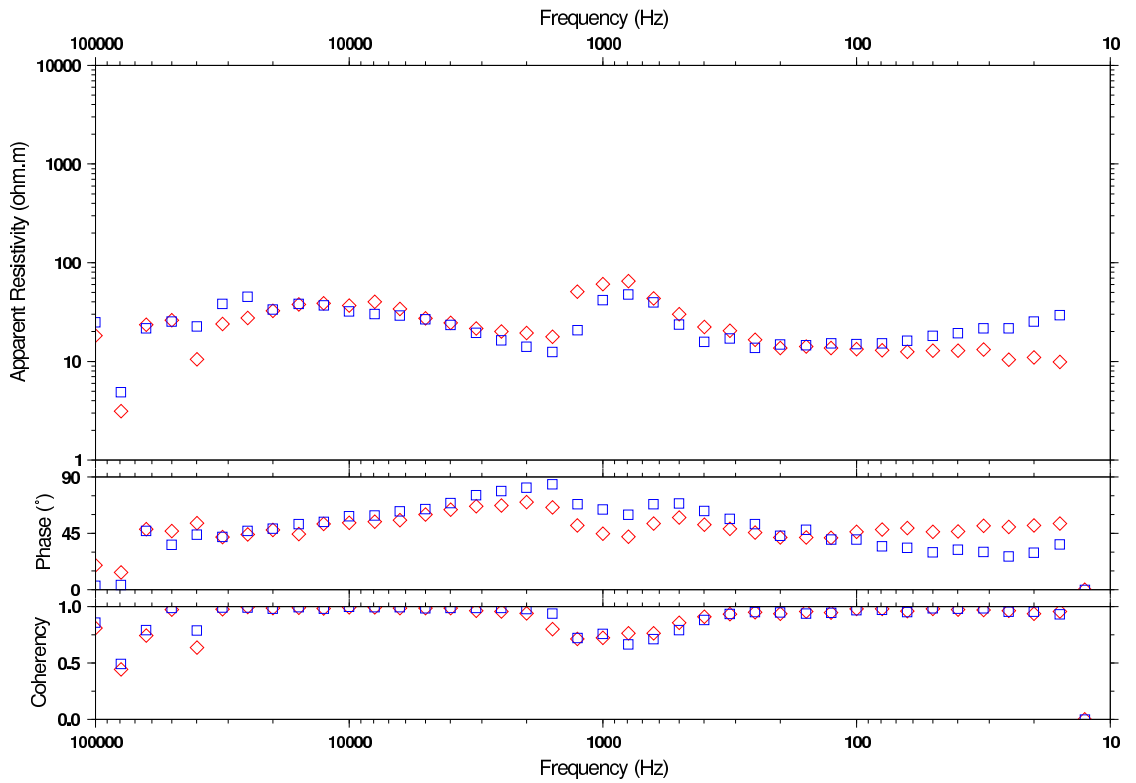

SVNE 00800m 036 - Scalar Res., Coherency, Phase (diamond=ExHy; square=EyHx)

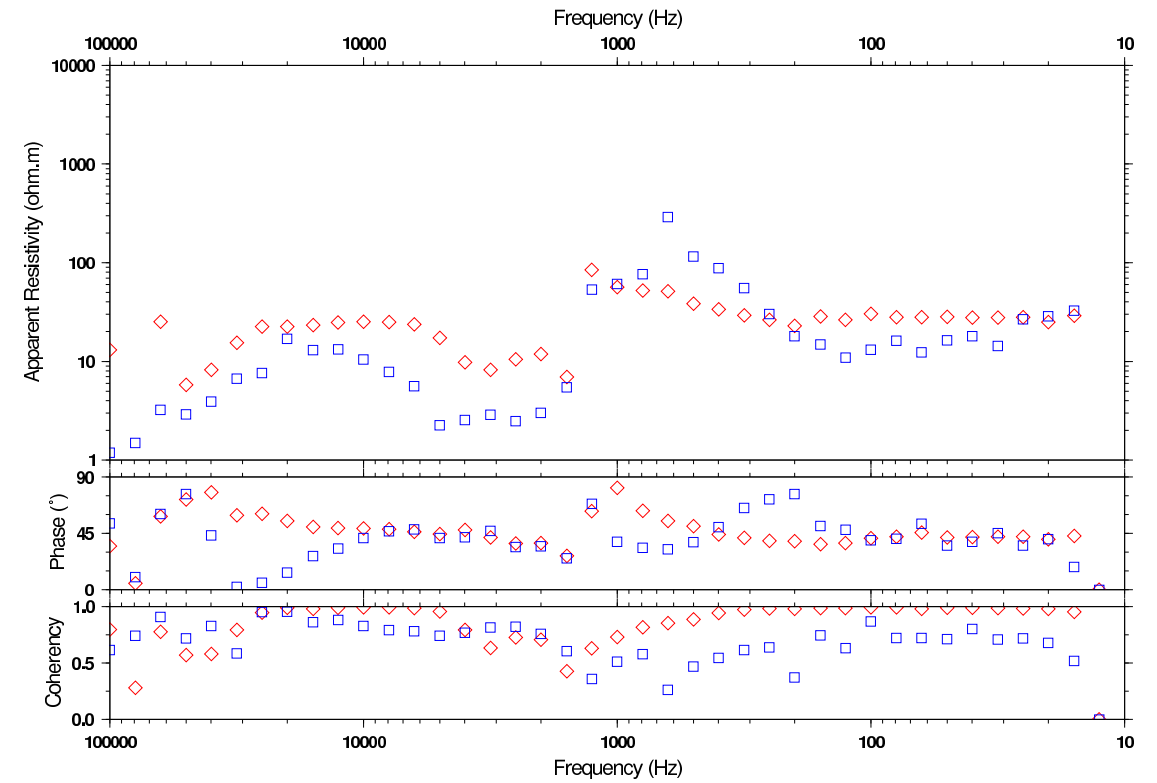



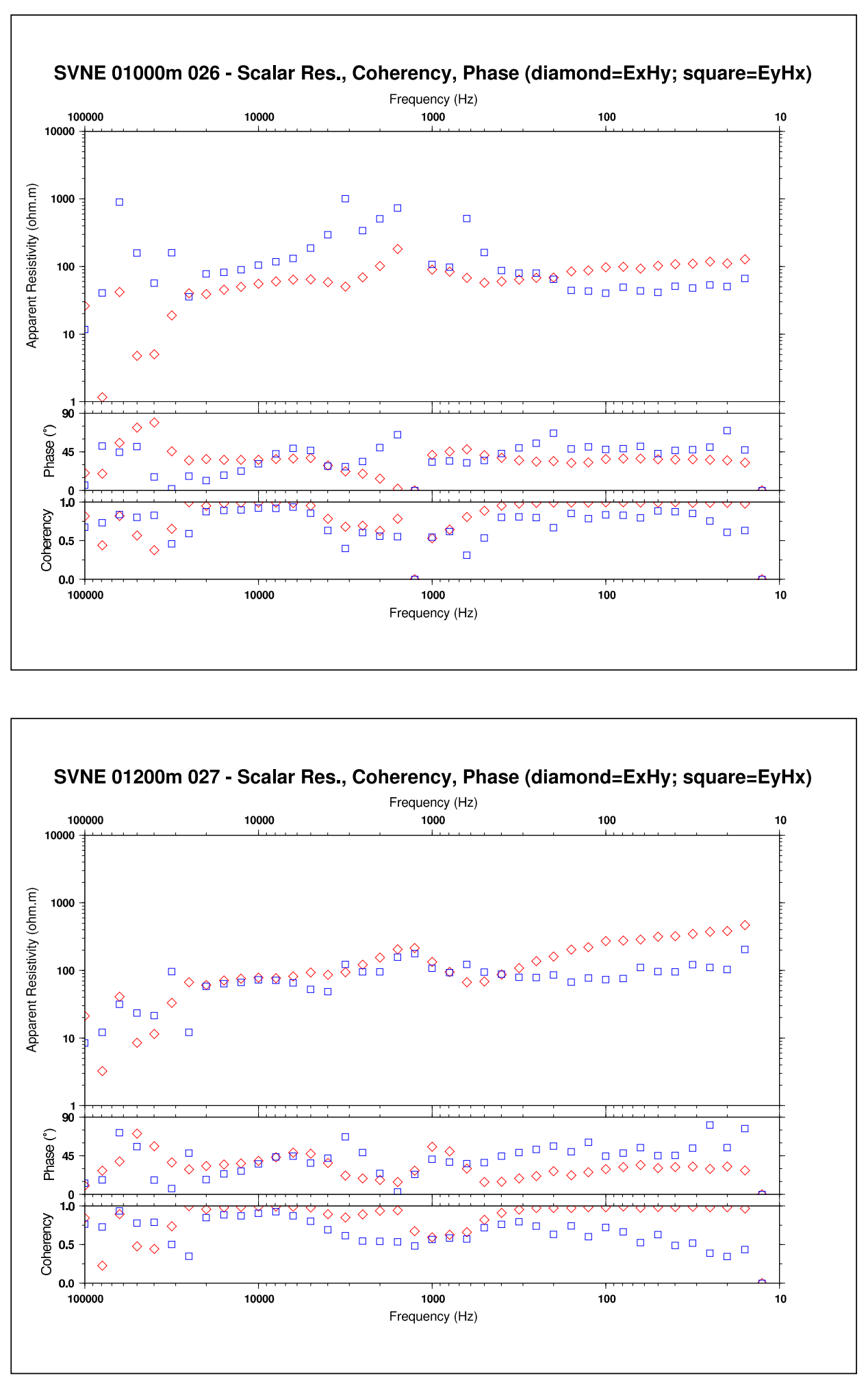